TUW-00-35

FSU-TPI 12/00

hep-th/0012219

\title{
General Two-Dimensional Supergravity from Poisson Superalgebras
}

\author{
M. Ertl*, W. Kummer ${ }^{\dagger}$, T. Strobl ${ }^{\ddagger}$, \\ ${ }^{* \dagger}$ Institut für Theoretische Physik \\ Technische Universität Wien \\ Wiedner Hauptstr. 8-10, A-1040 Wien, Austria \\ ${ }^{\ddagger}$ Institut für Theoretische Physik \\ Friedrich-Schiller-Universität Jena \\ Max Wien Platz 1, D-07743 Jena, Germany
}

\begin{abstract}
We provide the geometric actions for most general $N=1$ supergravity in two spacetime dimensions. Our construction implies an extension to arbitrary $N$. This provides a supersymmetrization of any generalized dilaton gravity theory or of any theory with an action being an (essentially) arbitrary function of curvature and torsion.

Technically we proceed as follows: The bosonic part of any of these theories may be characterized by a generically nonlinear Poisson bracket on a three-dimensional target space. In analogy to a given ordinary Lie algebra, we derive all possible $N=1$ extensions of any of the given Poisson (or $W$-) algebras. Using the concept of graded Poisson Sigma Models, any extension of the algebra yields a possible supergravity extension of the original theory, local Lorentz and super-diffeomorphism invariance follow by construction. Our procedure automatically restricts the fermionic extension to the minimal one; thus local supersymmetry is realized on-shell. By avoiding a superfield approach we are also able to circumvent in this way the introduction of constraints and their solution. For many well-known dilaton theories different supergravity extensions are derived. In generic cases their field equations are solved explicitly.
\end{abstract}

December 2000

\footnotetext{
*E-mail: ertl@tph.tuwien.ac.at

${ }^{\dagger}$ E-mail: wkummer@tph.tuwien.ac.at

${ }^{\ddagger}$ E-mail: Thomas.Strobl@tpi.uni-jena.de
} 


\section{Introduction}

The study of diffeomorphism invariant theories in $1+1$ dimensions for quite some time has been a fertile ground for acquiring some insight regarding the unsolved problems of quantum gravity in higher dimensions. Indeed, the whole field of spherically symmetric gravity belongs to this class, from $d$-dimensional Einstein theory to extended theories like the Jordan-Brans-Dicke theory [1-5] or 'quintessence' [6-11] which may now seem to obtain observational support [12-17]. Also equivalent formulations for 4d Einstein theory with nonvanishing torsion ('teleparallelism' [18-28]) and alternative theories including curvature and torsion [29] are receiving increasing attention.

On the other hand, supersymmetric extensions of gravity [30-33] are believed to be a necessary ingredient for a consistent solution of the problem to quantize gravity, especially within the framework of string/brane theory [34-36]. These extensions so far are based upon bosonic theories with vanishing torsion.

In view of this situation it seems surprising that the following problem so far has not been solved:

Given a general geometric action of pure gravity in two spacetime dimensions of the form (cf. [37-39] and references therein)

$$
L^{\mathrm{gr}}=\int d^{2} x \sqrt{-g} F\left(R, \tau^{2}\right),
$$

what are its possible supersymmetric generalizations?

Here $R$ and $\tau^{2}$ denote the in two dimensions only two algebraic-geometric invariants of curvature and torsion, respectively, and $F$ is some sufficiently wellbehaved function of them; for the case that $F$ does not depend on its second $\operatorname{argument,} R$ is understood to be the Ricci scalar of the torsion-free Levi-Civita connection.

As a prototype of a theory with dynamical torsion we may consider the specification of (1) to the Katanaev-Volovich (KV) model [40, 41], quadratic in curvature and torsion. Even for this relatively simple particular case of (1), a supergravity generalization has not been presented to this day.

The bosonic theory (1) may be reformulated as a first order gravity action (FOG) by introducing auxiliary fields $\phi$ and $X^{a}$ (the standard momenta in a Hamiltonian reformulation of the model; cf. [42, 43] for particular cases and [39] for the general discussion)

$$
L^{\mathrm{FOG}}=\int_{\mathcal{M}} \phi d \omega+X_{a} D e^{a}+\epsilon v(\phi, Y)
$$

where $Y=X^{2} / 2 \equiv X^{a} X_{a} / 2$ and $v$ is some two-argument function of the indicated variables. In (2) $e^{a}$ is the zweibein and $\omega_{a b}=\omega \epsilon_{a b}$ the Lorentz or spin connection,

both 1-form valued, and $\epsilon=\frac{1}{2} e^{a} \wedge e^{b} \epsilon_{b a}=e d^{2} x$ is the two-dimensional volume form $\left(e=\operatorname{det}\left(e_{m}{ }^{a}\right)\right)$. The torsion 2-form is $D e^{a}=d e^{a}+e^{b} \wedge \omega \epsilon_{b}{ }^{a}$. 
The function $v(\phi, Y)$ is the Legendre transform [39] of $F\left(R, \tau^{2}\right)$ with respect to the three arguments $R$ and $\tau^{a}$, or, if $F$ depends on the Levi-Civita curvature $R$ only, with respect to this single variable ( $v$ depending only on $\phi$ then). In view of its close relation to the corresponding quantity in generalized dilaton theories which we recall below, we shall call $\phi$ the 'dilaton' also within the action (2). ${ }^{1}$ The equivalence between (1) and the action (2) holds at a global level, if there is a globally well-defined Legendre transform of $F$. Prototypes are provided by quadratic actions, i.e. by $R^{2}$-gravity and the model of [40,41]. Otherwise generically the equivalence holds still locally (patchwise). Only theories for which $v$ or $F$ even locally do not have a Legendre transform, are not at all covered by the respective other formulation. In any case, as far as the supersymmetrization of $2 \mathrm{~d}$ gravity theories is concerned, we will henceforth focus on the family of actions given by (2).

The FOG formulation (2) also covers general dilaton theories in two dimensions $[44-51]$ ( $\widetilde{R}$ is the torsion free curvature scalar),

$$
L^{\mathrm{dil}}=\int d^{2} x \sqrt{-g}\left[\frac{\widetilde{R}}{2} \phi-\frac{1}{2} Z(\phi)\left(\partial^{n} \phi\right)\left(\partial_{n} \phi\right)+V(\phi)\right] .
$$

Indeed, by eliminating $X^{a}$ and the torsion-dependent part of $\omega$ in (2) by their algebraic equations of motion, for regular $2 \mathrm{~d}$ space-times $(e=\sqrt{-g} \neq 0)$ the theories (3) and (2) are locally and globally equivalent if in (2) the 'potential' is chosen as [52, 53] (cf. also Sec. 6.3 below for some details as well as [38] for a related approach)

$$
v^{\text {dil }}(\phi, Y)=Y Z(\phi)+V(\phi)
$$

There is also an alternative method for describing dilaton gravity by means of an action of the form (2), namely by using the variables $e^{a}$ as a zweibein for a metric $\bar{g}$, related to $g$ in (3) according to $g_{m n}=\Omega(\phi) \bar{g}_{m n}$ for a suitable choice of the function $\Omega$ (it is chosen in such a way that after transition from the Einstein-Cartan variables in (2) upon elimination of $X^{a}$ one is left with an action for $\bar{g}$ of the form (3) with $\bar{Z}=0$, i. e. without kinetic term for the dilaton, cf. e.g. $[44,46,54]){ }^{2}$ This formulation has the advantage that the resulting potential $v$ depends on $\phi$ only. It has to be noted, however, that due to a possibly singular behavior of $\Omega$ (or $1 / \Omega$ ) the global structures of the resulting spacetimes (maximally extended with respect to $\bar{g}$ versus $g$ ) are in part quite different. Moreover, also the change of variables in a path integral corresponding to the 'torsion' description of dilaton theories ( $Y$-dependent potential (4)) seems advantageous over the one in the 'conformal' description. In this description even

\footnotetext{
${ }^{1}$ In the literature also $\Phi=-\frac{1}{2} \ln \phi$ carries this name. This definition is useful when, as it is often the case for specific models, $\phi$ is restricted to $\mathbb{R}_{+}$only.

${ }^{2}$ Some details on the two approaches to general dilaton gravity may be found also in [39].
} 
interactions with (scalar) matter can be included in a systematic perturbation theory, starting from the (trivially) exact path integral for the geometric part (2) [55-57]. Therefore when describing dilaton theories within the present paper we will primarily focus on potentials (3), linear in $Y$.

In any case there is thus a huge number of $2 \mathrm{~d}$ gravity theories included in the present framework. We select only a few for illustrative purposes, one of which is spherically reduced Einstein gravity (SRG) from $d$ dimensions [58-61]

$$
Z_{\mathrm{SRG}}=-\frac{d-3}{(d-2) \phi}, \quad V_{\mathrm{SRG}}=-\lambda^{2} \phi^{\frac{d-4}{d-2}},
$$

where $\lambda$ is some constant; in the 'conformal approach' mentioned above the respective potentials become

$$
Z_{\overline{\mathrm{SRG}}}=0, \quad V_{\overline{\mathrm{SRG}}}=-\frac{\lambda^{2}}{\phi^{\frac{1}{d-2}}} .
$$

The KV-model, already referred to above, results upon

$$
Z_{\mathrm{KV}}=\alpha, \quad V_{\mathrm{KV}}=\frac{\beta}{2} \phi^{2}-\Lambda,
$$

where $\Lambda, \alpha$ and $\beta$ are constant. Two other particular examples are the so-called Jackiw-Teitelboim (JT) model [62-66] with vanishing torsion in (2) and no kinetic term of $\phi$ in (3),

$$
Z_{\mathrm{JT}}=0, \quad V_{\mathrm{JT}}=-\Lambda \phi,
$$

or the string inspired dilaton black hole (DBH) [67-69] (cf. also [70-78])

$$
Z_{\mathrm{DBH}}=-\frac{1}{\phi}, \quad V_{\mathrm{DBH}}=-\lambda^{2} \phi,
$$

which, incidentally, may also be interpreted as the formal limit $d \rightarrow \infty$ of (5).

For our present purposes it will be crucial that (2) may be formulated as a Poisson Sigma Model (PSM) [43, 48, 79, 80] (cf. also [81-85]). Collecting zero form and one-form fields within (2) as

$$
\left(X^{i}\right):=\left(\phi, X^{a}\right), \quad\left(A_{i}\right)=\left(d x^{m} A_{m i}(x)\right):=\left(\omega, e_{a}\right),
$$

and after a partial integration, the action (2) may be rewritten identically as

$$
L^{\mathrm{PSM}}=\int_{\mathcal{M}} d X^{i} \wedge A_{i}+\frac{1}{2} \mathcal{P}^{i j} A_{j} \wedge A_{i},
$$

where the matrix $\mathcal{P}^{i j}$ may be read off by direct comparison. The basic observation in this framework is that this matrix defines a Poisson bracket on the space 
spanned by coordinates $X^{i}$, which is then identified with the target space of a Sigma Model. In the present context this bracket $\left\{X^{i}, X^{j}\right\}:=\mathcal{P}^{i j}$ has the form

$$
\begin{aligned}
\left\{X^{a}, \phi\right\} & =X^{b} \epsilon_{b}{ }^{a}, \\
\left\{X^{a}, X^{b}\right\} & =v(\phi, Y) \epsilon^{a b},
\end{aligned}
$$

where (throughout this paper) $Y \equiv \frac{1}{2} X^{a} X_{a}$; this bracket may be verified to obey the Jacobi identity.

The gravitational origin of the underlying model is reflected by the first set of brackets: It shows that $\phi$ is the generator of Lorentz transformations (with respect to the bracket) on the target space $\mathbb{R}^{3}$. The form of the second set of brackets is already completely determined by this: Indeed, antisymmetry of the bracket leads to proportionality to the $\epsilon$-tensor, while the Jacobi identity for the bracket requires $v$ to be a function of the Lorentz invariant quantities $\phi$ and $X^{2}$ only.

Inspection of the local symmetries of a general PSM,

$$
\delta X^{i}=\mathcal{P}^{i j} \epsilon_{j}, \quad \delta A_{i}=-d \epsilon_{i}-\left(\partial_{i} \mathcal{P}^{j k}\right) \epsilon_{k} A_{j}
$$

shows that the Lorentz symmetry of the bracket gives rise to the local Lorentz symmetry of the gravity action (2) (specialization of (14) to an $\epsilon$ with only nonzero $\phi$ component, using the identification (10)). The second necessary ingredient for the construction of a gravity action, diffeomorphism invariance, on the other hand, is automatically respected by an action of the form (11). (It may be seen that the diffeomorphism invariance is also encoded on-shell by the remaining two local symmetries (14), cf. e.g. [48, 86]).

PSMs relevant for $2 \mathrm{~d}$ gravity theories (without further gauge field interactions) possess one 'Casimir function' $c(X)$ which is characterized by vanishing of the Poisson brackets $\left\{X^{i}, c\right\}$. Different constant values of $c$ characterize symplectic leaves $[87,88]$. In the language of gravity theories, for models with asymptotic Minkowski behavior $c$ is proportional to the ADM mass of the system. ${ }^{3}$

To summarize, the gravity models (11), and thus implicitly also any action of the form (2) and hence generically of (1), may be obtained from the construction of a Lorentz invariant bracket on the two-dimensional Minkowski space $\mathbb{R}^{2}$ spanned by $X^{a}$, with $\phi$ entering as an additional parameter. ${ }^{4}$ The resulting

\footnotetext{
${ }^{3}$ We remark in parenthesis that an analogous conservation law may be established also in the presence of additional matter fields [89, 90].

${ }^{4}$ Actually, this point of view was already used in [43] so as to arrive at (11), without, however, fully realizing the relation to (1) at that time. Let us remark on this occasion that in principle one might also consider theories (11) with $X^{a}$ replaced e. g. by $X^{a} \cdot f\left(\phi, X^{2}\right)$. For a nonvanishing function $f$ after a suitable reparametrization of the target space this again yields a PSM. Also the identification of the gauge fields $A_{i}$ in (10) could be modified in a similar manner. Hence we do not have to cover this possibility explicitly in what follows. Nevertheless, it could be advantageous to derive by this means a more complicated gravity model from a simpler PSM structure.
} 
bracket as well as the corresponding models are seen to be parametrized by one two-argument function $v$ in this way.

The Einstein-Cartan formulation of $2 \mathrm{~d}$ gravities as in (2) or, even more so, in the PSM form (11) will turn out to be particularly convenient for obtaining the most general supergravities in $d=2$. Whereas the metrical formulation of gravity due to Einstein in $d=4$ appeared very cumbersome for a supersymmetric generalization, the Einstein-Cartan approach appeared to be best suited for the needs of introducing additional fermionic degrees of freedom to pure gravity [30$33]$.

We now briefly digress to the corresponding strategy of constructing a supersymmetric extension of a gravity theory in a spacetime of general dimension $d$. By adding to vielbein $e_{m}{ }^{a}$ and Lorentz connection $\omega_{m a}{ }^{b}$ appropriate terms containing a fermionic and spinor valued 1-form $\psi_{m}{ }^{\alpha}$, the Rarita-Schwinger field, an action invariant under local supersymmetry can be constructed, where $\psi_{m}{ }^{\alpha}$ plays the role of the gauge field for that symmetry. In this formulation the generic local infinitesimal supersymmetry transformations are of the form

$$
\delta e_{m}^{a}=-2 i\left(\epsilon \gamma^{a} \psi_{m}\right), \quad \delta \psi_{m}^{\alpha}=-D_{m} \epsilon^{\alpha}+\cdots
$$

with $\epsilon=\epsilon(x)$ arbitrary.

In the course of time various methods were developed to make the construction of supergravity actions more systematical. One of these approaches, relying on superfields [91-95], extends the Einstein-Cartan formalism by adding anticommuting coordinates to the space-time manifold, thus making it a supermanifold, and, simultaneously, by enlarging the structure group with a spinorial representation of the Lorentz group. This method adds a huge number of unphysical fields and unwanted symmetries to the theory, which can be eliminated by choosing appropriate constraints on supertorsion and supercurvature and by choosing a Wess-Zumino type gauge. The need for constraints and their consistent embedding into the Bianchi identities is the draw-back of this method, because the argumentation for a particular set of them is mainly of technical nature and quite involved.

The other systematic approach to construct supergravity models for general $d$ is based on the similarity of gravity to a gauge theory. The (inverse) vielbein and the Lorentz-connection are treated as gauge fields on a similar footing as gauge fields of possibly additional gauge groups. Curvature and torsion appear as particular components of the total field strength. ${ }^{5}$ By adding fermionic symmetries to the gravity gauge group, usually taken as the Poincaré, de Sitter, or conformal group, one obtains the corresponding supergravity theories [97].

\footnotetext{
${ }^{5}$ Note that nevertheless standard gravity theories cannot be just reformulated as YM gauge theories with all symmetries being incorporated in a principle fiber bundle description; there still is the infinite dimensional diffeomorphism group one has to deal with (cf. also [96] for an illustration).
} 
In the two-dimensional case, the supergravity multiplet was first constructed using the superfield approach [98]. Based on that formalism, it was straightforward to formulate a supersymmetric generalization of the dilaton theory (3), cf. [99]. Before that the supersymmetric generalization of the particular case of the Jackiw-Teitelboim or de Sitter model [62-66] has been achieved within this framework in [100]. Up to global issues, this solved implicitly also the problem of a supersymmetrization of the theories (1) in the torsion-free case.

Still, the supergravity multiplet obtained from the set of constraints used in [98] consists of the vielbein, the Rarita-Schwinger field and an auxiliary scalar field, but the Lorentz-connection is lost as independent field. It is expressed in terms of vielbein and Rarita-Schwinger field. Without a formalism using an independent Lorentz-connection the construction of supersymmetric versions of general theories of the $F\left(R, \tau^{2}\right)$-type is impossible. Although there was some partial success in constructing supergravity models of such theories by relaxing the constraints of Howe, the result turned out to be too clumsy to allow further development so far [101].

As in higher dimensional theories, the gauge theoretic approach provides a much simpler method for supersymmetrization than the superfield approach. However, it is restricted to relatively simple Lagrangians such as the one of the Jackiw-Teitelboim model (8) [102, 103]. The generic model (2) or also (3) cannot be treated in this fashion.

On the other hand, first attempts showed that super dilaton theories may fit into the framework of 'nonlinear' supergauge theories [104, 105], and the action for a super dilaton theory was obtained (without superfields) by a nonlinear deformation of the graded de Sitter group using free differential algebras in [106].

Recently, it turned out [107] (but cf. also [104, 105]) that the framework of PSMs $[43,79,80]$, now with a graded target space, represents the formalism of choice to deal with super dilaton theories. In particular, it allowed for a simple derivation of the general solution of the corresponding field equations, and in this process yielded the somewhat surprising result that, in the absence of additional matter fields, the supersymmetrization of the dilaton theories (3) is on-shell trivial. By this we mean that, up to the choice of a gauge, in the general solution to the field equations all fermionic fields can be made to vanish identically by an appropriate choice of gauge while the bosonic fields satisfy the field equations of the purely bosonic theory and are still subject to the symmetries of the latter. This local on-shell triviality of the supersymmetric extension may be interpreted superficially to be yet another consequence of the fact that, from the Hamiltonian point of view, the 'dynamics' of (11) is described by just one variable (the Casimir function) which does not change when fermionic fields are added. This type of triviality will cease to prevail in the case of additional matter fields (as is already obvious from a simple counting of fields and local symmetries involved). The supersymmetrization may be used [99], furthermore, as a technical device to prove positive energy theorems for supersymmetric and non-supersymmetric dilaton 
theories. Thus, the (local) on-shell triviality of pure $2 \mathrm{~d}$ supergravity theories by no means implicitly demolishes all the possible interest in their supersymmetric generalizations. This applies similarly to the $F\left(R, \tau^{2}\right)$-theories and to the FOG formulation (2), we are primarily interested in within the present paper.

Graded PSMs (gPSMs) turn out to also provide a unifying and most efficient framework for the construction of supersymmetric extensions of a twodimensional gravity theory, at least as far as theories of the initially mentioned type (1) are considered. This route, sketched already briefly in [107], will be followed in detail within the present paper.

The main idea of this approach will be outlined in Sec. 2.1. It will be seen that within this framework the problem for a supersymmetric extension of a gravity theory (1) is reduced to a finite dimensional problem: Given a Lorentz invariant Poisson bracket on a two-dimensional Minkowski space (which in addition depends also on the 'dilaton' or, equivalently, on the generator of Lorentz transformations $\phi$ ), one has to extend this bracket consistently and in a Lorentz covariant manner to the corresponding superspace.

In spirit this is closely related to the analogous extension of Lie algebras to superalgebras [108-111]. In fact, in the particular case of a linear dependence of $v$ in (13) the original Poisson bracket corresponds to a three-dimensional Lie algebra, and likewise any linear extension of this Poisson algebra to a superalgebra. Here we are dealing with general nonlinear Poisson algebras, particular cases of which can be interpreted as finite $W$-algebras (cf. [112]). Due to that nonlinearity the analysis necessary for the fermionic extension is much more involved and there is a much higher ambiguity in an extension (except if one considers this only modulo arbitrary (super)diffeomorphisms). For this reason we mainly focus on an $N=1$ extension within this paper.

After recapitulating some material on gPSMs in Sec. 2.2, setting also our notation and conventions, the solution of the $\phi$-components of the Jacobi identities is given in Sec. 3.1 simply by writing down the most general Lorentz covariant ansatz for the Poisson tensor. In Sec. 3.2 the remaining Jacobi identities are solved in full generality for nondegenerate and degenerate $N=1$ fermionic extension.

The observation that a large degree of arbitrariness is present in these extensions is underlined also by the study of target space diffeomorphism in Sec. 4 . We also point out the advantages of this method in the quest for new algebras and corresponding gravity theories.

In Sec. 5 we shall consider particular examples of the general result. This turns out to be much superior than to perform a general abstract discussion of the results of Sec. 3. The more so, because fermionic extensions of specific bosonic $2 \mathrm{~d}$ gravity theories can be investigated which have been discussed already in the literature. Supersymmetric extensions of the KV-model (7) as compared to SRG (5) will serve for illustrative purposes.

The corresponding actions and their relation to the initial problem (1) are 
given in Sec. 7. Also the general relation to the supersymmetric dilatonic theories (3) will be made explicit using the results of Sec. 6. Several different supersymmetrizations (one of which is even parity violating) for the example of SRG are compared to the one provided previously in the literature [99]. For each model the corresponding supersymmetry is given explicitly.

In Sec. 8 the explicit solution for a supergravity theory with the bosonic part corresponding to $v^{\text {dil }}$ in (4) is given.

In the final Sec. 9 we will summarize our findings and comment on possible further investigations.

App. A defines notations and summarizes some useful identities.

\section{Graded Poisson Sigma Model}

\subsection{Outline of the approach}

The PSM formulation of gravity theories allows a direct generalization, yielding possible supergravity theories. Indeed, from this perspective it is suggestive to replace the Minkowski space with its linear coordinates $X^{a}$ by its superspace analogue, spanned by $X^{a}$ and (real, i. e. Majorana) spinorial and (one or more) Grassmann-valued coordinates $\chi^{i \alpha}$ (where $\left.i=1, \ldots, N\right)$. In the purely bosonic case we required that $\phi$ generates Lorentz transformations on Minkowski space. We now extend this so that $\phi$ is the generator of Lorentz transformations on superspace. This implies in particular that beside (12) now also

$$
\left\{\chi^{i \alpha}, \phi\right\}=-\frac{1}{2} \chi^{i \beta}\left(\gamma^{3}\right)_{\beta}^{\alpha}
$$

has to hold, where $-\frac{1}{2}\left(\gamma^{3}\right)_{\beta}{ }^{\alpha}$ is the generator of Lorentz transformations in the spinorial representation. For the choice of the $\gamma$-matrices and further details on notation and suitable identities we refer to App. A.

Within the present work we first focus merely on a consistent extension of the original bosonic Poisson algebra to the total superspace. This superspace can be built upon $N$ pairs of coordinates obeying (16). Given such a graded Poisson algebra, the corresponding Sigma Model provides a possible $N$-supergravity extension of the original gravity model corresponding to the purely bosonic sigma model. We shall mainly focus on the construction of a graded Poisson tensor $\mathcal{P}^{I J}$ for the simplest supersymmetric extension $N=1$, i. e. on a ('warped') product of the above superspace and the linear space spanned by the generator $\phi$. Upon restriction to the bosonic submanifold $\chi^{\alpha}=0$, the bracket will be required to coincide with the bracket (12) and (13) corresponding to the bosonic theory (11). Just as the framework of PSMs turns out to provide a fully satisfactory and consistent $2 d$ gravity theory with all the essential symmetries for any given (Lorentz invariant) Poisson bracket (12) and (13), the framework of graded Poisson Sigma 
Models (gPSM) will provide possible generalizations for any of the brackets $\mathcal{P}^{i j}$ with a local 'supersymmetry' of the generic type (15). In particular, by construction of the general theory (cf [107] or Sec. 3 below) and upon an identification which is a straightforward extension of (10), the resulting gravity theory will be invariant automatically with respect to local Lorentz transformations, spacetime diffeomorphisms and local supersymmetry transformations. In particular, the Rarita-Schwinger field $\psi_{\alpha}$ (or $\psi_{\mathfrak{i} \alpha}, \mathfrak{i}=1, \ldots, N$ in the more general case) is seen to enter naturally as the fermionic component of the one-form valued multiplet $A_{I}$. Likewise, specializing the local symmetries (14) (or rather their generalization to the graded case provided in (30) below) to the spinorial part $\epsilon_{\alpha}$, local supersymmetry transformations of the form (15) are found, which, by construction, are symmetries of the action. (In fact, it is here where the graded Jacobi identity for $\mathcal{P}$ enters as an essential ingredient!) Finally, by construction, the bosonic part of the action of the gPSM corresponding to the bracket $\mathcal{P}^{I J}$ will coincide with (11). Thus, for any such a bracket $\mathcal{P}^{I J}$, the resulting model should allow the interpretation as a permissible supersymmetric generalization of the original bosonic starting point.

The relations (12), (16) fix the $\phi$ components of the sought for (graded) Poisson tensor $\mathcal{P}^{I J}$. We are thus left with determining the remaining components $\mathcal{P}^{A B}, A$ and $B$ being indices in the four-dimensional superspace with $X^{A}=\left(X^{a}, \chi^{\alpha}\right)$. As will be recapitulated in Sec. 2.2, beside the graded symmetry of the tensor $\mathcal{P}^{I J}$, the only other requirement it has to fulfil by definition is the graded Jacobi identity. This is of the form of the vanishing of a 3 -tensor $J^{I J K}$ (cf. (19) below), which may be expressed also as the Schouten-Nijenhuis bracket $[\cdot, \cdot]_{S N}$ of $\left(\mathcal{P}^{I J}\right)$ with itself. In this formulation $\left(\mathcal{P}^{I J}\right)$ is meant to be the Poisson tensor itself and not its components (abstract indices). It is straightforward to verify (cf. also [107]) that the relations $J^{I J K}=0$ with at least one of the indices coinciding with the one corresponding to $\phi$ are satisfied, iff $\left(\mathcal{P}^{A B}\right)$ is a Lorentz covariant 2-tensor,

$$
\mathcal{L}_{\left(\mathcal{P}^{A \phi}\right)}\left(\mathcal{P}^{A B}\right)=0
$$

i. e. depending on $X^{a}, \chi^{\alpha}$ and also on the Lorentz invariant quantity $\phi$ in a covariant way as determined by its indices. Thus one is left with finding the general solution of $J^{A B C}=0$ starting from a Lorentz covariant ansatz for $\left(\mathcal{P}^{A B}\right)$.

Let us note on this occasion that the above considerations do not imply that $\left(\mathcal{P}^{A B}\right)$ forms a bracket on the Super-Minkowski space, a subspace of the target space under discussion. The reason is that the equations $J^{A B C}=0$ contain also derivatives of $\mathcal{P}^{A B}$ with respect to $\phi$ : in terms of the Schouten-Nijenhuis bracket, the remaining equations become

$$
\left[\left(\mathcal{P}^{A B}\right),\left(\mathcal{P}^{A B}\right)\right]_{S N}=\left(\mathcal{P}^{A \phi}\right) \wedge\left(\partial_{\phi} \mathcal{P}^{A B}\right),
$$

where the components of the supervector $\left(\mathcal{P}^{A \phi}\right)$ are given implicitly by eqs. (12) and (16) above. So $\left(\mathcal{P}^{A B}\right)$ defines a graded Poisson bracket for the $X^{A}$ only if 
it is independent of $\phi$. However, in the present context $\phi$-independent Poisson tensors are uninteresting in view of our discussion of actions of the form (2).

It should be remarked that given a particular bosonic model and its corresponding bracket, there is by no means a unique graded extension, even for fixed $N$. Clearly, any (super-)diffeomorphism leaving invariant the bosonic sector as well as the brackets (16) applied to a solution of the (graded) Jacobi identities yields another solution. This induces an ambiguity for the choice of a superextension of a given gravity model (3) or also (1). This is in contrast to the direct application of, say, the superfield formalism of Howe [98], which when applied to the (necessarily torsionfree) theory (3) [99], yields one particular superextension. This now turns out as just one possible choice within an infinite dimensional space of admissible extensions. From one or the other perspective, however, different extensions (for a given $N$ ) may be regarded also as effectively equivalent. We shall come back to these issues below.

A final observation concerns the relation of our supersymmetric extensions to 'ordinary' supergravity. From the point of view of the seminal work on the $2 \mathrm{~d}$ analogue [33, 113] of 4d supergravity our supergravity algebra is 'deformed' by the presence of a dilaton field. Such a feature is known also from the dimensional reduction of supergravity theories in higher dimensions, where one or more dilaton fields arise from the compactification.

\subsection{Details of the gPSM}

In this Section we recollect for completeness some general and elementary facts about graded Poisson brackets and the corresponding Sigma Models. This Section (cf. also App. A) also sets the conventions about signs etc. used within the present paper, which are adapted to those of [101] and which differ on various instances from those used in [107].

For the construction of the gPSM we take a 2-dimensional base manifold $\mathcal{M}$, also called world sheet or space-time manifold, with purely bosonic (commutative) coordinates $x^{m}$, and the target space $\mathcal{N}$ with coordinates $X^{I}=\left(\phi, X^{A}\right)=$ $\left(\phi, X^{a}, \chi^{\alpha}\right), \phi$ and $X^{a}$ being bosonic and $\chi^{\alpha}$ fermionic (anticommutative), promoting $\mathcal{N}$ to a supermanifold. The restriction to one Majorana spinor means that only the case $N=1$ is implied in what follows. To the coordinate functions $X^{I}$ correspond gauge fields $A_{I}$ which we identify with the usual Lorentzconnection 1-form $\omega$ and the vielbein 1-form $e_{a}$ of the Einstein-Cartan formalism of gravity and with the Rarita-Schwinger 1-form $\psi_{\alpha}$ of supergravity according to $A_{I}=\left(\omega, e_{A}\right)=\left(\omega, e_{a}, \psi_{\alpha}\right)$. They can be viewed as 1 -forms on the base manifold $\mathcal{M}$ with values in the cotangential space of $\mathcal{N}$, and may be collected in the total 1-1-form $A=d X^{I} A_{I}=d X^{I} d x^{m} A_{m I}$. As the main structure of the model we choose a Poisson tensor $\mathcal{P}^{I J}=\mathcal{P}^{I J}(X)$ on $\mathcal{N}$, which encodes the desired symmetries and the dynamics of the theory to be constructed. Due to the grading of the coordinates of $\mathcal{N}$ it is graded antisymmetric $\mathcal{P}^{I J}=-(-1)^{I J} \mathcal{P}^{J I}$ and is 
assumed to fulfil the graded Jacobi identity $\left(\vec{\partial}_{I}=\vec{\partial} / \partial X^{I}\right.$ is the right derivative, cf. App. A) of which we list also a convenient alternative version

$$
\begin{aligned}
J^{I J K} & =\mathcal{P}^{I L} \vec{\partial}_{L} \mathcal{P}^{J K}+\operatorname{gcycl}(I J K) \\
& =\mathcal{P}^{I L} \vec{\partial}_{L} \mathcal{P}^{J K}+\mathcal{P}^{J L} \vec{\partial}_{L} \mathcal{P}^{K I}(-1)^{I(J+K)}+\mathcal{P}^{K L} \vec{\partial}_{L} \mathcal{P}^{I J}(-1)^{K(I+J)} \\
& =3 \mathcal{P}^{I] L} \vec{\partial}_{L} \mathcal{P}^{[J K}=0 .
\end{aligned}
$$

The Poisson tensor defines the Poisson bracket of functions $f, g$ on $\mathcal{N}$,

$$
\{f, g\}=\left(f \overleftarrow{\partial}_{J}\right) \mathcal{P}^{J I}\left(\vec{\partial}_{I} g\right)
$$

implying for the coordinate functions $\left\{X^{I}, X^{J}\right\}=\mathcal{P}^{I J}$. With (323) the Poisson bracket (22) may be written also as

$$
\{f, g\}=\mathcal{P}^{J I}\left(\vec{\partial}_{I} g\right)\left(\vec{\partial}_{J} f\right)(-1)^{g(f+J)} .
$$

This bracket is graded anticommutative,

$$
\{f, g\}=-(-1)^{f g}\{g, f\}
$$

and fulfils the graded Jacobi identity

$$
\left\{X^{I},\left\{X^{J}, X^{K}\right\}\right\}(-1)^{I K}+\left\{X^{J},\left\{X^{K}, X^{I}\right\}\right\}(-1)^{J I}+\left\{X^{K},\left\{X^{I}, X^{J}\right\}\right\}(-1)^{K J}=0,
$$

which is equivalent to the graded derivation property

$$
\left\{X^{I},\left\{X^{J}, X^{K}\right\}\right\}=\left\{\left\{X^{I}, X^{J}\right\}, X^{K}\right\}+(-1)^{I J}\left\{X^{J},\left\{X^{I}, X^{K}\right\}\right\} .
$$

The PSM action (11) generalizes to

$$
L^{\mathrm{gPSM}}=\int_{\mathcal{M}} d X^{I} A_{I}+\frac{1}{2} \mathcal{P}^{I J} A_{J} A_{I},
$$

where in the graded case the sequence of the indices is important. The functions $X^{I}(x)$ represent a map from the base manifold to the target space in the chosen coordinate systems of $\mathcal{M}$ and $\mathcal{N}$, and $d X^{I}$ is the shorthand notation for the derivatives $d^{\mathcal{M}} X^{I}(x)=d x^{m} \partial_{m} X^{I}(x)$ of these functions. The reader may notice the overloading of the symbols $X^{I}$ which sometimes are used to denote the map from the base manifold to the target space and sometimes, as in the paragraph above, stand for target space coordinates. This carries over to other expressions like $d X^{I}$ which denote the coordinate differentials $d^{\mathcal{N}} X^{I}$ on $\mathcal{N}$ and, on other occasions, as in the action (27), the derivative of the map from $\mathcal{M}$ to $\mathcal{N}$. 
The variation of $A_{I}$ and $X^{I}$ in (27) yields the gPSM field equations

$$
\begin{gathered}
d X^{I}+\mathcal{P}^{I J} A_{J}=0, \\
d A_{I}+\frac{1}{2}\left(\vec{\partial}_{I} \mathcal{P}^{J K}\right) A_{K} A_{J}=0 .
\end{gathered}
$$

These are first order differential equations of the fields $X^{I}(x)$ and $A_{m I}(x)$ and the Jacobi identity (19) of the Poisson tensor ensures the closure of (28) and (29). As a consequence of (19) the action exhibits the symmetries

$$
\delta X^{I}=\mathcal{P}^{I J} \epsilon_{J}, \quad \delta A_{I}=-d \epsilon_{I}-\left(\vec{\partial}_{I} \mathcal{P}^{J K}\right) \epsilon_{K} A_{J},
$$

where corresponding to each gauge field $A_{I}$ we have a symmetry parameter $\epsilon_{I}(x)$ with the same grading which is a function of $x$ only. When calculating the commutator of these symmetries, in general parameters depending on both $x$ and $X$ are obtained. For two parameters $\epsilon_{1 I}(x, X)$ and $\epsilon_{2 I}(x, X)$

$$
\begin{aligned}
& \left(\delta_{1} \delta_{2}-\delta_{2} \delta_{1}\right) X^{I}=\delta_{3} X^{I} \\
& \left(\delta_{1} \delta_{2}-\delta_{2} \delta_{1}\right) A_{I}=\delta_{3} A_{I}+\left(d X^{J}+\mathcal{P}^{J K} A_{K}\right)\left(\vec{\partial}_{J} \vec{\partial}_{I} \mathcal{P}^{R S}\right) \epsilon_{1 S} \epsilon_{2 R}
\end{aligned}
$$

follows, where $\epsilon_{3 I}(x, X)$ of the resulting variation $\delta_{3}$ are given by the Poisson bracket (or Koszul-Lie bracket) of the 1-forms $\epsilon_{1}=d X^{I} \epsilon_{1 I}$ and $\epsilon_{2}=d X^{I} \epsilon_{2 I}$, defined according to

$$
\epsilon_{3 I}=\left\{\epsilon_{2}, \epsilon_{1}\right\}_{I}:=\left(\vec{\partial}_{I} \mathcal{P}^{J K}\right) \epsilon_{1 K} \epsilon_{2 J}+\mathcal{P}^{J K}\left(\epsilon_{1 K} \vec{\partial}_{J} \epsilon_{2 I}-\epsilon_{2 K} \vec{\partial}_{J} \epsilon_{1 I}\right) .
$$

Note, that the commutator of the PSM symmetries closes if the Poisson tensor is linear, for non-linear Poisson tensors the algebra closes only on-shell (32).

Right and left Hamiltonian vector fields are defined by $\vec{T}^{I}=\left\{X^{I}, \cdot\right\}$ and $\overleftarrow{T}^{I}=\left\{\cdot, X^{I}\right\}$, respectively, i. e. by

$$
\vec{T}^{I} \cdot f=\left\{X^{I}, f\right\}=\mathcal{P}^{I J}\left(\vec{\partial}_{J} f\right), \quad f \cdot \overleftarrow{T}^{I}=\left\{f, X^{I}\right\}=\left(f \overleftarrow{\partial}_{J}\right) \mathcal{P}^{J I}
$$

The vector fields $\overleftarrow{T}^{I}$ are the generators of the symmetries, $\delta X^{I}=X^{I} \cdot \overleftarrow{T}^{J} \epsilon_{J}$. From their commutator the algebra

$$
\left[\overleftarrow{T}^{I}, \overleftarrow{T}^{J}\right]=\overleftarrow{T}^{K} f_{K}{ }^{I J}(X)
$$

follows with the structure functions $f_{K}^{I J}=\left(\vec{\partial}_{K} \mathcal{P}^{I J}\right)$. Structure constants and therefore Lie algebras are obtained when the Poisson tensor depends only linearly on the coordinates, which is true for Yang-Mills gauge theory and simple gravity models like (anti-)de Sitter gravity.

As in the purely bosonic case the kernel of the graded Poisson algebra determines the so-called Casimir functions $C$ obeying $\left\{C, X^{I}\right\}=0$. When the co-rank 
of the bosonic theory - with one Casimir function - is not changed we shall call this case non-degenerate. Then $\mathcal{P}^{\alpha \beta}$, the bosonic part of the fermionic extension, must be of full rank. For $N=1$ supergravity and thus one target space Majorana spinor $\chi^{\alpha}$, the expansion of $C$ in $\chi^{\alpha}$ reads $\left(\chi^{2}=\chi^{\alpha} \chi_{\alpha}\right.$, cf. App. A)

$$
C=c+\frac{1}{2} \chi^{2} c_{2},
$$

where $c$ and $c_{2}$ are functions of $\phi$ and $Y \equiv \frac{1}{2} X^{a} X_{a}$ only. This assures that the Poisson bracket $\{\phi, C\}$ is zero. From the bracket $\left\{X^{a}, C\right\}=0$, to zeroth order in $\chi^{\alpha}$, the defining equation of the Casimir function for pure bosonic gravity PSMs becomes

$$
\nabla c:=\left(\partial_{\phi}-v \partial_{Y}\right) c=0 .
$$

This is the well-known partial differential equation of that quantity [39, 48]. The solution of (37) for bosonic potentials relevant for kinetic dilaton theories (4) can be given by ordinary integration,

$$
\begin{aligned}
c(\phi, Y) & =Y e^{Q(\phi)}+W(\phi), \\
Q(\phi) & =\int_{\phi_{1}}^{\phi} Z(\varphi) d \varphi, \quad W(\phi)=\int_{\phi_{0}}^{\phi} e^{Q(\varphi)} V(\varphi) d \varphi .
\end{aligned}
$$

The new component $c_{2}$ is derived by considering the terms proportional to $\chi^{\beta}$ in the bracket $\left\{\chi^{\alpha}, C\right\}=0$. Thus $c_{2}$ will depend on the specific fermionic extension. In the degenerate case, when $\mathcal{P}^{\alpha \beta}$ is not of full rank, there will be more than one Casimir function, including purely Grassmann valued ones (see Sec. 3.2.2 and 3.2.3).

\section{Solution of the Jacobi-Identities}

As mentioned above, in order to obtain the general solution of the graded Jacobi identities a suitable starting point is the use of Lorentz symmetry in a most general ansatz for $\mathcal{P}^{I J}$. Alternatively, one could use a simple $\mathcal{P}_{(0)}^{I J}$ which trivially fulfils (19). Then the most general $\mathcal{P}^{I J}$ may be obtained by a general diffeomorphism in target space. The first route will be followed within this section. We will comment upon the second one in Sec. 4.

\subsection{Lorentz-Covariant Ansatz for the Poisson-Tensor}

Lorentz symmetry determines the mixed components $\mathcal{P}^{A \phi}$ of $\mathcal{P}^{I J}$,

$$
\mathcal{P}^{a \phi}=X^{b} \epsilon_{b}{ }^{a}, \quad \mathcal{P}^{\alpha \phi}=-\frac{1}{2} \chi^{\beta}\left(\gamma^{3}\right)_{\beta}{ }^{\alpha} .
$$


All other components of the Poisson tensor must be Lorentz-covariant (cf. the discussion around (17)). Expanding them in terms of invariant tensors $\eta^{a b}, \epsilon^{a b}$, $\epsilon^{\alpha \beta}$ and $\gamma$-matrices yields

$$
\begin{aligned}
\mathcal{P}^{a b} & =V \epsilon^{a b}, \\
\mathcal{P}^{\alpha b} & =\chi^{\beta}\left(F^{b}\right)_{\beta}{ }^{\alpha}, \\
\mathcal{P}^{\alpha \beta} & =U\left(\gamma^{3}\right)^{\alpha \beta}+i \widetilde{U} X^{c}\left(\gamma_{c}\right)^{\alpha \beta}+i \widehat{U} X^{c} \epsilon_{c}{ }^{d}\left(\gamma_{d}\right)^{\alpha \beta} .
\end{aligned}
$$

The quantities $V, U, \widetilde{U}$ and $\widehat{U}$ are functions of $\phi, Y$ and $\chi^{2}$. Due to the anticommutativity of $\chi^{\alpha}$ the dependence on $\chi^{2}$ is at most linear. Therefore

$$
V=v(\phi, Y)+\frac{1}{2} \chi^{2} v_{2}(\phi, Y)
$$

depends on two Lorentz-invariant functions $v$ and $v_{2}$ of $\phi$ and $Y$. An analogous notation will be implied for $U, \widetilde{U}$ and $\widehat{U}$, using the respective small case letter for the $\chi$-independent component of the superfield and an additional index 2 for the respective $\chi^{2}$-component. The component (42) contains the spinor matrix $\left(F^{a}\right)_{\beta}{ }^{\gamma}$, which may be first expanded in terms of the linearly independent $\gamma$-matrices,

$$
\left(F^{a}\right)_{\beta}^{\gamma}=f_{(1)}^{a} \delta_{\beta}^{\gamma}+i f^{a b}\left(\gamma_{b}\right)_{\beta}^{\gamma}+f_{(5)}^{a}\left(\gamma^{3}\right)_{\beta}{ }^{\gamma} .
$$

The Lorentz-covariant coefficient functions in (45) are further decomposed according to

$$
\begin{aligned}
& f_{(1)}^{a}=f_{(11)} X^{a}-f_{(12)} X^{b} \epsilon_{b}{ }^{a}, \\
& f_{(5)}^{a}=f_{(51)} X^{a}-f_{(52)} X^{b} \epsilon_{b}{ }^{a}, \\
& f^{a b}=f_{(s)} \eta^{a b}+f_{(t)} X^{a} X^{b}-f_{(h)} X^{c} \epsilon_{c}{ }^{a} X^{b}+f_{(a)} \epsilon^{a b} .
\end{aligned}
$$

The eight Lorentz-invariant coefficients $f_{(11)}, f_{(12)}, f_{(51)}, f_{(52)}, f_{(s)}, f_{(t)}, f_{(h)}$ and $f_{(a)}$ are functions of $\phi$ and $Y$ only. The linearity in $\chi^{\alpha}$ of (42) precludes any $\chi^{2}$ term in (45).

Below it will turn out to be convenient to use a combined notation for the bosonic and the $\chi^{2}$-dependent part of $\mathcal{P}^{\alpha \beta}$,

$$
\mathcal{P}^{\alpha \beta}=v^{\alpha \beta}+\frac{1}{2} \chi^{2} v_{2}^{\alpha \beta},
$$

where $v^{\alpha \beta}$ and $v_{2}^{\alpha \beta}$ are particular matrix-valued functions of $\phi$ and $X^{a}$, namely, in the notation above (cf. also App. A for the definition of $X^{++}$and $X^{--}$),

$$
v^{\alpha \beta}=\left(\begin{array}{cc}
\sqrt{2} X^{++}(\tilde{u}-\hat{u}) & -u \\
-u & \sqrt{2} X^{--}(\tilde{u}+\hat{u})
\end{array}\right),
$$

and likewise with suffix 2 . Note that the symmetric $2 \times 2$ matrix $v^{\alpha \beta}$ still depends on three arbitrary real functions; as a consequence of Lorentz invariance, however, they are functions of $\phi$ and $Y$ only. A similar explicit matrix representation may be given also for $F_{\alpha}^{ \pm \pm}$. 


\subsection{Remaining Jacobi Identities}

The Jacobi identities $J^{\phi B C}=0$ have been taken care of automatically by the Lorentz covariant parametrization introduced in Sec. 3.1. In terms of these functions we write the remaining identities as

$$
\begin{aligned}
J^{\alpha \beta \gamma} & =\vec{T}^{\alpha}\left(\mathcal{P}^{\beta \gamma}\right)+\operatorname{cycl}(\alpha \beta \gamma)=0, \\
J^{\alpha \beta c} & =\vec{T}^{c}\left(\mathcal{P}^{\alpha \beta}\right)+\vec{T}^{\alpha}\left(\chi F^{c}\right)^{\beta}+\vec{T}^{\beta}\left(\chi F^{c}\right)^{\alpha}=0, \\
\frac{1}{2} J^{\alpha b c} \epsilon_{c b} & =\vec{T}^{\alpha}(V)-\vec{T}^{b}\left(\chi F^{c}\right)^{\alpha} \epsilon_{c b}=0 .
\end{aligned}
$$

Here $\vec{T}^{a}$ and $\vec{T}^{\alpha}$ are Hamiltonian vector fields introduced in (34), yielding $\left(\partial_{\phi}=\right.$ $\left.\frac{\partial}{\partial \phi}, \partial_{a}=\frac{\partial}{\partial X^{a}}, \partial_{\alpha}=\frac{\partial}{\partial \chi^{\alpha}}\right)$

$$
\begin{aligned}
& \vec{T}^{a}=X^{b} \epsilon_{b}{ }^{a} \partial_{\phi}+\left(v+\frac{1}{2} \chi^{2} v_{2}\right) \epsilon^{a b} \partial_{b}-\left(\chi F^{a}\right)^{\beta} \partial_{\beta} \\
& \vec{T}^{\alpha}=-\frac{1}{2}\left(\chi \gamma^{3}\right)^{\alpha} \partial_{\phi}+\left(\chi F^{b}\right)^{\alpha} \partial_{b}+\left(v^{\alpha \beta}+\frac{1}{2} \chi^{2} v_{2}^{\alpha \beta}\right) \partial_{\beta} .
\end{aligned}
$$

To find the solution of (51)-(53) it is necessary to expand in terms of the anticommutative coordinate $\chi^{\alpha}$. Therefore, it is convenient to split off any dependence on $\chi^{\alpha}$ and its derivative also in (54) and (55), using instead the special Lorentz vector and spinor matrix valued derivatives ${ }^{6}$

$$
\begin{aligned}
\nabla^{c} & :=X^{d} \epsilon_{d}^{c} \partial_{\phi}+v \epsilon^{c d} \partial_{d}, \\
\nabla_{\delta}^{\alpha} & :=-\frac{1}{2}\left(\gamma^{3}\right)_{\delta}^{\alpha} \partial_{\phi}+F_{\delta}^{d} \partial_{d} .
\end{aligned}
$$

Then the Jacobi identities, arranged in the order $J^{\alpha \beta c}\left|, J^{\alpha \beta \gamma}\right|_{\chi},\left.J^{\alpha b c}\right|_{\chi}$ and $\left.J^{\alpha \beta c}\right|_{\chi^{2}}$, that is the order of increasing complexity best adapted for our further analysis, read

$$
\begin{gathered}
v^{\alpha) \gamma} F_{\gamma}^{c}{ }^{\beta}+\frac{1}{2} \nabla^{c} v^{\alpha \beta}=0, \\
v_{\delta}^{\alpha} v_{2}^{\beta \gamma}-\nabla_{\delta}^{\alpha} v^{\beta \gamma}+\operatorname{cycl}(\alpha \beta \gamma)=0, \\
v_{\delta}^{\alpha} v_{2}-\nabla_{\delta}^{\alpha} v+\nabla^{c} F_{\delta}^{b \alpha} \epsilon_{b c}-\left(F^{c} F^{b}\right)_{\delta}^{\alpha} \epsilon_{b c}=0, \\
\nabla^{c} v_{2}^{\alpha \beta}-F_{\delta}^{c} \delta v_{2}^{\alpha \beta}+v_{2} \epsilon^{c d} \partial_{d} v^{\alpha \beta}+2 \nabla^{\delta(\alpha \mid} F_{\delta}^{c \mid \beta)}+2 v_{2}^{\alpha) \delta} F_{\delta}^{c}(\beta=0 .
\end{gathered}
$$

All known solutions for $d=2$ supergravity models found in the literature have the remarkable property that the Poisson tensor has (almost everywhere, i. e. except for isolated points) constant rank four, implying exactly one conserved

${ }^{6}$ When (56) acts on an invariant function of $\phi$ and $Y, \nabla^{c}$ essentially reduces to the 'scalar' derivative, introduced in (37). 
Casimir function $C$ [107]. Since the purely bosonic Poisson tensor has (almost everywhere) maximal rank two, this implies that the respective fermionic bracket $\mathcal{P}^{\alpha \beta}$ (or, equivalently, its $\chi$-independent part $v^{\alpha \beta}$ ) must be of full rank if only one Casimir function is present in the fermionic extension. In the following subsection we will consider this case, i. e. we will restrict our attention to (regions in the target space with) invertible $\mathcal{P}^{\alpha \beta}$. For describing the rank we introduce the notation $(B \mid F)$. Here $B$ denotes the rank of the bosonic body of the algebra, $F$ the one of the extension. In this language the nondegenerate case has rank (2|2). The remaining degenerate cases with rank $(2 \mid 0)$ and (2|1) will be analyzed in a second step (Sec. 3.2.2 and Sec. 3.2.3).

\subsubsection{Nondegenerate Fermionic Sector}

When the matrix $v^{\alpha \beta}$ in (49) is nondegenerate, i. e. when its determinant

$$
\Delta:=\operatorname{det}\left(v^{\alpha \beta}\right)=\frac{1}{2} v^{\alpha \beta} v_{\beta \alpha}
$$

is nonzero, for a given bosonic bracket this yields all supersymmetric extensions of maximal total rank. We note in parenthesis that due to the two-dimensionality of the spinor space (and the symmetry of $v^{\alpha \beta}$ ) the inverse matrix to $v^{\alpha \beta}$ is nothing else but $v_{\alpha \beta} / \Delta$, which is used in several intermediary steps below.

The starting point of our analysis of the remaining Jacobi identities $J^{A B C}=0$ will always be a certain ansatz, usually for $v^{\alpha \beta}$. Therefore, it will be essential to proceed in a convenient sequence so as to obtain the restrictions on the remaining coefficient functions in the Poisson tensor with the least effort. This is also important because it turns out that several of these equations are redundant. This sequence has been anticipated in (58)-(61). There are already redundancies contained in the second and third step (eqs. (59) and (60)), while the $\chi^{2}$-part of $J^{\alpha \beta c}=0$ (eq. (61)) turns out to be satisfied identically because of the other equations. It should be noted, though, that this peculiar property of the Jacobi identities is not a general feature, resulting e. g. from some hidden symmetry, it holds true only in the case of a nondegenerate $\mathcal{P}^{\alpha \beta}$ (cf. the discussion of the degenerate cases below).

For fixed (nondegenerate) $v^{\alpha \beta}$, all solutions of (58) are parametrized by a Lorentz vector field $f^{a}$ on the coordinate space $\left(\phi, X^{a}\right)$ :

$$
F_{\alpha}^{c \beta}=\left[f^{c} \epsilon^{\gamma \beta}-\nabla^{c} v^{\gamma \beta}\right] \frac{v_{\gamma \alpha}}{2 \Delta}
$$

Eq. (59) can be solved to determine $v_{2}^{\alpha \beta}$ in terms of $v^{\alpha \beta}$ :

$$
v_{2}^{\alpha \beta}=-\frac{1}{4 \Delta} v_{\gamma}^{\delta}\left[\nabla_{\delta}^{\gamma} v^{\alpha \beta}+\operatorname{cycl}(\alpha \beta \gamma)\right]
$$


Multiplying (60) by $v_{\beta}^{\gamma}$ yields

$$
\Delta \delta_{\beta}{ }^{\alpha} v_{2}=v_{\beta}{ }^{\delta}\left[-\nabla_{\delta}^{\alpha} v+\nabla^{c} F_{\delta}^{b} \epsilon_{b c}-\left(F^{c} F^{b}\right)_{\delta}^{\alpha} \epsilon_{b c}\right] .
$$

The trace of (65) determines $v_{2}$, which is thus seen to depend also on the original bosonic potential $v$ of (13).

Neither the vanishing traces of (65) multiplied with $\gamma^{3}$ or with $\gamma_{a}$, nor the identity (61) provide new restrictions in the present case. This has been checked by extensive computer calculations [114], based upon the explicit parametrization (40)-(48), which were necessary because of the extreme algebraic complexity of this problem. It is a remarkable feature of (63), (64) and (65) that the solution of the Jacobi identities for the nondegenerate case can be obtained from algebraic equations only.

As explained at the end of Sec. 2.2 the fermionic extension of the bosonic Casimir function $c$ can be derived from $\left\{\chi^{\alpha}, C\right\}=0$. The general result for the nondegenerate case we note here for later reference

$$
c_{2}=-\frac{1}{2 \Delta} v_{\alpha}{ }^{\beta}\left(-\frac{1}{2}\left(\gamma^{3}\right)_{\beta}{ }^{\alpha} \partial_{\phi}+F_{\beta}^{d}{ }^{\alpha} \partial_{d}\right) c .
$$

The algebra of full rank (2|2) with the above solution for $F_{\alpha}^{c}{ }^{\beta}, v_{2}{ }^{\alpha \beta}$ and $v_{2}$ depends on 6 independent functions $v, v^{\alpha \beta}$ and $f^{a}$ and their derivatives. The original bosonic model determines the 'potential' $v$ in (2) or (13). Thus the arbitrariness of $v^{\alpha \beta}$ and $f^{a}$ indicates that the supersymmetric extensions, obtained by fermionic extension from the PSM, are far from unique. This has been mentioned already in the previous section and we will further illuminate it in the following one.

\subsubsection{Degenerate Fermionic Sector, Rank (2|0)}

For vanishing rank of $\mathcal{P}^{\alpha \beta}$, i. e. $v^{\alpha \beta}=0$, the identities (58) and (59) hold trivially whereas the other Jacobi identities become complicated differential equations relating $F^{a}, v$ and $v_{2}^{\alpha \beta}$. However, these equations can again be reduced to algebraic ones for these functions when the information on additional Casimir functions is employed, which appear in this case. These have to be of fermionic type with the general ansätze

$$
\begin{aligned}
& C^{(+)}=\chi^{+}\left|\frac{X^{--}}{X^{++}}\right|^{\frac{1}{4}} c_{(+)}, \\
& C^{(-)}=\chi^{-}\left|\frac{X^{--}}{X^{++}}\right|^{-\frac{1}{4}} c_{(-)} .
\end{aligned}
$$

The quotients $X^{--} / X^{++}$assure that $c_{( \pm)}$are Lorentz invariant functions of $\phi$ and $Y$. This is possible because the Lorentz boosts in two dimensions do not mix chiral components and the light cone coordinates $X^{ \pm \pm}$. 
Taking a Lorentz covariant ansatz for the Poisson tensor as specified in Sec. 3.1, $C^{(+)}$and $C^{(-)}$must obey $\left\{X^{a}, C^{(+)}\right\}=\left\{X^{a}, C^{(-)}\right\}=0$. Both expressions are linear in $\chi^{\alpha}$, therefore, the coefficients of $\chi^{\alpha}$ have to vanish separately. This leads to $\left(F^{a}\right)_{-}{ }^{+}=0$ and $\left(F^{a}\right)_{+}{ }^{-}=0$ immediately. With the chosen representation of the $\gamma$-matrices (cf. App. A) it is seen that (45) is restricted to $f^{a b}=0$, i. e. the potentials $f_{(s)}, f_{(t)}, f_{(h)}$ and $f_{(a)}$ have to vanish. A further reduction of the system of equations reveals the further conditions $f_{(11)}=0^{7}$ and

$$
v=4 Y f_{(51)}
$$

This leaves the differential equations for $c_{(+)}$and $c_{(-)}$

$$
\begin{gathered}
\left(\nabla+f_{(12)}+f_{(52)}\right) c_{(+)}=0 \\
\left(\nabla+f_{(12)}-f_{(52)}\right) c_{(-)}=0
\end{gathered}
$$

The brackets $\left\{\chi^{+}, C^{(+)}\right\}$and $\left\{\chi^{-}, C^{(-)}\right\}$are proportional to $\chi^{2}$; the resulting equations require $\tilde{u}_{2}=\hat{u}_{2}=0$. The only surviving term $u_{2}$ of $\mathcal{P}^{\alpha \beta}$ is related to $F^{a}$ via $u_{2}=-f_{(12)}$ as can be derived from $\left\{\chi^{-}, C^{(+)}\right\}=0$ as well as from $\left\{\chi^{+}, C^{(-)}\right\}=0$, which are equations of order $\chi^{2}$ too.

Thus the existence of the fermionic Casimir functions (67) and (68) has lead us to a set of algebraic equations among the potentials of the Lorentz covariant ansatz for the Poisson tensor, and the number of independent potentials has been reduced drastically. The final question, whether the Jacobi identities are already fulfilled with the relations found so far finds a positive answer, and the general Poisson tensor with degenerate fermionic sector, depending on four parameter functions $v(\phi, Y), v_{2}(\phi, Y), f_{(12)}(\phi, Y)$ and $f_{(52)}(\phi, Y)$ reads

$$
\begin{aligned}
\mathcal{P}^{a b} & =\left(v+\frac{1}{2} \chi^{2} v_{2}\right) \epsilon^{a b}, \\
\mathcal{P}^{\alpha b} & =\frac{v}{4 Y} X^{b}\left(\chi \gamma^{3}\right)^{\alpha}-f_{(52)} X^{c} \epsilon_{c}{ }^{b}\left(\chi \gamma^{3}\right)^{\alpha}-f_{(12)} X^{c} \epsilon_{c}{ }^{b} \chi^{\alpha}, \\
\mathcal{P}^{\alpha \beta} & =-\frac{1}{2} \chi^{2} f_{(12)} \gamma^{3 \alpha \beta} .
\end{aligned}
$$

This Poisson tensor possesses three Casimir functions: two fermionic ones defined in regions $Y \neq 0$ according to (67) and (68), where $c_{(+)}$and $c_{(-)}$have to fulfil the first order differential equations (70) and (71), respectively, and one bosonic Casimir function $C$ of the form (36), where $c$ is a solution of the bosonic differential equation (37) - note the definition of $\nabla$ therein - and where $c_{2}$ has to obey

$$
\left(\nabla+2 f_{(12)}\right) c_{2}=v_{2} \partial_{Y} c
$$

\footnotetext{
${ }^{7}$ In fact $f_{(11)}$ vanishes in all cases, i. e. also for rank $(2 \mid 2)$ and $(2 \mid 1)$.
} 
Let us finally emphasize that it was decisive within this subsection to use the information on the existence of Casimir functions. This follows from the property of the bivector $\mathcal{P}^{I J}$ to be surface-forming, which in turn is a consequence of the (graded) Jacobi identity satisfied by the bivector. However, the inverse does not hold in general: Not any surface-forming bivector satisfies the Jacobi identities. Therefore, it was necessary to check their validity in a final step.

\subsubsection{Degenerate Fermionic Sector, Rank (2|1)}

When the fermionic sector has maximal rank one, again the existence of a fermionic Casimir function is very convenient. We start with 'positive chirality'8 . We choose the ansatz (cf. App. A)

$$
\mathcal{P}^{\alpha \beta} \mid=v^{\alpha \beta}=i \tilde{u} X^{c}\left(\gamma_{c} P_{+}\right)^{\alpha \beta}=\left(\begin{array}{cc}
\sqrt{2} \tilde{u} X^{++} & 0 \\
0 & 0
\end{array}\right) .
$$

The most general case of rank (2|1) can be reduced to (76) by a (target space) transformation of the spinors. Negative chirality where $P_{+}$is replaced with $P_{-}$ is considered below. Testing the ansätze (67) and (68) reveals that $C^{(-)}$now again is a Casimir function, but $C^{(+)}$is not. Indeed $\left\{\chi^{+}, C^{(+)}\right\} \mid \propto \tilde{u} c_{(+)} \neq 0$ in general, whereas $\left\{\chi^{+}, C^{(-)}\right\} \mid \equiv 0$ shows that the fermionic Casimir function for positive chirality is $C^{(-)}$, where $c_{(-)}=c_{(-)}(\phi, Y)$ has to fulfil a certain differential equation, to be determined below.

The existence of $C^{(-)}$can be used to obtain information about the unknown components of $\mathcal{P}^{A B}$. Indeed an investigation of $\left\{X^{A}, C^{(-)}\right\}=0$ turns out to be much simpler than trying to get that information directly from the Jacobi identities. The bracket $\left\{X^{a}, C^{(-)}\right\}=0$ results in $\left(F^{a}\right)_{+}{ }^{-}=0$ and from $\left\{\chi^{\alpha}, C^{(-)}\right\}=0$ the relation $v_{2}^{--}=0$ can be derived. This is the reason why the ansatz (45) and (48), retaining (46) and (47), attains the simpler form

$$
\begin{aligned}
F^{a} & =f_{(1)}^{a} \mathbb{1}+i f^{a b}\left(\gamma_{b} P_{+}\right)+f_{(5)}^{a} \gamma^{3}, \\
f^{a b} & =f_{(s)} \eta^{a b}+f_{(t)} X^{a} X^{b} .
\end{aligned}
$$

Likewise for the $\chi^{2}$-component of $\mathcal{P}^{\alpha \beta}$ we set

$$
v_{2}^{\alpha \beta}=i \tilde{u}_{2} X^{c}\left(\gamma_{c} P_{+}\right)^{\alpha \beta}+u_{2}\left(\gamma^{3}\right)^{\alpha \beta} .
$$

Not all information provided by the existence of $C^{(-)}$has been introduced at this point. Indeed using the chiral ansatz (76) together with (77)-(79) the calculation of $\left\{X^{a}, C^{(-)}\right\}=0$ in conjunction with the Jacobi identities $J^{\alpha \beta c} \mid=0$ (cf. (58)) requires $f_{(11)}=0$ and

$$
v=4 Y f_{(51)} .
$$

\footnotetext{
${ }^{8}$ 'Positive chirality' refers to the structure of (76). It does not preclude the coupling to the negative chirality component $\chi^{-}$in other terms. A genuine chiral algebra ( similar to $N=(1,0)$ supergravity) is a special case to be discussed below in Sec. 7.6
} 
It should be noted that the results $f_{(11)}=0$ and (80) follow from $\left\{\chi^{\alpha}, C\right\}=0$ too, where $C$ is a bosonic Casimir function. The remaining equation in $\left\{X^{a}, C^{(-)}\right\}=0$ together with $\left\{\chi^{\alpha}, C^{(-)}\right\}=0$ yields $u_{2}=-f_{(12)}$. With the solution obtained so far any calculation of $\left\{X^{A}, C^{(-)}\right\}=0$ leads to one and only one differential equation (71) which must be satisfied in order that (68) is a Casimir function.

We now turn our attention to the Jacobi identities. The inspection of $J^{++c} \mid=$ 0 (58), $\left.J^{+++}\right|_{\chi}=0$ (59) and $\left.J^{+b c}\right|_{\chi}=0$ (60) leads to the conditions

$$
\begin{aligned}
f_{(52)} & =\frac{1}{2}(\nabla \ln |\tilde{u}|)-f_{(12)}-\frac{v}{4 Y}, \\
\tilde{u}_{2} & =f\left(\partial_{Y} \ln |\tilde{u}|\right)+f_{(t)}, \\
v_{2} & =\left(\nabla+2 f_{(12)}+\left(\partial_{Y} v\right)\right) \frac{f}{\tilde{u}},
\end{aligned}
$$

respectively. In order to simplify the notation we introduced

$$
f=f_{(s)}+2 Y f_{(t)} .
$$

All other components of the Jacobi tensor are found to vanish identically.

The construction of graded Poisson tensors with 'negative chirality', i. e. with fermionic sector of the form

$$
\mathcal{P}^{\alpha \beta} \mid=v^{\alpha \beta}=i \tilde{u} X^{c}\left(\gamma_{c} P_{-}\right)^{\alpha \beta}=\left(\begin{array}{cc}
0 & 0 \\
0 & \sqrt{2} \tilde{u} X^{--}
\end{array}\right),
$$

proceeds by the same steps as for positive chirality. Of course, the relevant fermionic Casimir function is now $C^{(+)}$of $(67)$ and $P_{+}$in (77) and (79) has to be replaced by $P_{-}$. The results $f_{(11)}=0,(80),(82)$ and (83) remain the same, only $f_{(52)}$ acquires an overall minus sign,

$$
f_{(52)}=-\frac{1}{2}(\nabla \ln |\tilde{u}|)+f_{(12)}+\frac{v}{4 Y},
$$

to be inserted in the differential equation $(70)$ for $c_{(+)}$.

The results for graded Poisson tensors of both chiralities can be summarized as (cf. (84))

$$
\begin{aligned}
\mathcal{P}^{a b} & =\left(v+\frac{1}{2} \chi^{2}\left[\nabla+2 f_{(12)}+\left(\partial_{Y} v\right)\right] \frac{f}{\tilde{u}}\right) \epsilon^{a b} \\
\mathcal{P}^{\alpha b} & =\left(\chi F^{b}\right)^{\alpha} \\
\mathcal{P}^{\alpha \beta} & =i\left(\tilde{u}+\frac{1}{2} \chi^{2}\left[f\left(\partial_{Y} \ln |\tilde{u}|\right)+f_{(t)}\right]\right) X^{c}\left(\gamma_{c} P_{ \pm}\right)^{\alpha \beta}-\frac{1}{2} \chi^{2} f_{(12)} \gamma^{3 \alpha \beta} .
\end{aligned}
$$

Eq. (88) reads explicitly

$$
\begin{aligned}
F^{b}=\frac{v}{4 Y}\left(X^{b} \pm X^{c} \epsilon_{c}{ }^{b}\right) \gamma^{3}-2 f_{(12)} X^{c} \epsilon_{c}{ }^{b} P_{\mp} \\
\quad+i f_{(s)}\left(\gamma^{b} P_{ \pm}\right)+i f_{(t)} X^{b} X^{c}\left(\gamma_{c} P_{ \pm}\right) \mp \frac{1}{2}(\nabla \ln |\tilde{u}|) X^{c} \epsilon_{c}^{b} \gamma^{3} .
\end{aligned}
$$


Eqs. (87)-(90) represent the generic solution of the graded $N=1$ Poisson algebra of rank $(2 \mid 1)$. It depends beside $v(\gamma, Y)$ on four parameter functions $\tilde{u}, f_{(12)}, f_{(s)}$ and $f_{(t)}$, all depending on $\phi$ and $Y$.

Each chiral type possesses a bosonic Casimir function $C=c+\frac{1}{2} \chi^{2} c_{2}$, where $c(\phi, Y)$ and $c_{2}(\phi, Y)$ are determined by $\nabla c=0$ and

$$
c_{2}=\frac{f \partial_{Y} c}{\tilde{u}} .
$$

The fermionic Casimir function for positive chirality is $C^{(-)}$and for negative chirality $C^{(+)}$(cf. (68) and $(67)$ ), where $c_{(\mp)}(\phi, Y)$ are bosonic scalar functions solving the same differential equation in both cases when eliminating $f_{(52)}$,

$$
\left(\nabla+2 f_{(12)}+\frac{v}{4 Y}-\frac{1}{2}(\nabla \ln |\tilde{u}|)\right) c_{(\mp)}=0
$$

derived from (71) with (81) and from (70) with (86).

\section{Target space diffeomorphisms}

When subjecting the Poisson tensor of the action (27) to a diffeomorphism

$$
X^{I} \rightarrow \bar{X}^{I}=\bar{X}^{I}(X)
$$

on the target space $\mathcal{N}$, another action of gPSM form is generated with the new Poisson tensor

$$
\overline{\mathcal{P}}^{I J}=\left(\bar{X}^{I} \overleftarrow{\partial}_{K}\right) \mathcal{P}^{K L}\left(\vec{\partial}_{L} \bar{X}^{J}\right)
$$

It must be emphasized that in this manner a different model is created with - in the case of $2 \mathrm{~d}$ gravity theories and their fermionic extensions - in general different bosonic 'body' (and global topology). Therefore, such transformations are a powerful tool to create new models from available ones. This is important, because - as shown in Sec. 3 above - the solution of the Jacobi identities as a rule represents a formidable computational problem. This problem could be circumvented by starting from a simple $\overline{\mathcal{P}}^{I J}(\bar{X})$, whose Jacobi identities have been solved rather trivially. As a next step a transformation (93) is applied. The most general Poisson tensor can be generated by calculating the inverse of the Jacobi matrices

$$
\begin{aligned}
J_{I}{ }^{\bar{J}}(X) & =\vec{\partial}_{I} \bar{X}^{J}, & J_{I}^{\bar{K}}\left(J^{-1}\right)_{\bar{K}}{ }^{J}=\delta_{I}{ }^{J}, \\
I^{\bar{I}}{ }_{J}(X) & =\bar{X}^{I} \overleftarrow{\partial}_{J}, & \left(I^{-1}\right)^{I}{ }_{\bar{K}} I^{\bar{K}}{ }_{J}=\delta_{I}{ }^{J} .
\end{aligned}
$$

According to

$$
P^{I J}(X)=\left.\left(I^{-1}\right)_{\bar{K}}^{I} \bar{P}^{K L}\right|_{\bar{X}(X)}\left(J^{-1}\right)_{\bar{L}}^{J}
$$


the components $\mathcal{P}^{I J}$ of the transformed Poisson tensor are expressed in terms of the coordinates $X^{I}$ without the need to invert (93).

The drawback of this argument comes from the fact that in our problem the (bosonic) part of the 'final' algebra is given, and the inverted version of the procedure described here turns out to be very difficult to implement.

Nevertheless, we construct explicitly the diffeomorphisms connecting the dilaton prepotential superalgebra given in Sec. 5.4 with a prototype Poisson tensor in its simplest form, i. e. with a Poisson tensor with constant components. Coordinates where the nonzero components take the values \pm 1 are called CasimirDarboux coordinates. This immediately provides the explicit solution of the corresponding gPSM too; for details cf. Sec. 8.

In addition, we have found target space diffeomorphisms very useful to incorporate e. g. bosonic models related by conformal transformations. An example of that will be given in Sec. 5.5 where an algebra referring to models without bosonic torsion - the just mentioned dilaton prepotential algebra - can be transformed quite simply to one depending quadratically on torsion and thus representing a dilaton theory with kinetic term $(Z \neq 0$ in (3)) in its dilaton version. There the identification $A_{I}=\left(\omega, e_{a}, \psi_{\alpha}\right)$ with 'physical' Cartan variables is used to determine the solution of the latter theory $(Z \neq 0)$ from the simpler model $(\bar{Z}=0)$ with PSM variables $\left(\bar{X}^{I}, \bar{A}_{I}\right)$ by

$$
A_{I}=\left(\vec{\partial}_{I} \bar{X}^{J}\right) \bar{A}_{J}
$$

Also from target space diffeomorphisms interesting information can be collected, regarding the arbitrariness to obtain supersymmetric extensions of bosonic models as found in the general solutions of Sec. 3. Imagine that a certain gPSM has been found, solving the Jacobi identities with a particular ansatz. A natural question would be to find out which other models have the same bosonic body. For this purpose we single out at first all transformations (93) which leave the components $\mathcal{P}^{A \phi}$ form invariant as given by (12) and (16):

$$
\bar{\phi}=\phi, \quad \bar{X}^{a}=X^{b} C_{b}{ }^{a}, \quad \bar{\chi}^{\alpha}=\chi^{\beta} h_{\beta}{ }^{\alpha} .
$$

Here $C_{b}{ }^{a}$ and $h_{\beta}{ }^{\alpha}$ are Lorentz covariant functions (resp. spinor matrices)

$$
\begin{aligned}
& C_{b}{ }^{a}=L \delta_{b}{ }^{a}+M \epsilon_{b}{ }^{a}=c_{b}{ }^{a}+\frac{1}{2} \chi^{2}\left(c_{2}\right)_{b}{ }^{a}, \\
& h_{\beta}{ }^{\alpha}=\left[h_{(1)} \mathbb{1}+h_{(2)} \gamma^{3}+i h_{(3)} X^{c} \gamma_{c}+i h_{(4)} X^{d} \epsilon_{d}{ }^{c} \gamma_{c}\right]_{\beta}{ }^{\alpha},
\end{aligned}
$$

when expressed in terms of $\chi^{2}\left(L=l+\frac{1}{2} \chi^{2} l_{2}\right.$ and similar for $\left.M\right)$ and in terms of $\phi$ and $Y\left(l, l_{2}, m, m_{2}, h_{(i)}\right)$.

The 'stabilisator' $\left(\overline{\mathcal{P}}^{a b}=\mathcal{P}^{a b}\right)$ of the bosonic component $v(\phi, Y)=v(\bar{\phi}, \bar{Y})$ of a graded Poisson tensor will be given by the restriction of $c_{b}{ }^{a}$ to a Lorentz transformation on the target space $\mathcal{N}$ with $l^{2}-m^{2}=1$ in (100). Furthermore 
from the two parameters $h_{(1)}$ and $h_{(2)}$ a Lorentz transformation can be used to reduce them to one independent parameter. Thus no less than five arbitrary two argument functions are found to keep the bosonic part of $\mathcal{P}^{a b}$ unchanged, but produce different fermionic extensions with supersymmetries different from the algebra we started from. This number for rank (2|2) exactly coincides with the number of arbitrary invariant functions found in Sec. 3.2.1. For rank (2|1) in the degenerate case a certain 'chiral' combination of $h_{(3)}$ and $h_{(4)}$ in (101) must be kept fixed, reducing that number to four-again in agreement with Sec. 3.2.3. In a similar way also the appearance of just three arbitrary functions in Sec. 3.2.2 for rank $(2 \mid 0)$ can be understood.

\section{$5 \quad$ Particular Poisson Superalgebras}

The compact formulas of the last sections do not seem suitable for a general discussion, especially in view of the large arbitrariness of gPSMs. We, therefore, elucidate the main features in special models of increasing complexity. The corresponding actions and their relations to the alternative formulations (1) and, or the dilaton theory form (3) will be discussed in Sec. 7.

\subsection{Block Diagonal Algebra}

The most simple ansatz which, nevertheless, already shows the generic features appearing in fermionic extensions, consists in setting the mixed components $\mathcal{P}^{\alpha b}=0$ so that the nontrivial fermionic brackets are restricted to the block $\mathcal{P}^{\alpha \beta}$. Then (58)-(61) reduce to

$$
\begin{gathered}
\nabla^{c} v^{\alpha \beta}=0, \\
v_{\delta}^{\alpha} v_{2}^{\beta \gamma}+\frac{1}{2} \gamma_{\delta}^{3} \partial_{\phi} v^{\beta \gamma}+\operatorname{cycl}(\alpha \beta \gamma)=0, \\
v_{\delta}^{\alpha} v_{2}+\frac{1}{2} \gamma_{\delta}^{3}{ }^{\alpha} \partial_{\phi} v=0, \\
v_{2} \epsilon^{c d} \partial_{d} v^{\alpha \beta}+\nabla^{c} v_{2}^{\alpha \beta}=0 .
\end{gathered}
$$

Eq. (104) implies the spinorial structure

$$
v^{\alpha \beta}=u\left(\gamma^{3}\right)^{\alpha \beta} .
$$

The trace of (102) with $\gamma^{3}$ leads to the condition (37) for $u$, i. e. $u=u(c(\phi, Y))$ depends on the combination of $\phi$ and $Y$ as determined by the bosonic Casimir function.

For $u \neq 0$ the remaining equations (103), (104) and (105) are fulfilled by

$$
v_{2}^{\alpha \beta}=-\frac{\partial_{\phi} u}{2 u}\left(\gamma^{3}\right)^{\alpha \beta}, \quad v_{2}=-\frac{\partial_{\phi} v}{2 u} .
$$


For the present case according to (66) the Casimir function is

$$
C=c-\frac{1}{2} \chi^{2} \frac{\partial_{\phi} c}{2 u(c)}
$$

It is verified easily that

$$
U=u(C)=u(c)+\frac{1}{2} \chi^{2} u_{2}, \quad u_{2}=-\frac{\partial_{\phi} c}{2 u(c)} \frac{d u}{d c} .
$$

Already in this case we observe that in the fermionic extension $\Delta^{-1}, u^{-1}$ from the inverse of $v^{\alpha \beta}$ may introduce singularities. It should be emphasized that $u=u(c)$ is an arbitrary function of $c(\phi, Y)$. Except for $u=u_{0}=$ const (see below) any generic choice of the arbitrary function $u(c)$ by the factors $u^{-1}$ in (107), thus may introduce restrictions on the allowed range of $\phi$ and $Y$ or new singularities on a certain surface where $u(c(\phi, Y))$ vanishes, not present in the purely bosonic bracket. Indeed, these obstructions in certain fermionic extensions are a generic feature of gPSMs. The singularities are seen to be caused here by $\Delta^{-1}$, the inverse of the determinant (62), except for cases with $\Delta=$ const or when special cancellation mechanisms are invoked. Another source for the same phenomenon will appear below in connection with the appearance of a 'prepotential' for $v$. Still, such 'obstructions' can be argued to be rather harmless. We will come back to these issues in several examples below, especially when discussing an explicit solution in Sec. 8.

This complication can be made to disappear by choosing $u=u_{0}=$ const $\neq 0$. Then the fermionic extension $\left(v^{\prime}=\partial_{\phi} v\right)$

$$
\begin{aligned}
\mathcal{P}^{a b} & =\left(v-\frac{1}{4 u_{0}} \chi^{2} v^{\prime}\right) \epsilon^{a b}, \\
\mathcal{P}^{\alpha b} & =0, \\
\mathcal{P}^{\alpha \beta} & =u_{0}\left(\gamma^{3}\right)^{\alpha \beta}
\end{aligned}
$$

does not lead to restrictions on the purely bosonic part $v(\phi, Y)$ of the Poisson tensor, nor does it introduce additional singularities, beside the ones which may already be present in the potential $v(\phi, Y)$. However, then no genuine supersymmetry survives (see Sec. 7.1 below).

It should be noted that all dilaton models mentioned in the introduction can be accommodated in a nontrivial version $u \neq$ const of this gPSM. We shall call the corresponding supergravity actions their 'diagonal extensions'.

\subsection{Nondegenerate Chiral Algebra}

Two further models follow by setting $u=u_{0}=$ const $\neq 0$ and $\hat{u}= \pm \tilde{u}_{0}=$ const. In this way a generalization with full rank of the chiral $N=(1,0)$ and $N=(0,1)$ 
algebras is obtained (cf. App. A)

$$
v^{\alpha \beta}=i \tilde{u}_{0} X^{c}\left(\gamma_{c} P_{ \pm}\right)^{\alpha \beta}+u_{0}\left(\gamma^{3}\right)^{\alpha \beta} .
$$

This particular choice for the coefficients of $X^{c}$ in $v^{\alpha \beta}$ also has the advantage that $X^{c}$ drops out from $\Delta=-u_{0}^{2} / 4$, thus its inverse exists everywhere. Restricting furthermore $f^{c}=0$ we arrive at

$$
F_{\alpha}^{c \beta}=-\frac{i \tilde{u}_{0} v}{2 u_{0}}\left(\gamma^{c} P_{ \pm}\right)_{\alpha}{ }^{\beta}, \quad v_{2}^{\alpha \beta}=0, \quad v_{2}=-\frac{v^{\prime}}{2 u_{0}} .
$$

This yields another graded Poisson tensor for the arbitrary bosonic potential $v(\phi, Y)$

$$
\begin{aligned}
\mathcal{P}^{a b} & =\left(v-\frac{1}{4 u_{0}} \chi^{2} v^{\prime}\right) \epsilon^{a b}, \\
\mathcal{P}^{\alpha b} & =-\frac{i \tilde{u}_{0} v}{2 u_{0}}\left(\chi \gamma^{b} P_{ \pm}\right)^{\alpha}, \\
\mathcal{P}^{\alpha \beta} & =i \tilde{u}_{0} X^{c}\left(\gamma_{c} P_{ \pm}\right)^{\alpha \beta}+u_{0}\left(\gamma^{3}\right)^{\alpha \beta} .
\end{aligned}
$$

Again there are no obstructions for such models corresponding to any bosonic gravity model, given by a particular choice of $v(\phi, Y)$.

The Casimir function (cf. (66)) reads

$$
C=c-\frac{1}{4 u_{0}} \chi^{2} c^{\prime}
$$

where $c$ must obey (37).

\subsection{Deformed Rigid Supersymmetry}

The structure of rigid supersymmetry is encoded within the Poisson tensor by means of the components $v=0$ and (cf. (50))

$$
v^{\alpha \beta}=i \tilde{u}_{0} X^{c} \gamma_{c}^{\alpha \beta}=\left(\begin{array}{cc}
\sqrt{2} \tilde{u}_{0} X^{++} & 0 \\
0 & \sqrt{2} \tilde{u}_{0} X^{--}
\end{array}\right),
$$

where again $\tilde{u}=\tilde{u}_{0}=$ const $\neq 0$. Here $\Delta=2 Y \tilde{u}_{0}^{2}$ and

$$
\frac{1}{\Delta} v_{\alpha \beta}=\frac{i}{2 Y \tilde{u}_{0}} X^{c} \gamma_{c \alpha \beta}=\left(\begin{array}{cc}
\frac{1}{\sqrt{2} \tilde{u}_{0} X^{++}} & 0 \\
0 & \frac{1}{\sqrt{2} \tilde{u}_{0} X^{--}}
\end{array}\right) .
$$

Generalizing this ansatz to $v \neq 0$, the simplest choice $f^{c}=0$ with an arbitrary function $v(\phi, Y)$ (deformed rigid supersymmetry, DRS) yields

$$
F_{\alpha}^{c \beta}=\frac{v}{4 Y} X^{a}\left(\gamma_{a} \gamma^{c} \gamma^{3}\right)_{\alpha}^{\beta}, \quad v_{2}^{\alpha \beta}=\frac{v}{4 Y} \gamma^{3 \alpha \beta}, \quad v_{2}=0,
$$


and thus for $\mathcal{P}^{I J}$

$$
\begin{aligned}
\mathcal{P}^{a b} & =v \epsilon^{a b} \\
\mathcal{P}^{\alpha b} & =\frac{v}{4 Y} X^{c}\left(\chi \gamma_{c} \gamma^{b} \gamma^{3}\right)^{\alpha} \\
\mathcal{P}^{\alpha \beta} & =i \tilde{u}_{0} X^{c}\left(\gamma_{c}\right)^{\alpha \beta}+\frac{1}{2} \chi^{2} \frac{v}{4 Y}\left(\gamma^{3}\right)^{\alpha \beta},
\end{aligned}
$$

and for the Casimir function $C=c$ with (37).

From (122)-(124) it is clear - in contrast to the algebras 5.1 and 5.2 - that this fermionic extension for a generic $v \neq 0$ introduces a possible further singularity at $Y=0$, which cannot be cured by further assumptions on functions which are still arbitrary.

Of course, in order to describe flat space-time, corresponding to the Poisson tensor of rigid supersymmetry, one has to set $v(\phi, Y)=0$. Then the singularity at $Y=0$ in the extended Poisson tensor disappears.

We remark already here that despite the fact that for $v \neq 0$ the corresponding supersymmetrically extended action functional (in contrast to its purely bosonic part) becomes singular at field values $Y \equiv \frac{1}{2} X^{a} X_{a}=0$, we expect that if solutions of the field equations are singular there as well, such singularities will not be relevant if suitable 'physical' observables are considered. We have in mind the analogy to curvature invariants which are not affected by 'coordinate singularities'. We do, however, not intend to prove this statement in detail within the present paper; in Sec. 8 below we shall only shortly discuss the similar singularities, caused by the prepotential in an explicit solution of the related field-theoretical model.

\subsection{Dilaton Prepotential Algebra}

We now assume that the bosonic potential $v$ is restricted to be a function of the dilaton $\phi$ only, $\dot{v}=\partial_{Y} v=0$. Many models of 2 d supergravity, already known in the literature, are contained within algebras of this type, one of which was described in ref. [104-107]. Let deformed rigid supersymmetry of Sec. 5.3 again be the key component of the Poisson tensor (119). Our attempt in Sec. 5.3 to provide a Poisson tensor for arbitrary $v$ built around that component produced a new singularity at $Y=0$ in the fermionic extension. However, the Poisson tensor underlying the model considered in [104-107] was not singular in $Y$. Indeed there exists a mechanism by which this singularity can be cancelled in the general solution (62)-(65), provided the arbitrary functions are chosen in a specific manner.

For this purpose we add to (119), keeping $\tilde{u}=\tilde{u}_{0}=$ const, the fermionic potential $u(\phi)$,

$$
v^{\alpha \beta}=i \tilde{u}_{0} X^{c}\left(\gamma_{c}\right)^{\alpha \beta}+u\left(\gamma^{3}\right)^{\alpha \beta}=\left(\begin{array}{cc}
\sqrt{2} \tilde{u}_{0} X^{++} & -u \\
-u & \sqrt{2} \tilde{u}_{0} X^{--}
\end{array}\right),
$$


with determinant

$$
\Delta=2 Y \tilde{u}_{0}^{2}-u^{2}
$$

The Hamiltonian vector field $\vec{T}^{c}$ in the solution (63) generates a factor $f_{(t)} \neq 0$ in (48). The independent vector field $f^{c}$ can be used to cancel that factor provided one chooses

$$
f^{c}=\frac{1}{2} u^{\prime} X^{c}
$$

Then the disappearance of $f_{(t)}$ is in agreement with the solution given in ref. [106]. The remaining coefficient functions then follow as

$$
\begin{aligned}
F_{\alpha}^{c \beta} & =\frac{1}{2 \Delta}\left(\tilde{u}_{0}^{2} v+u u^{\prime}\right) X^{a}\left(\gamma_{a} \gamma^{c} \gamma^{3}\right)_{\alpha}^{\beta}+\frac{i \tilde{u}_{0}}{2 \Delta}\left(u v+2 Y u^{\prime}\right)\left(\gamma^{c}\right)_{\alpha}{ }^{\beta} \\
v_{2}^{\alpha \beta} & =\frac{1}{2 \Delta}\left(\tilde{u}_{0}^{2} v+u u^{\prime}\right) \gamma^{3 \alpha \beta} \\
v_{2} & =\frac{u v}{2 \Delta^{2}}\left(\tilde{u}_{0}^{2} v+u u^{\prime}\right)+\frac{u u^{\prime}}{2 \Delta^{2}}\left(u v+2 Y u^{\prime}\right)+\frac{1}{2 \Delta}\left(u v^{\prime}+2 Y u^{\prime} \dot{v}+2 Y u^{\prime \prime}\right) .
\end{aligned}
$$

Up to this point the bosonic potential $v$ and the potential $u$ have been arbitrary functions of $\phi$. Demanding now that

$$
\tilde{u}_{0}^{2} v+u u^{\prime}=0
$$

the singularity at $\Delta=0$ is found to be cancelled not only in the respective first terms of (128)-(130), but also in the rest:

$$
\begin{aligned}
v & =-\frac{\left(u^{2}\right)^{\prime}}{2 \tilde{u}_{0}^{2}}, & F_{\alpha}^{c \beta} & =\frac{i u^{\prime}}{2 \tilde{u}_{0}} \gamma_{\alpha}^{c \beta}, \\
v_{2}^{\alpha \beta} & =0, & v_{2} & =\frac{u^{\prime \prime}}{2 \tilde{u}_{0}^{2}} .
\end{aligned}
$$

Furthermore the fermionic potential $u(\phi)$ is seen to be promoted to a "prepotential' for $v(\phi)$. A closer look at (131) with (126) shows that this relation is equivalent to $\nabla \Delta=0$ which happens to be precisely the defining equation (37) of the Casimir function $c(\phi, Y)$ of the bosonic model. The complete Casimir function follows from (66):

$$
c_{2}=\frac{1}{2 \Delta}\left(u \partial_{\phi}+2 Y u^{\prime} \partial_{Y}\right) c
$$

so that

$$
C=\Delta+\frac{1}{2} \chi^{2} u^{\prime}
$$


Thus the Poisson tensor for $v=v(\phi)$, related to $u(\phi)$ by (131), becomes

$$
\begin{aligned}
\mathcal{P}^{a b} & =\frac{1}{2 \tilde{u}_{0}^{2}}\left(-\left(u^{2}\right)^{\prime}+\frac{1}{2} \chi^{2} u^{\prime \prime}\right) \epsilon^{a b}, \\
\mathcal{P}^{\alpha b} & =\frac{i u^{\prime}}{2 \tilde{u}_{0}}\left(\chi \gamma^{b}\right)^{\alpha} \\
\mathcal{P}^{\alpha \beta} & =i \tilde{u}_{0} X^{c}\left(\gamma_{c}\right)^{\alpha \beta}+u\left(\gamma^{3}\right)^{\alpha \beta}
\end{aligned}
$$

which is indeed free from singularities produced by the supersymmetric extension. However, this does not eliminate all pitfalls: Given a bosonic model described by a particular potential $v(\phi)$ where $\phi$ is assumed to take values in the interval $I \subseteq \mathbb{R}$, we have to solve (131) for the prepotential $u(\phi)$, i. e. the quadratic equation

$$
u^{2}=-2 \tilde{u}_{0}^{2} \int_{\phi_{0}}^{\phi} v(\varphi) d \varphi,
$$

which may possess a solution within the real numbers only for a restricted range $\phi \in J$. The interval $J$ may have a nontrivial intersection with $I$ or even none at all. Clearly no restrictions occur if $v$ contains a potential for the dilaton which happens to lead to a negative definite integral on the r. h. s. of (139) for all values of $\phi$ in $I$ (this happens e.g. if $v$ contains only odd powers of $\phi$ with negative prefactors). On the other hand, the domain of $\phi$ is always restricted if $v$ contains even potentials, as becomes immediately clear when viewing the special solutions given in Table 1 . There the different potentials $v(\phi)$ are labelled according to

\begin{tabular}{|l||l|l|}
\hline Model & $v(\phi)=-\frac{\left(u^{2}\right)^{\prime}}{2 \tilde{u}_{0}^{2}}$ & $u(\phi)$ \\
\hline \hline & 0 & $\tilde{u}_{0} \lambda$ \\
String & $-\Lambda$ & $\pm \tilde{u}_{0} \sqrt{2 \Lambda\left(\phi-\phi_{0}\right)}$ \\
JT & $-\lambda^{2} \phi$ & $\tilde{u}_{0} \lambda \phi$ \\
$R^{2}$ & $-\frac{\alpha}{2} \phi^{2}$ & $\pm \tilde{u}_{0} \sqrt{\frac{\alpha}{3}\left(\phi^{3}-\phi_{0}^{3}\right)}$ \\
Howe & $-2 \lambda^{2} \phi^{3}$ & $\tilde{u}_{0} \lambda \phi^{2}$ \\
\hline SRTG & $-\frac{\lambda^{2}}{\sqrt{\phi}}$ & $2 \tilde{u}_{0} \lambda \sqrt[4]{\phi}$ \\
\hline
\end{tabular}

Table 1: Special Dilaton Prepotential Algebras

the models: The string model with $\Lambda=$ const of $[54,115,116,70-78]$, JT is the Jackiw-Teitelboim model (8), $\overline{\mathrm{SRG}}$ the spherically reduced black hole (6) in the conformal description (cf. Sec. 1); the cubic potential appeared in [98], $R^{2}$ gravity is self-explaining. Note that in the case of $\overline{\mathrm{SRG}} I=J=\mathbb{R}_{+}(\phi>0)$, there is already a (harmless) restriction on allowed values of $\phi$ at the purely bosonic level, cf. (6). 
So, as argued above, one may get rid of the singularities at $Y=0$ of supersymmetric extensions obtained in the previous section. In some cases, however, this leads to a restricted range for allowed values of the dilaton, or, alternatively, to complex valued Poisson tensors. Similarly to our expectation of the harmlessness of the above mentioned 1/Y-singularities on the level of the solutions (cf. also [107]), we expect that also complex-valued Poisson tensors are no serious obstacle (both of these remarks apply to the classical analysis only!). In fact, a similar scenario was seen to be harmless (classically) also in the Poisson Sigma formulation of the $G / G$ model for compact gauge groups like $S U(2)$, cf. [80, 117]. We further illustrate these remarks for the class of supergravity models considered in [104-107] at the end of Sec. 8.

\subsection{Bosonic Potential Linear in $Y$}

In order to retain the $Y$-dependence and thus an algebra with bosonic torsion, we take solution (125)-(130) but instead of (131) we may also choose

$$
v=-\frac{\left(u^{2}\right)^{\prime}}{2 \tilde{u}_{0}^{2}}-\frac{\Delta}{2} f,
$$

where $f$ is an arbitrary function of $\phi$ and $Y$. Thanks to the factor $\Delta$ also in this case the fermionic extension does not introduce new singularities at $\Delta=0 .{ }^{9}$ Even if $f$ is a function of $\phi$ only $(\dot{f}=0)$, this model is quadratic in (bosonic) torsion, because of (126). A straightforward calculation using (140) gives

$$
\begin{aligned}
F_{\alpha}^{c \beta} & =-\frac{\tilde{u}_{0}^{2} f}{4} X^{a}\left(\gamma_{a} \gamma^{c} \gamma^{3}\right)_{\alpha}{ }^{\beta}+i\left(\frac{u^{\prime}}{2 \tilde{u}_{0}}-\frac{\tilde{u}_{0} u f}{4}\right)\left(\gamma^{c}\right)_{\alpha}{ }^{\beta} \\
v_{2}^{\alpha \beta} & =-\frac{\tilde{u}_{0}^{2} f}{4} \gamma^{3 \alpha \beta} \\
v_{2} & =\frac{1}{2}\left(\frac{u^{\prime \prime}}{\tilde{u}_{0}^{2}}-u^{\prime} f-\frac{u f^{\prime}}{2}+\frac{\tilde{u}_{0}^{2} u f^{2}}{4}-\frac{2 Y u^{\prime} \dot{f}}{2}\right) .
\end{aligned}
$$

It is worthwhile to note that the present algebra, where the bosonic potential $v$ is of the type (4), can be reached from the algebra of Sec. 5.4 with $\bar{v}=\bar{v}(\bar{\phi})$ by a conformal transformation, i. e. a target space diffeomorphism in the sense of Sec. 4. We use bars to denote quantities and potentials of the algebra of Sec. 5.4, but not for $\tilde{u}_{0}$ because it remains unchanged, i. e. $\bar{v}=-\frac{\left(\bar{u}^{2}\right)^{\prime}}{2 \tilde{u}_{0}^{2}}$. By

$$
\phi=\bar{\phi}, \quad X^{a}=e^{\varphi(\phi)} \bar{X}^{a}, \quad \chi^{\alpha}=e^{\frac{1}{2} \varphi(\phi)} \bar{\chi}^{\alpha},
$$

\footnotetext{
${ }^{9}$ Clearly also in $(140)$ the replacement $\Delta f \rightarrow G(\Delta, \phi, Y)$ with $G(\Delta, \phi, Y) / \Delta$ regular at $\Delta=0$ has a similar effect. But linearity in $\Delta$ is sufficient for our purposes.
} 
the transformed Poisson tensor, expanded in terms of unbarred coefficient functions (cf. Sec. 3.1) becomes

$$
\begin{aligned}
\tilde{u} & =\tilde{u}_{0}, & \tilde{u}_{2} & =0 \\
u & =e^{\varphi} \bar{u}, & u_{2} & =-\frac{1}{2} \varphi^{\prime}, \\
v & =e^{2 \varphi} \bar{v}-2 Y \varphi^{\prime}, & v_{2} & =e^{\varphi} \frac{\bar{u}^{\prime \prime}}{2 \tilde{u}_{0}^{2}}, \\
f_{(12)} & =\frac{1}{2} \varphi^{\prime}, & f_{(51)} & =-\frac{1}{2} \varphi^{\prime}, \\
f_{(s)} & =e^{\varphi} \frac{\bar{u}^{\prime}}{2 \tilde{u}_{0}} & &
\end{aligned}
$$

and $f_{(11)}=f_{(52)}=f_{(t)}=f_{(h)}=0$. When $u(\phi)$ and $\varphi(\phi)$ are taken as basic independent potentials we arrive at

$$
\begin{aligned}
v & =-\frac{1}{2 \tilde{u}_{0}^{2}} e^{2 \varphi}\left(e^{-2 \varphi} u^{2}\right)^{\prime}-2 Y \varphi^{\prime}, \\
v_{2} & =\frac{1}{2 \tilde{u}_{0}^{2}} e^{\varphi}\left(e^{-\varphi} u\right)^{\prime \prime}, \\
f_{(s)} & =\frac{1}{2 \tilde{u}_{0}} e^{\varphi}\left(e^{-\varphi} u\right)^{\prime} .
\end{aligned}
$$

If we set $\varphi^{\prime}=\tilde{u}_{0}^{2} f / 2$ we again obtain solution (140)-(143) for $Y$-independent $f$. The components $\overline{\mathcal{P}}^{a \phi}$ and $\overline{\mathcal{P}}^{\alpha \phi}$ remain form invariant,

$$
\mathcal{P}^{a \phi}=X^{b} \epsilon_{b}{ }^{a}, \quad \mathcal{P}^{\alpha \phi}=-\frac{1}{2} \chi^{\beta}\left(\gamma^{3}\right)_{\beta}{ }^{\alpha},
$$

in agreement with the requirement determined for this case in Sec. 3.1. For completeness we also list the transformation of the 1 -forms $A_{I}=\left(\omega, e_{a}, \psi_{\alpha}\right)$ according to (98)

$$
\omega=\bar{\omega}-\varphi^{\prime}\left(\bar{X}^{b} \bar{e}_{b}+\frac{1}{2} \bar{\chi}^{\beta} \bar{\psi}_{\beta}\right), \quad e_{a}=e^{-\varphi} \bar{e}_{a}, \quad \psi_{\alpha}=e^{-\frac{1}{2} \varphi} \bar{\psi}_{\alpha}
$$

The second equation in (154) provide the justification for the name 'conformal transformation'.

With the help of the scaling parameter $\varphi$ we can write (150), and also (140), in its equivalent form $\nabla\left(e^{-2 \varphi} \Delta\right)=0$, thus exposing the Casimir function to be $c(\phi, Y)=e^{-2 \varphi} \Delta$. Now $u(\phi)$ and $\varphi(\phi)$ are to be viewed as two independent 
parameter functions labelling specific types of Poisson tensors. The solution

$$
\begin{aligned}
\mathcal{P}^{a b} & =\left(-\frac{1}{2 \tilde{u}_{0}^{2}} e^{2 \varphi}\left(e^{-2 \varphi} u^{2}\right)^{\prime}-2 Y \varphi^{\prime}+\frac{1}{4 \tilde{u}_{0}^{2}} \chi^{2} e^{\varphi}\left(e^{-\varphi} u\right)^{\prime \prime}\right) \epsilon^{a b}, \\
\mathcal{P}^{\alpha b} & =-\frac{1}{2} \varphi^{\prime} X^{a}\left(\chi \gamma_{a} \gamma^{b} \gamma^{3}\right)^{\alpha}+\frac{i}{2 \tilde{u}_{0}} e^{\varphi}\left(e^{-\varphi} u\right)^{\prime}\left(\chi \gamma^{b}\right)^{\alpha}, \\
\mathcal{P}^{\alpha \beta} & =i \tilde{u}_{0} X^{c}\left(\gamma_{c}\right)^{\alpha \beta}+\left(u-\frac{1}{4} \chi^{2} \varphi^{\prime}\right)\left(\gamma^{3}\right)^{\alpha \beta}
\end{aligned}
$$

does not introduce a new singularity at $Y=0$, but in order to provide the extension of the bosonic potential (4) we have to solve (150) for the scaling parameter $\varphi(\phi)$ and the fermionic potential $u(\phi)$, which may again lead to obstructions similar to the ones described at the end of Sec. 5.4. With the integrals over $Z(\phi)$ and $V(\phi)$ introduced in $(39)$ we find

$$
\begin{aligned}
& \varphi=-\frac{1}{2} Q(\phi), \\
& u= \pm \sqrt{-2 \tilde{u}_{0}^{2} e^{-Q(\phi)} W(\phi)} .
\end{aligned}
$$

Now we can read off the restriction to be $W(\phi)<0$, yielding singularities at the boundary $W(\phi)=0$. The ansatz (140) can be rewritten in the equivalent form

$$
\nabla\left(e^{Q} \Delta\right)=0 \Leftrightarrow c(\phi, Y)=e^{Q} \Delta=2 \tilde{u}_{0}^{2}\left(Y e^{Q}+W\right) .
$$

The complete Casimir from (66), which again exhibits the simpler form (134), reads

$$
C=e^{Q}\left(\Delta+\frac{1}{2} \chi^{2} e^{-\frac{1}{2} Q}\left(e^{\frac{1}{2} Q} u\right)^{\prime}\right)
$$

As expected from ordinary $2 \mathrm{~d}$ gravity $C$ is conformally invariant.

Expressing the Poisson tensor in terms of the potentials $V(\phi)$ and $Z(\phi)$ of the original bosonic theory, and with $u(\phi)$ as in (159) we arrive at

$$
\begin{aligned}
\mathcal{P}^{a b} & =\left(V+Y Z-\frac{1}{2} \chi^{2}\left[\frac{V Z+V^{\prime}}{2 u}+\frac{\tilde{u}_{0}^{2} V^{2}}{2 u^{3}}\right]\right) \epsilon^{a b}, \\
\mathcal{P}^{\alpha b} & =\frac{Z}{4} X^{a}\left(\chi \gamma_{a} \gamma^{b} \gamma^{3}\right)^{\alpha}-\frac{i \tilde{u}_{0} V}{2 u}\left(\chi \gamma^{b}\right)^{\alpha} \\
\mathcal{P}^{\alpha \beta} & =i \tilde{u}_{0} X^{c}\left(\gamma_{c}\right)^{\alpha \beta}+\left(u+\frac{Z}{8} \chi^{2}\right)\left(\gamma^{3}\right)^{\alpha \beta} .
\end{aligned}
$$

As will be shown in Sec. 6.3 this provides a supersymmetrization for all the dilaton theories (3), because it covers all theories (2) with $v$ linear in $Y$. Among these two explicit examples, namely SRG and the KV model, will be treated in more detail now. 


\subsubsection{Spherically Reduced Gravity (SRG), Nondiagonal Extension I}

In contrast to the KV-model below, no obstructions are found when (141)-(143) with $v$ given by (140) is used for SRG. For simplicity we take in (5) the case $d=4$ and obtain $Q(\phi)=-\frac{1}{2} \ln (\phi), W(\phi)=-2 \lambda^{2} \sqrt{\phi}$ and

$$
u=2 \tilde{u}_{0} \lambda \sqrt{\phi}, \quad \varphi=\frac{1}{4} \ln (\phi),
$$

where $u_{0}$ is a constant. Here already the bosonic theory is defined for $\phi>0$ only. From (155)-(157) in the Poisson tensor of SRG

$$
\begin{aligned}
P^{a b} & =\left(-\lambda^{2}-\frac{Y}{2 \phi}-\frac{3 \lambda}{32 \tilde{u}_{0} \phi^{3 / 2}} \chi^{2}\right) \epsilon^{a b}, \\
P^{\alpha b} & =-\frac{1}{8 \phi} X^{c}\left(\chi \gamma_{c} \gamma^{b} \gamma^{3}\right)^{\alpha}+\frac{i \lambda}{4 \sqrt{\phi}}\left(\chi \gamma^{b}\right)^{\alpha}, \\
P^{\alpha \beta} & =i \tilde{u}_{0} X^{c}\left(\gamma_{c}\right)^{\alpha \beta}+\left(2 \tilde{u}_{0} \lambda \sqrt{\phi}-\frac{1}{16 \phi} \chi^{2}\right)\left(\gamma^{3}\right)^{\alpha \beta}
\end{aligned}
$$

the singularity of the bosonic part simply carries over to the extension, without introducing any new restriction for $\phi>0$.

The bosonic part of the Casimir function (161) is proportional to the ADM mass for SRG.

\subsubsection{Katanaev-Volovich Model (KV), Nondiagonal Extension I}

The bosonic potential (7) leads to $Q(\phi)=\alpha \phi$, thus $\varphi=-\frac{\alpha}{2} \phi$, and

$$
W(\phi)=\int_{\phi_{0}}^{\phi} e^{\alpha \eta}\left(\frac{\beta}{2} \eta^{2}-\Lambda\right) d \eta=\left.e^{\alpha \eta}\left[\frac{\beta}{2}\left(\frac{2}{\alpha^{3}}-\frac{2 \eta}{\alpha^{2}}+\frac{\eta^{2}}{\alpha}\right)-\frac{\Lambda}{\alpha}\right]\right|_{\phi_{0}} ^{\phi} .
$$

With $u(\phi)$ calculated according to (159) the Poisson tensor is

$$
\begin{aligned}
\mathcal{P}^{a b} & =\left(\frac{\beta}{2} \phi^{2}-\Lambda+\alpha Y+\frac{1}{2} \chi^{2} v_{2}\right) \epsilon^{a b}, \\
\mathcal{P}^{\alpha b} & =\frac{\alpha}{4} X^{a}\left(\chi \gamma_{a} \gamma^{b} \gamma^{3}\right)_{\alpha}{ }^{\beta}-\frac{i \tilde{u}_{0}}{2 u}\left(\frac{\beta}{2} \phi^{2}-\Lambda\right)\left(\chi \gamma^{b}\right)_{\alpha}{ }^{\beta}, \\
\mathcal{P}^{\alpha \beta} & =i \tilde{u}_{0} X^{c}\left(\gamma_{c}\right)^{\alpha \beta}+\left(u+\frac{\alpha}{8} \chi^{2}\right)\left(\gamma^{3}\right)^{\alpha \beta},
\end{aligned}
$$

with

$$
v_{2}=-\frac{\alpha\left(\frac{\beta}{2} \phi^{2}-\Lambda\right)+\beta \phi}{2 u}-\frac{\tilde{u}_{0}^{2}\left(\frac{\beta}{2} \phi^{2}-\Lambda\right)^{2}}{2 u^{3}}
$$


For general parameters $\alpha, \beta, \Lambda$ from (159) restrictions upon the range of $\phi$ will emerge in general, if we do not allow singular and complex Poisson tensors - even no allowed interval for $\phi$ may be found to exist. In fact, as we see from (173), in the present case, the 'problem' of complex-valued Poisson tensors comes together with the 'singularity-problem'.

On the other hand, for $\beta \leq 0$ and $\Lambda \geq 0$, where at least one of these parameter does not vanish, the integrand in (169) becomes negative definite, leading to the restriction $\phi>\phi_{0}$ with singularities at $\phi=\phi_{0}$. If we further assume $\alpha>0$ we can set $\phi_{0}=-\infty$. In contrast to the torsionless $R^{2}$ model (see Table 1 ) the restriction for this particular case disappears and the fermionic potential becomes

$$
u= \pm \tilde{u}_{0} \sqrt{-\frac{\beta}{\alpha^{3}}\left((1-\alpha \phi)^{2}+1\right)+\frac{2 \Lambda}{\alpha}} .
$$

\subsection{General Prepotential Algebra}

This algebra represents the immediate generalization of the torsionless one of Sec. 5.4, when (125) is taken for $v^{\alpha \beta}$, but now with $u$ depending on both $\phi$ and $Y$. Here also $v=v(\phi, Y)$. Again we have $\Delta=2 Y \tilde{u}_{0}^{2}-u^{2}$. By analogy to the step in Sec. 5.4 we again cancel the $f_{(t)}$ term (cf. (48)) by the choice

$$
f^{c}=\frac{1}{2}(\nabla u) X^{c}
$$

This yields

$$
\begin{aligned}
F_{\alpha}^{c \beta} & =\frac{1}{2 \Delta}\left(\tilde{u}_{0}^{2} v+u \nabla u\right) X^{a}\left(\gamma_{a} \gamma^{c} \gamma^{3}\right)_{\alpha}^{\beta}+\frac{i \tilde{u}_{0}}{2 \Delta}(u v+2 Y \nabla u)\left(\gamma^{c}\right)_{\alpha}^{\beta} \\
v_{2}^{\alpha \beta} & =\frac{1}{2 \Delta}\left(\left(\tilde{u}_{0}^{2} v+u \nabla u\right)+\dot{u}(u v+2 Y \nabla u)\right) \gamma^{3 \alpha \beta} \\
v_{2} & =\frac{u v}{2 \Delta^{2}}\left(\tilde{u}_{0}^{2} v+u \nabla u\right)+\frac{u \nabla u}{2 \Delta^{2}}(u v+2 Y \nabla u)+\frac{1}{2 \Delta}\left(u v^{\prime}+2 Y \dot{v} \nabla u+2 Y \nabla^{2} u\right) .
\end{aligned}
$$

The factors $\Delta^{-1}, \Delta^{-2}$ indicate the appearance of action functional singularities, at values of the fields where $\Delta$ vanishes. Again we have to this point kept $u$ independent of $v$. In this case, even when we relate $v$ and $u$ by imposing e.g.

$$
\tilde{u}_{0}^{2} v+u \nabla u=0 \Leftrightarrow \nabla \Delta=0 \Leftrightarrow c(\phi, Y)=\Delta,
$$

in order to cancel the first terms in (176)-(178), a generic singularity obstruction is seen to persist (previous remarks on similar occasions should apply here, too, however). 


\subsection{Algebra with $\boldsymbol{u}(\phi, Y)$ and $\tilde{\boldsymbol{u}}(\phi, Y)$}

For $v^{\alpha \beta}$ we retain (125), but now with both $u$ and $\tilde{u}$ depending on $\phi$ and $Y$. Again the determinant

$$
\Delta=2 Y \tilde{u}^{2}-u^{2}
$$

will introduce singularities. If we want to cancel the $f_{(t)}$ term (cf. (48)) as we did in Sec. 5.4 and Sec. 5.6, we have to set here

$$
f^{c}=\frac{1}{2 \tilde{u}}(\tilde{u} \nabla u-u \nabla \tilde{u}) X^{c} .
$$

This leads to

$$
F^{c}=-\frac{1}{4}(\nabla \ln \Delta) X^{a} \gamma_{a} \gamma^{c} \gamma^{3}+\frac{1}{2}(\nabla \ln \tilde{u}) X^{c} \gamma^{3}+\frac{i \tilde{u} u}{2 \Delta}\left[v+2 Y\left(\nabla \ln \frac{u}{\tilde{u}}\right)\right] \gamma^{c} .
$$

Again we could try to fix $u$ and $\tilde{u}$ suitably so as to cancel e. g. the first term in (182):

$$
-\frac{1}{2} \nabla \Delta=\tilde{u}^{2} v+u \nabla u-2 Y \tilde{u} \nabla \tilde{u}=0 .
$$

But then the singularity obstruction resurfaces in (cf. (183))

$$
v=\frac{\Delta^{\prime}}{\dot{\Delta}}=\frac{-u u^{\prime}+2 Y \tilde{u} \tilde{u}^{\prime}}{\tilde{u}^{2}-u \dot{u}+2 Y \tilde{u} \dot{\tilde{u}}} .
$$

Eq. (182) becomes

$$
F^{c}=\frac{1}{\dot{\Delta}}\left(\tilde{u} \tilde{u}^{\prime}-\frac{u}{\tilde{u}}\left(\tilde{u}^{\prime} \dot{u}-\dot{\tilde{u}} u^{\prime}\right)\right) X^{c} \gamma^{3}+\frac{i}{\dot{\Delta}}\left(\tilde{u} u^{\prime}-2 Y\left(\tilde{u}^{\prime} \dot{u}-\dot{\tilde{u}} u^{\prime}\right)\right) \gamma^{c} .
$$

The general formulas for the Poisson tensor are not very illuminating. Instead, we consider two special cases.

\subsubsection{Spherically Reduced Gravity (SRG), Nondiagonal Extension II}

For SRG also e.g. the alternative

$$
v^{\mathrm{SRG}}(\phi, Y)=\frac{\Delta^{\prime}}{\dot{\Delta}}
$$

exists, where $\tilde{u}$ and $u$ are given by

$$
\tilde{u}=\frac{\tilde{u}_{0}}{\sqrt[4]{\phi}}, \quad u=2 \tilde{u}_{0} \lambda \sqrt[4]{\phi}
$$


and $\tilde{u}_{0}=$ const. The Poisson tensor is

$$
\begin{aligned}
P^{a b} & =\left(-\lambda^{2}-\frac{Y}{2 \phi}-\frac{3 \lambda}{32 \tilde{u}_{0} \phi^{5 / 4}} \chi^{2}\right) \epsilon^{a b}, \\
P^{\alpha b} & =-\frac{1}{8 \phi} X^{b}\left(\chi \gamma^{3}\right)^{\alpha}+\frac{i \lambda}{4 \sqrt{\phi}}\left(\chi \gamma^{b}\right)^{\alpha}, \\
P^{\alpha \beta} & =\frac{i \tilde{u}_{0}}{\sqrt[4]{\phi}} X^{c}\left(\gamma_{c}\right)^{\alpha \beta}+2 \tilde{u}_{0} \lambda \sqrt[4]{\phi}\left(\gamma^{3}\right)^{\alpha \beta} .
\end{aligned}
$$

Regarding the absence of obstructions this solution is as acceptable as (and quite similar to) (166)-(168). Together with the diagonal extension implied by Sec. 5.1 and the nondegenerate chiral extension of Sec. 5.2, these four solutions for the extension of the physically motivated $2 \mathrm{~d}$ gravity theory in themselves represent a counterexample to the eventual hope that the requirement for nonsingular, real extensions might yield a unique answer, especially also for a supersymmetric $N=1$ extension of SRG.

\subsubsection{Katanaev-Volovich Model (KV), Nondiagonal Extension II}

Within the fermionic extension treated now also another alternative version of the KV case may be formulated. As for SRG in Sec. 5.7.1 we may identify the bosonic potential (7) with

$$
v^{\mathrm{KV}}(\phi, Y)=\frac{\Delta^{\prime}}{\dot{\Delta}}
$$

Then $\tilde{u}$ and $u$ must be chosen as

$$
\begin{aligned}
& \tilde{u}=\tilde{u}_{0} e^{\frac{\alpha}{2} \phi}, \\
& u= \pm \sqrt{-2 \tilde{u}_{0}^{2} W(\phi)},
\end{aligned}
$$

where $\tilde{u}_{0}=$ const and $W(\phi)$ has been defined in (169). Instead of (170)-(172) we then obtain

$$
\begin{aligned}
\mathcal{P}^{a b} & =\left(\frac{\beta}{2} \phi^{2}-\Lambda+\alpha Y+\frac{1}{2} \chi^{2} v_{2}\right) \epsilon^{a b} \\
\mathcal{P}^{\alpha b} & =\frac{\alpha}{4} X^{b}\left(\chi \gamma^{3}\right)_{\alpha}{ }^{\beta}-\frac{i \tilde{u}}{2 u}\left(\frac{\beta}{2} \phi^{2}-\Lambda\right)\left(\chi \gamma^{b}\right)_{\alpha}{ }^{\beta} \\
\mathcal{P}^{\alpha \beta} & =i \tilde{u} X^{c}\left(\gamma_{c}\right)^{\alpha \beta}+u\left(\gamma^{3}\right)^{\alpha \beta} .
\end{aligned}
$$

with

$$
v_{2}=-\frac{\alpha\left(\frac{\beta}{2} \phi^{2}-\Lambda\right)+\beta \phi}{2 u}-\frac{\tilde{u}^{2}\left(\frac{\beta}{2} \phi^{2}-\Lambda\right)^{2}}{2 u^{3}},
$$

which, however, is beset with the same obstruction problems as the nondiagonal extension I. 


\section{Supergravity Actions}

The algebras discussed in the last section have been selected in view of their application in specific gravitational actions.

\subsection{First Order Formulation}

With the notation introduced in Sec. 3.1, the identification (10), and after a partial integration, the action (27) takes the explicit form (remember $e_{A}=\left(e_{a}, \psi_{\alpha}\right)$ )

$$
L^{\mathrm{gFOG}}=\int_{\mathcal{M}} \phi d \omega+X^{a} D e_{a}+\chi^{\alpha} D \psi_{\alpha}+\frac{1}{2} \mathcal{P}^{A B} e_{B} e_{A},
$$

where the elements of the Poisson structure by expansion in Lorentz covariant components in the notation of Sec. 3.1 can be expressed explicitly as (cf. (41)$(48))$

$$
\begin{aligned}
\frac{1}{2} \mathcal{P}^{A B} e_{B} e_{A}= & -\frac{1}{2} U\left(\psi \gamma^{3} \psi\right)-\frac{i}{2} \widetilde{U} X^{a}\left(\psi \gamma_{a} \psi\right)-\frac{i}{2} \widehat{U} X^{a} \epsilon_{a}^{b}\left(\psi \gamma_{b} \psi\right) \\
& +\left(\chi F^{a} e_{a} \psi\right)+\frac{1}{2} V \epsilon^{b a} e_{a} e_{b} .
\end{aligned}
$$

Here $F^{a}=\left(F^{a}\right)_{\beta}{ }^{\gamma}$, the quantity of (45), provides the direct coupling of $\psi$ and $\chi$, and $D$ is the Lorentz covariant exterior derivative,

$$
D X^{a}=d X^{a}+X^{b} \omega \epsilon_{b}^{a}, \quad D \chi^{\alpha}=d \chi^{\alpha}-\frac{1}{2} \chi^{\beta} \omega\left(\gamma^{3}\right)_{\beta}^{\alpha} .
$$

Of course, at this point the Jacobi identity had not been used as yet to relate the arbitrary functions; hence the action functional (199) is not invariant under a local supersymmetry. On the other hand, when the Jacobi identities restrict those arbitrary functions, the action (198) possesses the local symmetries (30), where the parameters $\epsilon_{I}=\left(l, \epsilon_{a}, \epsilon_{\alpha}\right)$ correspond to Lorentz symmetry, diffeomorphism invariance and, in addition, to supersymmetry, respectively. We, nevertheless, already at this point may list the explicit supersymmetry transformations with parameter $\epsilon_{I}=\left(0,0, \epsilon_{\alpha}\right)$ for the scalar fields,

$$
\begin{aligned}
\delta \phi & =\frac{1}{2}\left(\chi \gamma^{3} \epsilon\right), \\
\delta X^{a} & =-\left(\chi F^{a} \epsilon\right), \\
\delta \chi^{\alpha} & =U\left(\gamma^{3} \epsilon\right)^{\alpha}+i \widetilde{U} X^{c}\left(\gamma_{c} \epsilon\right)^{\alpha}+i \widehat{U} X^{d} \epsilon_{d}^{c}\left(\gamma_{c} \epsilon\right)^{\alpha},
\end{aligned}
$$


and also for the gauge fields,

$$
\begin{aligned}
\delta \omega= & U^{\prime}\left(\epsilon \gamma^{3} \psi\right)+i \widetilde{U}^{\prime} X^{b}\left(\epsilon \gamma_{b} \psi\right)+i \widehat{U}^{\prime} X^{a} \epsilon_{a}^{b}\left(\epsilon \gamma_{b} \psi\right)+\left(\chi \partial_{\phi} F^{b} \epsilon\right) e_{b} \\
\delta e_{a}= & i \widetilde{U}\left(\epsilon \gamma_{a} \psi\right)+i \widehat{U} \epsilon_{a}^{b}\left(\epsilon \gamma_{b} \psi\right)+\left(\chi \partial_{a} F^{b} \epsilon\right) e_{b} \\
& +X_{a}\left[\dot{U}\left(\epsilon \gamma^{3} \psi\right)+i \dot{\widetilde{U}} X^{b}\left(\epsilon \gamma_{b} \psi\right)+i \dot{\widehat{U}} X^{b} \epsilon_{b}^{c}\left(\epsilon \gamma_{c} \psi\right)\right] \\
\delta \psi_{\alpha}= & -D \epsilon_{\alpha}+\left(F^{b} \epsilon\right)_{\alpha} e_{b} \\
& +\chi_{\alpha}\left[u_{2}\left(\epsilon \gamma^{3} \psi\right)+i \tilde{u}_{2} X^{b}\left(\epsilon \gamma_{b} \psi\right)+i \hat{u}_{2} X^{a} \epsilon_{a}^{b}\left(\epsilon \gamma_{b} \psi\right)\right]
\end{aligned}
$$

with the understanding that they represent symmetries of the action (198) only after the relations between the still arbitrary functions for some specific algebra are implied. The only transformation independent of those functions is (201).

\subsection{Elimination of the Auxiliary Fields $X^{I}$}

We can eliminate the fields $X^{I}$ by a Legendre transformation. To sketch the procedure, we rewrite the action (27) in a suggestive form as Hamiltonian action principle $\left(d^{2} x=d x^{1} \wedge d x^{0}\right)$

$$
L=\int_{\mathcal{M}} d^{2} x\left(X^{I} \dot{\mathcal{A}}_{I}-\mathcal{H}(X, A)\right),
$$

where $X^{I}$ should be viewed as the 'momenta' conjugate to the 'velocities' $\dot{\mathcal{A}}_{I}=$ $\partial_{0} A_{1 I}-\partial_{1} A_{0 I}$ and $A_{m I}$ as the 'coordinates'. Velocities $\dot{\mathcal{A}}_{I}$ and the 'Hamiltonian' $\mathcal{H}(X, A)=\mathcal{P}^{J K} A_{0 K} A_{1 J}$ are densities in the present definition. The second PSM field equation (29), in the form obtained when varying $X^{I}$ in (207), acts as a Legendre transformation of $\mathcal{H}(X, A)$ with respect to the variables $X^{I}$,

$$
\dot{\mathcal{A}}_{I}=\frac{\vec{\partial} \mathcal{H}(X, A)}{\partial X^{I}}
$$

also justifying the interpretation of $\dot{\mathcal{A}}_{I}$ as conjugate to $X^{I}$. When (208) can be solved for all $X^{I}$, we get $X^{I}=X^{I}(\dot{A}, A)$. Otherwise, not all of the $X^{I}$ can be eliminated and additional constraints $\Phi(\dot{\mathcal{A}}, A)=0$ emerge. In the latter situation the constraints may be used to eliminate some of the gauge fields $A_{I}$ in favour of others. When all $X^{I}$ can be eliminated the Legendre transformed density

$$
\mathcal{F}(\dot{\mathcal{A}}, A)=X^{I}(\dot{\mathcal{A}}, A) \dot{\mathcal{A}}_{I}-\mathcal{H}(X(\dot{\mathcal{A}}, A), A)
$$

follows, as well as the second order Lagrangian action principle

$$
L=\int_{\mathcal{M}} d^{2} x \mathcal{F}(\dot{\mathcal{A}}, A),
$$

where the coordinates $A_{m I}$ must be varied independently. 
The formalism already presented applies to any (graded) PSM. If there is an additional volume form $\epsilon$ on the base manifold $\mathcal{M}$ it may be desirable to work with functions instead of densities. This is also possible if the volume is dynamical as in gravity theories, $\epsilon=\epsilon(A)$, because a redefinition of the velocities $\dot{\mathcal{A}}_{I}$ containing coordinates $A_{m I}$ but not momenta $X^{I}$ is always possible, as long as we can interpret the field equations from varying $X^{I}$ as Legendre transformation. In particular we use $\dot{A}_{I}=\star d A_{I}=\epsilon^{m n} \partial_{n} A_{m I}$ as velocities and $H(X, A)=\star\left(-\frac{1}{2} \mathcal{P}^{J K} A_{K} A_{J}\right)=\frac{1}{2} P^{J K} \epsilon^{m n} A_{n K} A_{m J}$ as Hamiltonian function, yielding

$$
L=\int_{\mathcal{M}} \epsilon\left(X^{I} \dot{A}_{I}-H(X, A)\right) .
$$

Variation of $X^{I}$ leads to

$$
\dot{A}_{I}=\frac{\vec{\partial} H(X, A)}{\partial X^{I}} .
$$

Solving this equation for $X^{I}=X^{I}(\dot{A}, A)$ the Legendre transformed function $F(\dot{A}, A)=X^{I}(\dot{A}, A) \dot{A}_{I}-H(X(\dot{A}, A), A)$ constitutes the action

$$
L=\int_{\mathcal{M}} \epsilon F(\dot{A}, A) .
$$

If the Poisson tensor is linear in the coordinates $\mathcal{P}^{J K}=X^{I} f_{I}^{J K}$, where $f_{I}^{J K}$ are structure constants, (212) cannot be used to solve for $X^{I}$, instead the constraint $\dot{A}_{I}-\frac{1}{2} f_{I}^{J K} \epsilon^{m n} A_{n K} A_{m J}=0$ appears, implying that the field strength of ordinary gauge theory is zero. For nonlinear Poisson tensor we have always the freedom to move $X^{I}$-independent terms of the rhs of (212) to the lhs, thus using this particular type of covariant derivatives as velocities conjugate to the momenta $X^{I}$ in the Legendre transformation. This redefinition can already be done in the initial action (211) leading to a redefinition of the Hamiltonian $H(X, A)$.

In order to bring $2 \mathrm{~d}$ gravity theories into the form (211), but with covariant derivatives, it is desirable to split off $\phi$-components of the Poisson tensor and to define the 'velocities' (cf. (326) and (328) in App. A.1) as

$$
\begin{aligned}
\rho & :=\star d \omega=\epsilon^{m n}\left(\partial_{n} \omega_{m}\right), \\
\tau_{a} & :=\star D e_{a}=\epsilon^{m n}\left(\partial_{n} e_{m a}\right)-\omega_{a}, \\
\sigma_{\alpha} & :=\star D \psi_{\alpha}=\epsilon^{m n}\left(\partial_{n} \psi_{m \alpha}+\frac{1}{2} \omega_{n}\left(\gamma^{3} \psi_{m}\right)_{\alpha}\right) .
\end{aligned}
$$

Here $\rho=R / 2$ is proportional to the Ricci scalar; $\tau_{a}$ and $\sigma_{\alpha}$ are, respectively, the torsion vector and the spinor built from the derivative of the Rarita-Schwinger field. As a consequence the Lorentz connection $\omega_{m}$ is absent in the Hamiltonian,

$$
V\left(\phi, X^{A} ; e_{m A}\right)=\frac{1}{2} \mathcal{P}^{B C} \epsilon^{m n} e_{n C} e_{m B}=\frac{1}{e} \mathcal{P}^{B C} e_{0 C} e_{1 B}
$$


of the supergravity action

$$
L=\int_{\mathcal{M}} \epsilon\left(\phi \rho+X^{a} \tau_{a}+\chi^{\alpha} \sigma_{\alpha}-V\left(\phi, X^{A} ; e_{m A}\right)\right) .
$$

\subsection{Superdilaton Theory}

As remarked already in Sec. 1, first order formulations of (bosonic) 2d gravity (and hence PSMs) allow for an at least on the classical level globally equivalent description of general dilaton theories (3). Here we show that this statement remains valid also in the case of additional supersymmetric partners (i. e. for gPSMs). We simply have to eliminate the Lorentz connection $\omega_{a}$ and the auxiliary field $X^{a}$. Of course, also the validity of an algebraic elimination procedure in the most general case should (and can) be checked by verifying that the correct e. o. m.s also follow from the final action (227) or (228). (Alternatively to the procedure applied below one may also proceed as in [39], performing two 'Gaussian integrals' to eliminate $X^{a}$ and $\tau^{a}$ from the action). In fact, in the present section we will allow also for Poisson structures characterized by a bosonic potential $v$ not necessarily linear in $Y \equiv \frac{1}{2} X^{a} X_{a}$ as in (4).

Variation of $X^{a}$ in (198) yields the torsion equation

$$
\tau_{a}=\frac{1}{2}\left(\partial_{a} \mathcal{P}^{A B}\right) \epsilon^{m n} e_{n B} e_{m A}
$$

From (219) using $\tilde{\omega}_{a}:=\star d e_{a}=\epsilon^{m n}\left(\partial_{n} e_{m a}\right)$ and $\tau_{a}=\tilde{\omega}_{a}-\omega_{a}(\operatorname{cf} .(215))^{10}$ we get

$$
\omega_{a}=\tilde{\omega}_{a}-\frac{1}{2}\left(\partial_{a} \mathcal{P}^{A B}\right) \epsilon^{m n} e_{n B} e_{m A}
$$

Using (220) to eliminate $\omega_{a}$ the separate terms of (198) read

$$
\begin{aligned}
\phi d \omega & =\phi d \tilde{\omega}+\epsilon \epsilon^{a n}\left(\partial_{n} \phi\right) \tau_{a}+\text { total div. } \\
X^{a} D e_{a} & =\epsilon X^{a} \tau_{a}, \\
\chi^{\alpha} D \psi_{\alpha} & =\chi^{\alpha} \tilde{D} \psi_{\alpha}+\frac{1}{2} \epsilon \epsilon^{a n}\left(\chi \gamma^{3} \psi_{n}\right) \tau_{a},
\end{aligned}
$$

where $\tau_{a}=\tau_{a}\left(X^{I}, e_{m A}\right)$ is determined by (219). The action, discarding the boundary term in (221), becomes

$$
\begin{aligned}
L=\int_{\mathcal{M}} d^{2} x e[\phi \tilde{\rho} & +\chi^{\alpha} \tilde{\sigma}_{\alpha}-\frac{1}{2} \mathcal{P}^{A B} \epsilon^{m n} e_{n B} e_{m A} \\
& \left.+\left(X^{a}+\epsilon^{a r} \partial_{r} \phi+\frac{1}{2} \epsilon^{a r}\left(\chi \gamma^{3} \psi_{r}\right)\right) \frac{1}{2}\left(\partial_{a} \mathcal{P}^{A B}\right) \epsilon^{m n} e_{n B} e_{m A}\right] .
\end{aligned}
$$

\footnotetext{
${ }^{10}$ For specific supergravities it may be useful to base this separation upon a different SUSYcovariant Lorentz connection $\breve{\omega}_{a}$ (cf. Sec. 7.4 and 7.5).
} 
Here $\tilde{\rho}$ and $\tilde{\sigma}$ are defined in analogy to (214) and (216), but calculated with $\tilde{\omega}_{a}$ instead of $\omega_{a}$.

To eliminate $X^{a}$ we vary once more with respect to $\delta X^{b}$ :

$$
\left[X^{a}+\epsilon^{a n}\left(\partial_{n} \phi\right)+\frac{1}{2} \epsilon^{a n}\left(\chi \gamma^{3} \psi_{n}\right)\right]\left(\partial_{b} \partial_{a} \mathcal{P}^{A B}\right) \epsilon^{m n} e_{n B} e_{m A}=0
$$

For $\left(\partial_{b} \partial_{a} \mathcal{P}^{A B}\right) \epsilon^{m n} e_{n B} e_{m A} \neq 0$ this leads to the (again algebraic) equation

$$
X^{a}=-\epsilon^{a n}\left[\left(\partial_{n} \phi\right)+\frac{1}{2}\left(\chi \gamma^{3} \psi_{n}\right)\right]
$$

for $X^{a}$. It determines $X^{a}$ in a way which does not depend on the specific gPSM, because (226) is nothing else than the e. o. $\mathrm{m}$. for $\delta \omega$ in (198).

We thus arrive at the superdilaton action for an arbitrary gPSM

$$
L=\int_{\mathcal{M}} \phi d \tilde{\omega}+\chi^{\alpha} \tilde{D} \psi_{\alpha}+\left.\frac{1}{2} \mathcal{P}^{A B}\right|_{X^{a}} e_{B} e_{A},
$$

where $\left.\right|_{X^{a}}$ means that $X^{a}$ has to be replaced by (226). The action (227) expressed in component fields

$$
L=\int_{\mathcal{M}} d^{2} x e\left[\phi \frac{\tilde{R}}{2}+\chi^{\alpha} \tilde{\sigma}_{\alpha}-\left.\frac{1}{2} \mathcal{P}^{A B}\right|_{X^{a}} \epsilon^{m n} e_{n B} e_{m A}\right]
$$

explicitly shows the fermionic generalization of the bosonic dilaton theory (3) for any gPSM. A quadratic term $X^{2}=X^{a} X_{a}$ in $\mathcal{P}^{A B}$ because of the first term in (226) provides the usual kinetic term of the $\phi$-field in (3) if we take the special case (4),

$$
\begin{aligned}
L= & L^{\mathrm{dil}}+L^{\mathrm{f}} \\
L^{\mathrm{f}}= & \int_{\mathcal{M}} d^{2} x e\left[\chi^{\alpha} \tilde{\sigma}_{\alpha}-\frac{Z}{2}\left(\partial^{n} \phi\right)\left(\chi \gamma^{3} \psi_{n}\right)+\frac{Z}{16} \chi^{2}\left(\psi^{n} \psi_{n}\right)\right. \\
& \left.+\left.\frac{1}{2} \chi^{2} v_{2}\right|_{X^{a}}+\left(\left.\chi F^{a}\right|_{X^{a}} \epsilon_{a}^{m} \psi_{m}\right)-\left.\frac{1}{2} \mathcal{P}^{\alpha \beta}\right|_{X^{a}} \epsilon^{m n} \psi_{n \beta} \psi_{m \alpha}\right] .
\end{aligned}
$$

However, (228) even allows an arbitrary dependence on $X^{2}$ and a corresponding dependence on higher powers of $\left(\partial^{n} \phi\right)\left(\partial_{n} \phi\right)$. For the special case where $\mathcal{P}^{A B}$ is linear in $X^{a}$ (224) shows that $X^{a}$ drops out of that action without further elimination. However, the final results (227) and (228) are the same.

\section{Actions for Particular Models}

Whereas in Sec. 5 a broad range of solutions of graded Poisson algebras has been constructed, we now discuss the related actions and their (eventual) relation to 
a supersymmetrization of (1) or (3). It will be found that, in contrast to the transition from (2) to (3) the form (1) of the supersymmetric action requires that the different functions in the gPSM solution obey certain conditions which are not always fulfilled.

For example, in order to obtain the supersymmetrization of (1), $\phi$ and $X^{a}$ should be eliminated by a Legendre transformation. This is possible only if the Hessian determinant of $v(\phi, Y)$ with respect to $X^{i}=\left(\phi, X^{a}\right)$ is regular,

$$
\operatorname{det}\left(\frac{\partial^{2} v}{\partial X^{i} \partial X^{j}}\right) \neq 0 .
$$

Even in that case the generic situation will be that no closed expression of the form (1) can be given.

In the following subsections for each algebra of Sec. 5 the corresponding FOG action (198), the related supersymmetry and examples of the superdilaton version (228) will be presented. In the formulas for the local supersymmetry we always drop the (common) transformation law for $\delta \phi(201)$.

\subsection{Block Diagonal Supergravity}

The action functional can be read off from (198) and (199) for the Poisson tensor of Sec. 5.1 (cf. (109) for $U$ and (107) together with (44) to determine $V$ ). It reads

$$
L^{\mathrm{BDS}}=\int_{\mathcal{M}} \phi d \omega+X^{a} D e_{a}+\chi^{\alpha} D \psi_{\alpha}-\frac{1}{2} U\left(\psi \gamma^{3} \psi\right)+\frac{1}{2} V \epsilon^{b a} e_{a} e_{b}
$$

and according to (201)-(206) possesses the local supersymmetry

$$
\begin{array}{rlrl}
\delta X^{a} & =0, & & \delta \chi^{\alpha}=U\left(\gamma^{3} \epsilon\right)^{\alpha} \\
\delta \omega=U^{\prime}\left(\epsilon \gamma^{3} \psi\right), & \delta e_{a}=X_{a} \dot{U}\left(\epsilon \gamma^{3} \psi\right), & \delta \psi_{\alpha}=-D \epsilon_{\alpha}+\chi_{\alpha} u_{2}\left(\epsilon \gamma^{3} \psi\right) .
\end{array}
$$

This transformation leads to a translation of $\chi^{\alpha}$ on the hypersurface $C=$ const if $u \neq 0$. Comparing with the usual supergravity type symmetry (15) we observe that the first term in $\delta \psi_{\alpha}$ has the required form (15), but the variation $\delta e_{a}$ is quite different.

The fermionic extension (108) of the Casimir function (36) for this class of theories implies an absolute conservation law $C=c_{0}=$ const.

Whether the supersymmetric extension of the action of type (1) can be reached depends on the particular choice of the bosonic potential $v$. An example where the elimination of all target space coordinates $\phi, X^{a}$ and $\chi^{\alpha}$ is feasible and actually quite simple is $R^{2}$-supergravity with $v=-\frac{\alpha}{2} \phi^{2}$ and $U=u_{0}=$ const. ${ }^{11}$ The result

\footnotetext{
${ }^{11}$ Clearly then no genuine supersymmetry is implied by (234). But we use this example as an illustration for a complete elimination of different combinations of $\phi, X^{a}$ and $\chi^{\alpha}$.
} 
in this case is (cf. Sec. 6.2 and especially (218))

$$
L^{R^{2}}=\int_{\mathcal{M}} d^{2} x e\left[\frac{1}{8 \alpha} \tilde{R}^{2}-\frac{2 u_{0}}{\tilde{R}} \tilde{\sigma}^{\alpha} \tilde{\sigma}_{\alpha}+\frac{u_{0}}{2} \epsilon^{n m}\left(\psi_{m} \gamma^{3} \psi_{n}\right)\right] .
$$

Here the tilde in $\tilde{R}$ and $\tilde{\sigma}^{\alpha}$ indicates that the torsion-free connection $\tilde{\omega}_{a}=$ $\epsilon^{m n} \partial_{n} e_{m a}$ is used to calculate the field strengths. In supergravity it is not convenient to eliminate the field $\phi$. Instead it should be viewed as the 'auxiliary field' of supergravity and therefore remain in the action. Thus eliminating only $X^{a}$ and $\chi^{\alpha}$ yields

$$
L^{R^{2}}=\int_{\mathcal{M}} d^{2} x e\left[\phi \frac{\tilde{R}}{2}-\frac{u_{0}}{\alpha \phi} \tilde{\sigma}^{\alpha} \tilde{\sigma}_{\alpha}-\frac{\alpha}{2} \phi^{2}+\frac{u_{0}}{2} \epsilon^{n m}\left(\psi_{m} \gamma^{3} \psi_{n}\right)\right] .
$$

Also for SRG in $d$-dimensions (5) such an elimination is possible if $d \neq 4$, $d<\infty$. However, interestingly enough, the Hessian determinant vanishes just for the physically most relevant dimension four (SRG) and for the DBH (9), preventing just there a transition to the form (1).

The formula for the equivalent superdilaton theories (228) is presented for the restriction (4) to quadratic torsion only, in order to have a direct comparison with (3). The choice $U=u_{0}=$ const yields

$$
L^{\mathrm{BDA}}=L^{\mathrm{dil}}+L^{\mathrm{f}}
$$

with the fermionic extension

$$
\begin{aligned}
L^{\mathrm{f}}= & \int_{\mathcal{M}} d^{2} x e\left[\chi^{\alpha} \tilde{\sigma}_{\alpha}-\frac{Z}{2}\left(\partial^{n} \phi\right)\left(\chi \gamma^{3} \psi_{n}\right)+\frac{Z}{16} \chi^{2}\left(\psi^{n} \psi_{n}\right)\right. \\
& \left.-\frac{1}{4 u_{0}} \chi^{2}\left(V^{\prime}-\frac{Z^{\prime}}{2}\left(\partial^{n} \phi\right)\left(\partial_{n} \phi\right)\right)+\frac{u_{0}}{2} \epsilon^{m n}\left(\psi_{n} \gamma^{3} \psi_{m}\right)\right] .
\end{aligned}
$$

It should be noted that this model represents a superdilaton theory for arbitrary functions $V(\phi)$ and $Z(\phi)$ in $(3)$.

\subsection{Parity Violating Supergravity}

The action corresponding to the algebra of Sec. 5.2 inserted into (198) and (199) becomes

$$
\begin{aligned}
L^{\mathrm{PVS}}=\int_{\mathcal{M}} \phi d \omega+ & X^{a} D e_{a}+\chi^{\alpha} D \psi_{\alpha}+\epsilon\left(v-\frac{1}{4 u_{0}} \chi^{2} v^{\prime}\right) \\
& -\frac{i \tilde{u}_{0} v}{2 u_{0}}\left(\chi \gamma^{a} P_{ \pm} e_{a} \psi\right)-\frac{i \tilde{u}_{0}}{2} X^{a}\left(\psi \gamma_{a} P_{ \pm} \psi\right)-\frac{u_{0}}{2}\left(\psi \gamma^{3} \psi\right),
\end{aligned}
$$


with local supersymmetry

$$
\delta X^{a}=\frac{i \tilde{u}_{0} v}{2 u_{0}}\left(\chi \gamma^{a} P_{ \pm} \epsilon\right), \quad \delta \chi^{\alpha}=i \tilde{u}_{0} X^{b}\left(\gamma_{b} P_{ \pm} \epsilon\right)^{\alpha}+u_{0}\left(\gamma^{3} \epsilon\right)^{\alpha},
$$

as well as

$$
\begin{aligned}
\delta \omega & =-\frac{i \tilde{u}_{0} v^{\prime}}{2 u_{0}}\left(\chi \gamma^{b} P_{ \pm} \epsilon\right) e_{b} \\
\delta e_{a} & =i \tilde{u}_{0}\left(\epsilon \gamma_{a} P_{ \pm} \psi\right)-\frac{i \tilde{u}_{0}}{2 u_{0}} X_{a} \dot{v}\left(\chi \gamma^{b} P_{ \pm} \epsilon\right) e_{b} \\
\delta \psi_{\alpha} & =-D \epsilon_{\alpha}-\frac{i \tilde{u}_{0} v}{2 u_{0}}\left(\gamma^{b} P_{ \pm} \epsilon\right)_{\alpha} e_{b}
\end{aligned}
$$

and the absolute conservation law (118). Here, in contrast to the model of Sec. 7.1, the transformation law of $e_{a}$ essentially has the 'canonical' form (15).

As seen from the action (239) and the symmetry transformations the two chiralities are treated differently, but we do not have the case of a genuine chiral supergravity (cf. Sec. 7.6 below).

\subsection{Deformed Rigid Supersymmetry}

In this case with the algebra (122)-(124) we obtain

$$
\begin{aligned}
L^{\mathrm{DRS}}=\int_{\mathcal{M}} \phi d \omega & +X^{a} D e_{a}+\chi^{\alpha} D \psi_{\alpha}+\epsilon v \\
& +\frac{v}{4 Y} X^{a}\left(\chi \gamma^{3} \gamma_{a} \gamma^{b} e_{b} \psi\right)-\frac{i \tilde{u}_{0}}{2} X^{a}\left(\psi \gamma_{a} \psi\right)-\frac{v}{16 Y} \chi^{2}\left(\psi \gamma^{3} \psi\right)
\end{aligned}
$$

with local supersymmetry (201),

$$
\delta X^{a}=-\frac{v}{4 Y} X^{b}\left(\chi \gamma_{b} \gamma^{a} \gamma^{3} \epsilon\right), \quad \delta \chi^{\alpha}=i \tilde{u}_{0} X^{b}\left(\gamma_{b} \epsilon\right)^{\alpha}+\frac{1}{2} \chi^{2} \frac{v}{4 Y}\left(\gamma^{3} \epsilon\right)^{\alpha},
$$

and

$$
\begin{aligned}
\delta \omega= & \left(\frac{v}{4 Y}\right)^{\prime}\left[X^{c}\left(\chi \gamma_{c} \gamma^{b} \gamma^{3} \epsilon\right) e_{b}+\frac{1}{2} \chi^{2}\left(\epsilon \gamma^{3} \psi\right)\right], \\
\delta e_{a}= & i \tilde{u}_{0}\left(\epsilon \gamma_{a} \psi\right)+\frac{v}{4 Y}\left(\chi \gamma_{a} \gamma^{b} \gamma^{3} \epsilon\right) e_{b} \\
& +X_{a}\left(\frac{v}{4 Y}\right) \cdot\left[X^{c}\left(\chi \gamma_{c} \gamma^{b} \gamma^{3} \epsilon\right) e_{b}+\frac{1}{2} \chi^{2}\left(\epsilon \gamma^{3} \psi\right)\right], \\
\delta \psi_{\alpha}= & -D \epsilon_{\alpha}+\frac{v}{4 Y}\left[X^{c}\left(\gamma_{c} \gamma^{b} \gamma^{3} \epsilon\right)_{\alpha} e_{b}+\chi_{\alpha}\left(\epsilon \gamma^{3} \psi\right)\right] .
\end{aligned}
$$

Clearly, this model exhibits a 'genuine' supergravity symmetry (15). As pointed out already in Sec. 5.3 the bosonic potential $v(\phi, Y)$ is not restricted in any way by the super-extension. However, a new singularity of the action functional occurs at $Y=0$. The corresponding superdilaton theory can be derived along the lines of Sec. 6.3. 


\subsection{Dilaton Prepotential Supergravities}

In its FOG version the action from (198) with (136)-(138) reads

$$
\begin{aligned}
L^{\mathrm{DPA}}=\int_{\mathcal{M}} \phi d \omega+X^{a} D e_{a} & +\chi^{\alpha} D \psi_{\alpha}-\frac{1}{2 \tilde{u}_{0}^{2}} \epsilon\left(\left(u^{2}\right)^{\prime}-\frac{1}{2} \chi^{2} u^{\prime \prime}\right) \\
& +\frac{i u^{\prime}}{2 \tilde{u}_{0}}\left(\chi \gamma^{a} e_{a} \psi\right)-\frac{i \tilde{u}_{0}}{2} X^{a}\left(\psi \gamma_{a} \psi\right)-\frac{u}{2}\left(\psi \gamma^{3} \psi\right),
\end{aligned}
$$

where $\tilde{u}_{0}=$ const and the 'prepotential' $u$ depends on $\phi$ only. The corresponding supersymmetry becomes (201),

$$
\delta X^{a}=-\frac{i u^{\prime}}{2 \tilde{u}_{0}}\left(\chi \gamma^{a} \epsilon\right), \quad \delta \chi^{\alpha}=i \tilde{u}_{0} X^{b}\left(\gamma_{b} \epsilon\right)^{\alpha}+u\left(\gamma^{3} \epsilon\right)^{\alpha}
$$

and further

$$
\begin{aligned}
\delta \omega & =u^{\prime}\left(\epsilon \gamma^{3} \psi\right)+\frac{i u^{\prime \prime}}{2 \tilde{u}_{0}}\left(\chi \gamma^{b} \epsilon\right) e_{b}, \\
\delta e_{a} & =i \tilde{u}_{0}\left(\epsilon \gamma_{a} \psi\right), \\
\delta \psi_{\alpha} & =-D \epsilon_{\alpha}+\frac{i u^{\prime}}{2 \tilde{u}_{0}}\left(\gamma^{b} \epsilon\right)_{\alpha} e_{b} .
\end{aligned}
$$

Here we have the special situation of an action linear in $X^{a}$, described at the end of Sec. 6.3. Variation of $X^{a}$ in (249) leads to the constraint $D e_{a}-\frac{i \tilde{u}_{0}}{2}\left(\psi \gamma_{a} \psi\right)=$ 0 . It can be used to eliminate the Lorentz connection, i. e. $\omega_{a}=\breve{\omega}_{a}$, where we introduced the SUSY-covariant connection

$$
\breve{\omega}_{a}:=\epsilon^{m n}\left(\partial_{n} e_{m a}\right)+\frac{i \tilde{u}_{0}}{2} \epsilon^{m n}\left(\psi_{n} \gamma_{a} \psi_{m}\right) .
$$

The action reads

$$
\begin{aligned}
L^{\mathrm{DPA}}=\int_{\mathcal{M}} d^{2} x e\left[\phi \frac{\breve{R}}{2}+\chi^{\alpha} \breve{\sigma}_{\alpha}-\right. & \frac{1}{2 \tilde{u}_{0}^{2}}\left(\left(u^{2}\right)^{\prime}-\frac{1}{2} \chi^{2} u^{\prime \prime}\right) \\
& \left.+\frac{i u^{\prime}}{2 \tilde{u}_{0}}\left(\chi \gamma^{m} \epsilon_{m}{ }^{n} \psi_{n}\right)+\frac{u}{2} \epsilon^{m n}\left(\psi_{n} \gamma^{3} \psi_{m}\right)\right]
\end{aligned}
$$

where $\breve{R}$ and $\breve{\sigma}$ indicate that these covariant quantities are built with the spinor dependent Lorentz connection (254).

The present model is precisely the one studied in [104-107]. In Sec. 8 we give the explicit solution of the PSM field equations for this model. The $R^{2}$ model and the model of Howe will be treated in a little more detail now. 


\subsection{1 $\quad R^{2}$ Model}

The supersymmetric extension of $R^{2}$-gravity with bosonic potential $v=-\frac{\alpha}{2} \phi^{2}$ is obtained with the general solution $u= \pm \tilde{u}_{0} \sqrt{\frac{\alpha}{3}\left(\phi^{3}-\phi_{0}^{3}\right)}$ (cf. (139)). In order to simplify the analysis we choose $u=\tilde{u}_{0} \sqrt{\frac{\alpha}{3} \phi^{3}}$. The parameter $\alpha$ can have both signs, implying the restriction on the range of the dilaton field such that $\alpha \phi>0$. Thus the superdilaton action (255) becomes

$$
\begin{aligned}
L^{R^{2}}=\int_{\mathcal{M}} d^{2} x e[\phi & \frac{\breve{R}}{2}+\chi^{\alpha} \breve{\sigma}_{\alpha}-\frac{\alpha}{2} \phi^{2}+\frac{1}{16 \tilde{u}_{0}} \chi^{2} \sqrt{\frac{3 \alpha}{\phi}} \\
& \left.+\frac{3 i \alpha}{4} \sqrt{\frac{\phi}{3 \alpha}}\left(\chi \gamma^{m} \epsilon_{m}{ }^{n} \psi_{n}\right)+\frac{\tilde{u}_{0}}{2} \sqrt{\frac{\alpha}{3} \phi^{3}} \epsilon^{m n}\left(\psi_{n} \gamma^{3} \psi_{m}\right)\right] .
\end{aligned}
$$

The equation obtained when varying $\chi^{\alpha}$ yields

$$
\chi^{\alpha}=-8 \tilde{u}_{0} \sqrt{\frac{\phi}{3 \alpha}} \breve{\sigma}^{\alpha}-2 i \tilde{u}_{0}\left(\psi^{n} \epsilon_{n}{ }^{m} \gamma_{m}\right)^{\alpha} .
$$

Eliminating the $\chi^{\alpha}$ field gives

$$
\begin{aligned}
L^{R^{2}}=\int_{\mathcal{M}} d^{2} x e\left[\phi \frac{\breve{R}}{2}-4 \tilde{u}_{0} \sqrt{\frac{\phi}{3 \alpha}}\right. & \breve{\sigma}^{\alpha} \breve{\sigma}_{\alpha}-2 i \tilde{u}_{0} \phi\left(\breve{\sigma} \gamma^{m} \epsilon_{m}{ }^{n} \psi_{n}\right)-\frac{\alpha}{2} \phi^{2} \\
+ & \left.\frac{\tilde{u}_{0}}{4} \sqrt{\frac{\alpha}{3} \phi^{3}}\left(3\left(\psi^{n} \psi_{n}\right)-\epsilon^{m n}\left(\psi_{n} \gamma^{3} \psi_{m}\right)\right)\right] .
\end{aligned}
$$

Further elimination of $\phi$ requires the solution of a cubic equation for $\sqrt{\phi}$ with a complicated explicit solution, leading to an equally complicated supergravity generalization of the formulation (1) of this model.

\subsubsection{Model of Howe}

The supergravity model of Howe [98], originally derived in terms of superfields, is just a special case of our generic model in the graded PSM approach. Using for the various independent potentials the particular values

$$
\tilde{u}_{0}=-2, \quad u=-\phi^{2}
$$

we obtain $\Delta=8 Y-\phi^{4}$ and for the other nonzero potentials (cf. (132), (133))

$$
v=-\frac{1}{2} \phi^{3}, \quad v_{2}=-\frac{1}{4}, \quad f_{(s)}=\frac{1}{2} \phi .
$$


The Lagrange density for this model in the formulation (2) is a special case of (249):

$$
\begin{aligned}
L^{\text {Howe }}= & \int_{\mathcal{M}} \phi d \omega+X^{a} D e_{a}+\chi^{\alpha} D \psi_{\alpha} \\
& +\frac{1}{2} \phi^{2}\left(\psi \gamma^{3} \psi\right)+i X^{a}\left(\psi \gamma_{a} \psi\right)+\frac{i}{2} \phi\left(\chi \gamma^{a} e_{a} \psi\right)-\frac{1}{2} \epsilon\left(\phi^{3}+\frac{1}{4} \chi^{2}\right) .
\end{aligned}
$$

The local supersymmetry transformations from (250)-(253) are

$$
\delta X^{a}=-\frac{i}{2} \phi\left(\chi \gamma^{a} \epsilon\right), \quad \delta \chi^{\alpha}=-\phi^{2}\left(\gamma^{3} \epsilon\right)^{\alpha}-2 i X^{b}\left(\gamma_{b} \epsilon\right)^{\alpha}
$$

and

$$
\begin{aligned}
\delta \omega & =-2 \phi\left(\epsilon \gamma^{3} \psi\right)+\frac{i}{2}\left(\chi \gamma^{b} \epsilon\right) e_{b}, \\
\delta e_{a} & =-2 i\left(\epsilon \gamma_{a} \psi\right), \\
\delta \psi_{\alpha} & =-D \epsilon_{\alpha}+\frac{i}{2} \phi\left(\gamma^{b} \epsilon\right)_{\alpha} e_{b} .
\end{aligned}
$$

Starting from the dilaton action (255) with (259) and (260), the remaining difference to the formulation of Howe is the appearance of the fermionic coordinate $\chi^{\alpha}$. Due to the quadratic term of $\chi^{\alpha}$ in (261) we can use its own algebraic field equations to eliminate it. Applying the Hodge-dual yields

$$
\chi_{\alpha}=4 \breve{\sigma}_{\alpha}+2 i \phi\left(\gamma^{n} \epsilon_{n}{ }^{m} \psi_{m}\right)_{\alpha}
$$

Inserting this into the Lagrange density (261) and into the supersymmetry transformations (201) as well as into (262)-(265) and identifying $\phi$ with the scalar, usually interpreted as auxiliary field $A, \phi \equiv A$, reveals precisely the supergravity model of Howe. That model, in a notation almost identical to the one used here, is also contained in $[101,118]$, where a superfield approach was used.

\subsection{Supergravities with Quadratic Bosonic Torsion}

The algebra (162)-(164) in (198) leads to

$$
\begin{aligned}
L^{\mathrm{QBT}}=\int_{\mathcal{M}} \phi d \omega+ & X^{a} D e_{a}+\chi^{\alpha} D \psi_{\alpha}+\epsilon\left(V+\frac{1}{2} X^{a} X_{a} Z+\frac{1}{2} \chi^{2} v_{2}\right) \\
+ & \frac{Z}{4} X^{a}\left(\chi \gamma^{3} \gamma_{a} \gamma^{b} e_{b} \psi\right)-\frac{i \tilde{u}_{0} V}{2 u}\left(\chi \gamma^{a} e_{a} \psi\right) \\
& -\frac{i \tilde{u}_{0}}{2} X^{a}\left(\psi \gamma_{a} \psi\right)-\frac{1}{2}\left(u+\frac{Z}{8} \chi^{2}\right)\left(\psi \gamma^{3} \psi\right)
\end{aligned}
$$


with $u(\phi)$ determined from $V(\phi)$ and $Z(\phi)$ according to (159) and

$$
v_{2}=-\frac{1}{2 u}\left(V Z+V^{\prime}+\frac{\tilde{u}_{0}^{2} V^{2}}{u^{2}}\right) .
$$

The special interest in models of this type derives from the fact that because of their equivalence to the dilaton theories with dynamical dilaton field (cf. Sec. 6.3) they cover a large class of physically interesting models. Also, as shown in Sec. 5.5 these models are connected by a simple conformal transformation to theories without torsion, discussed in Sec. 7.4.

Regarding the action (267) it should be kept in mind that calculating the prepotential $u(\phi)$ we discovered the condition $W(\phi)<0$ (cf. (159)). This excludes certain bosonic theories from supersymmetrization with real actions, and it may lead to restrictions on $\phi$, but there is even more information contained in this inequality: It leads also to a restriction on $Y$. Indeed, taking into account (38) we find

$$
Y>c(\phi, Y) e^{-Q(\phi)}
$$

The local supersymmetry transformations of the action (267) become (201),

$$
\begin{aligned}
\delta X^{a} & =-\frac{Z}{4} X^{b}\left(\chi \gamma_{b} \gamma^{a} \gamma^{3} \epsilon\right)+\frac{i \tilde{u}_{0} V}{2 u}\left(\chi \gamma^{a} \epsilon\right), \\
\delta \chi^{\alpha} & =i \tilde{u}_{0} X^{b}\left(\gamma_{b} \epsilon\right)^{\alpha}+\left(u+\frac{Z}{8} \chi^{2}\right)\left(\gamma^{3} \epsilon\right)^{\alpha}
\end{aligned}
$$

and

$$
\begin{aligned}
& \delta \omega=\left(-\frac{\tilde{u}_{0}^{2} V}{u}-\frac{Z u}{2}+\frac{Z^{\prime}}{8} \chi^{2}\right)\left(\epsilon \gamma^{3} \psi\right)+\frac{Z^{\prime}}{4} X^{a}\left(\chi \gamma^{3} \gamma_{a} \gamma^{b} \epsilon\right) e_{b} \\
&-\frac{\tilde{u}_{0}}{2 u}\left(V^{\prime}+\frac{V Z}{2}+\frac{\tilde{u}_{0}^{2} V^{2}}{u^{2}}\right)\left(\chi \gamma^{b} \epsilon\right) e_{b}, \\
& \delta e_{a}=i \tilde{u}_{0}\left(\epsilon \gamma_{a} \psi\right)+\frac{Z}{4}\left(\chi \gamma^{3} \gamma_{a} \gamma^{b} \epsilon\right) e_{b}, \\
& \delta \psi_{\alpha}=-D \epsilon_{\alpha}+\frac{Z}{4} X^{a}\left(\gamma^{3} \gamma_{a} \gamma^{b} \epsilon\right)_{\alpha} e_{b}-\frac{i \tilde{u}_{0} V}{2 u}\left(\gamma^{b} \epsilon\right)_{\alpha} e_{b}+\frac{Z}{4} \chi_{\alpha}\left(\epsilon \gamma^{3} \psi\right) .
\end{aligned}
$$

Finally, we take a closer look at the torsion condition. Variation of $X^{a}$ in (267) yields

$$
D e_{a}-\frac{i \tilde{u}_{0}}{2}\left(\psi \gamma_{a} \psi\right)+\frac{Z}{4}\left(\chi \gamma^{3} \gamma_{a} \gamma^{b} e_{b} \psi\right)+\epsilon X_{a} Z=0
$$

For $Z \neq 0$ this can be used to eliminate $X^{a}$ directly, as described in Sec. 6.2 for a generic PSM. The general procedure to eliminate instead $\omega_{a}$ by this equation was outlined in Sec. 6.3. There, covariant derivatives were expressed in terms of 
$\tilde{\omega}_{a}$. For supergravity theories it is desirable to use SUSY-covariant derivatives instead. The standard covariant spinor dependent Lorentz connection $\breve{\omega}_{a}$ was given in (254). However, that quantity does not retain its SUSY-covariance if torsion is dynamical. Eq. (275) provides such a quantity. Taking the Hodge dual, using (215) we find

$$
\begin{aligned}
\omega_{a} & =\breve{\omega}_{a}+X_{a} Z, \\
\breve{\omega}_{a} & \equiv \tilde{\omega}_{a}+\frac{i \tilde{u}_{0}}{2} \epsilon^{m n}\left(\psi_{n} \gamma_{a} \psi_{m}\right)+\frac{Z}{4}\left(\chi \gamma^{3} \gamma_{a} \gamma^{b} \epsilon_{b}{ }^{n} \psi_{n}\right) .
\end{aligned}
$$

Clearly, $\omega_{a}$ possesses the desired properties (cf. (30)), but it is not the minimal covariant connection. The last term in (276) is a function of the target space coordinates $X^{I}$ only, thus covariant by itself, which leads to the conclusion that $\breve{\omega}_{a}$ is the required quantity. Unfortunately, no generic prescription to construct $\breve{\omega}_{a}$ exists however. The rest of the procedure of Sec. 6.3 for the calculation of a superdilaton action starting with (221) still remains valid, but with $\breve{\omega}_{a}$ of $(277)$ replacing $\tilde{\omega}_{a}$.

We point out that it is essential to have the spinor field $\chi^{\alpha}$ in the multiplet; just as $\phi$ has been identified with the usual auxiliary field of supergravity in Sec. 7.4.2, we observe that general supergravity (with torsion) requires an additional auxiliary spinor field $\chi^{\alpha}$.

\subsubsection{Spherically Reduced Gravity (SRG)}

The special case (5) with $d=4$ for the potentials $V$ and $Z$ in (267) yields

$$
\begin{aligned}
L^{\mathrm{SRG}}=\int_{\mathcal{M}} \phi d \omega+ & X^{a} D e_{a}+\chi^{\alpha} D \psi_{\alpha}-\epsilon\left(\lambda^{2}+\frac{1}{4 \phi} X^{a} X_{a}+\frac{3 \lambda}{32 \tilde{u}_{0} \phi^{3 / 2}} \chi^{2}\right) \\
& -\frac{1}{8 \phi} X^{a}\left(\chi \gamma^{3} \gamma_{a} \gamma^{b} e_{b} \psi\right)+\frac{i \lambda}{4 \sqrt{\phi}}\left(\chi \gamma^{a} e_{a} \psi\right) \\
& -\frac{i \tilde{u}_{0}}{2} X^{a}\left(\psi \gamma_{a} \psi\right)-\frac{1}{2}\left(2 \tilde{u}_{0} \lambda \sqrt{\phi}-\frac{1}{16 \phi} \chi^{2}\right)\left(\psi \gamma^{3} \psi\right) .
\end{aligned}
$$

We do not write down the supersymmetry transformations which follow from (270)-(274). We just note that our transformations $\delta e_{a}$ and $\delta \psi_{\alpha}$ are different from the ones obtained in [99]. There, the supergravity multiplet is the same as in the underlying model [98], identical to the one of Sec. 7.4.2. The difference is related to the use of an additional scalar superfield in [99] to construct a superdilaton action. Such an approach lies outside the scope of the present paper, where we remain within pure gPSM without additional fields which, from the point of view of PSM are 'matter' fields.

Here, according to the general derivation of Sec. 6.3, we arrive at the superdilaton action

$$
L^{\mathrm{SRG}}=L^{\mathrm{dil}}+L^{\mathrm{f}},
$$


with bosonic part (3) and the fermionic extension

$$
\begin{aligned}
L^{\mathrm{f}}= & \int_{\mathcal{M}} d^{2} x e\left[\chi^{\alpha} \tilde{\sigma}_{\alpha}+i \tilde{u}_{0}\left\{\left(\partial^{n} \phi\right)+\frac{1}{2}\left(\chi \gamma^{3} \psi^{n}\right)\right\}\left(\psi_{n} \gamma^{m} \psi_{m}\right)\right. \\
& +\frac{1}{8 \phi}\left(\partial^{n} \phi\right)\left(\chi \gamma^{3} \gamma^{m} \gamma_{n} \psi_{m}\right)-\frac{i \lambda}{4 \sqrt{\phi}}\left(\chi \gamma^{3} \gamma^{m} \psi_{m}\right)+\tilde{u}_{0} \lambda \sqrt{\phi} \epsilon^{m n}\left(\psi_{n} \gamma^{3} \psi_{m}\right) \\
& \left.-\frac{1}{32} \chi^{2}\left\{\frac{1}{\phi}\left(\psi^{n} \psi_{n}\right)+\frac{1}{\phi}\left(\psi^{n} \gamma_{n} \gamma^{m} \psi_{m}\right)+\frac{3 \lambda}{\tilde{u}_{0} \phi^{3 / 2}}+\epsilon^{m n}\left(\psi_{n} \gamma^{3} \psi_{m}\right)\right\}\right] .
\end{aligned}
$$

However, as already noted in the previous section, it may be convenient to use the SUSY-covariant $\breve{\omega}_{a}$ (cf. (277)) instead of $\tilde{\omega}_{a}$, with the result:

$$
\begin{aligned}
& L^{\mathrm{SRG}}=\int_{\mathcal{M}} \phi d \breve{\omega}+\chi^{\alpha} \breve{D} \psi_{\alpha}+\frac{\epsilon}{4 \phi}\left[\left(\partial^{n} \phi\right)\left(\partial_{n} \phi\right)+\left(\partial^{n} \phi\right)\left(\chi \gamma^{3} \psi_{n}\right)+\frac{1}{8} \chi^{2}\left(\psi^{n} \psi_{n}\right)\right] \\
& -\epsilon\left[\lambda^{2}+\frac{3 \lambda}{32 \tilde{u}_{0} \phi^{3 / 2}} \chi^{2}\right]+\frac{i \lambda}{4 \sqrt{\phi}}\left(\chi \gamma^{a} e_{a} \psi\right)-\frac{1}{2}\left[2 \tilde{u}_{0} \lambda \sqrt{\phi}-\frac{1}{16 \phi} \chi^{2}\right]\left(\psi \gamma^{3} \psi\right) .
\end{aligned}
$$

\subsubsection{Katanaev-Volovich Model (KV)}

The supergravity action (267) for the algebra of Sec. 5.5.2 reads

$$
\begin{aligned}
L^{\mathrm{KV}}=\int_{\mathcal{M}} d^{2} x e & \phi R+X^{a} \tau_{a}+\chi^{\alpha} \sigma_{\alpha}+\frac{\beta}{2} \phi^{2}-\Lambda+\frac{\alpha}{2} X^{a} X_{a}+\frac{1}{2} \chi^{2} v_{2} \\
+ & \frac{\alpha}{4} X^{a}\left(\chi \gamma_{a} \gamma^{m} \psi_{m}\right)-\frac{i \tilde{u}_{0}\left(\frac{\beta}{2} \phi^{2}-\Lambda\right)}{2 u}\left(\chi \gamma^{m} \epsilon_{m}{ }^{n} \psi_{n}\right) \\
& \left.+\frac{i \tilde{u}_{0}}{2} X^{a} \epsilon^{m n}\left(\psi_{n} \gamma_{a} \psi_{m}\right)+\frac{1}{2}\left(u+\frac{\alpha}{8} \chi^{2}\right) \epsilon^{m n}\left(\psi_{n} \gamma^{3} \psi_{m}\right)\right]
\end{aligned}
$$

where $v_{2}$ and $u$ were given in (173) and (174). It is invariant under the local supersymmetry transformations (201) and

$$
\begin{aligned}
\delta X^{a} & =-\frac{\alpha}{4} X^{b}\left(\chi \gamma_{b} \gamma^{a} \gamma^{3} \epsilon\right)+\frac{i \tilde{u}_{0}\left(\frac{\beta}{2} \phi^{2}-\Lambda\right)}{2 u}\left(\chi \gamma^{a} \epsilon\right) \\
\delta \chi^{\alpha} & =i \tilde{u}_{0} X^{b}\left(\gamma_{b} \epsilon\right)^{\alpha}+\left(u+\frac{\alpha}{8} \chi^{2}\right)\left(\gamma^{3} \epsilon\right)^{\alpha}
\end{aligned}
$$


in conjunction with the transformations

$$
\begin{aligned}
\delta \omega= & {\left[-\frac{\tilde{u}_{0}^{2}\left(\frac{\beta}{2} \phi^{2}-\Lambda\right)}{u}-\frac{\alpha u}{2}\right]\left(\epsilon \gamma^{3} \psi\right) } \\
& -\frac{\tilde{u}_{0}}{2 u}\left[\beta \phi+\frac{\alpha\left(\frac{\beta}{2} \phi^{2}-\Lambda\right)}{2}+\frac{\tilde{u}_{0}^{2}\left(\frac{\beta}{2} \phi^{2}-\Lambda\right)^{2}}{u^{2}}\right]\left(\chi \gamma^{b} \epsilon\right) e_{b}, \\
\delta e_{a}= & i \tilde{u}_{0}\left(\epsilon \gamma_{a} \psi\right)+\frac{\alpha}{4}\left(\chi \gamma^{3} \gamma_{a} \gamma^{b} \epsilon\right) e_{b}, \\
\delta \psi_{\alpha}= & -D \epsilon_{\alpha}+\frac{\alpha}{4} X^{a}\left(\gamma^{3} \gamma_{a} \gamma^{b} \epsilon\right)_{\alpha} e_{b}-\frac{i \tilde{u}_{0}\left(\frac{\beta}{2} \phi^{2}-\Lambda\right)}{2 u}\left(\gamma^{b} \epsilon\right)_{\alpha} e_{b}+\frac{\alpha}{4} \chi_{\alpha}\left(\epsilon \gamma^{3} \psi\right)
\end{aligned}
$$

of the gauge fields.

Also here the explicit formula for the superdilaton action can be obtained easily, however, the complicated formulas are not very illuminating. It turns out that $\delta \omega$ and $\delta \psi_{\alpha}$ contain powers $u^{k}, k=-3, \ldots, 1$. Therefore, singularities related to the prepotential formulae (159) also affect these transformations.

\section{6 $\quad N=(1,0)$ Dilaton Supergravity}

Of special interest among the degenerate algebras of Sec. 3.2.2 and 3.2.3 is the case where one chiral component in $\chi^{\alpha}$ (say $\chi^{-}$) decouples from the theory.

We adopt the solution (87)-(90) for the Poisson algebra accordingly. To avoid a cross term of the form $\left(\chi^{-} \psi_{+}\right)$appearing in $\mathcal{P}^{a \beta} \psi_{\beta} e_{a}=\left(\chi F^{a} e_{a} \psi\right)$ we have to set $f_{(s)}=f_{(t)}=0$. Similarly $f_{(12)}=0$ cancels a $\left(\chi^{-} \chi^{+}\right)$-term in $\frac{1}{2} \mathcal{P}^{\alpha \beta} \psi_{\beta} \psi_{\alpha}$. Furthermore we choose $\tilde{u}=\tilde{u}_{0}=$ const:

$$
\begin{aligned}
L=\int_{\mathcal{M}} \phi d \omega+X^{++} D e_{++} & +X^{--} D e_{--}+\chi^{+} D \psi_{+}+\chi^{-} D \psi_{-} \\
& +\epsilon v+\frac{v}{2 Y} X^{--} e_{--}\left(\chi^{+} \psi_{+}-\chi^{-} \psi_{-}\right)+\frac{\tilde{u}_{0}}{\sqrt{2}} X^{++} \psi_{+} \psi_{+}
\end{aligned}
$$

The chiral components $\chi^{-}$and $\psi_{-}$can be set to zero consistently. The remaining local supersymmetry has one parameter $\epsilon_{+}$only:

$$
\begin{aligned}
\delta \phi & =\frac{1}{2}\left(\chi^{+} \epsilon_{+}\right), & & \\
\delta X^{++} & =0, & \delta X^{--} & =-\frac{v}{2 Y} X^{--}\left(\chi^{+} \epsilon_{+}\right), \\
\delta \chi^{+} & =\sqrt{2} \tilde{u}_{0} X^{++} \epsilon_{+}, & \delta \chi^{-} & =0,
\end{aligned}
$$


and

$$
\begin{aligned}
\delta \omega & =\frac{v^{\prime}}{2 Y} X^{--} e_{--}\left(\chi^{+} \epsilon_{+}\right), \\
\delta e_{++} & =-\sqrt{2} \tilde{u}_{0}\left(\epsilon_{+} \psi_{+}\right)+X_{++}\left(\frac{v}{2 Y}\right) \cdot X^{--} e_{--}\left(\chi^{+} \epsilon_{+}\right), \\
\delta e_{--} & =\frac{v}{2 Y} e_{--}\left(\chi^{+} \epsilon_{+}\right)+X_{--}\left(\frac{v}{2 Y}\right) \cdot X^{--} e_{--}\left(\chi^{+} \epsilon_{+}\right), \\
\delta \psi_{+} & =-D \epsilon_{+}+\frac{v}{2 Y} X^{--} e_{--}, \quad \delta \psi_{-}=0 .
\end{aligned}
$$

\section{Solution of the Dilaton Supergravity Model}

For the dilaton prepotential supergravity model of Sec. 7.4 [104-107] the Poisson algebra was derived in Sec. 5.4. The PSM field equations (28) and (29) simplify considerably in Casimir-Darboux coordinates which can be found explicitly here, as in the pure gravity PSM. This is an improvement as compared to [107] where only the existence of such target coordinates was used.

We start with the Poisson tensor (136)-(138) in the coordinate system $X^{I}=$ $\left(\phi, X^{++}, X^{--}, \chi^{+}, \chi^{-}\right)$. The algebra under consideration has maximal rank $(2 \mid 2)$, implying that there is one bosonic Casimir function $C$. Rescaling (135) we choose here

$$
C=X^{++} X^{--}-\frac{u^{2}}{2 \tilde{u}_{0}^{2}}+\frac{1}{2} \chi^{2} \frac{u^{\prime}}{2 \tilde{u}_{0}^{2}} .
$$

In regions $X^{++} \neq 0$ we use $C$ instead of $X^{--}$as coordinate in target space $\left(X^{--} \rightarrow C\right)$. In regions $X^{--} \neq 0 X^{++} \rightarrow C$ is possible.

Treating the former case explicitly we replace $X^{++} \rightarrow \lambda=-\ln \left|X^{++}\right|$in each of the two patches $X^{++}>0$ and $X^{++}<0$. This function is conjugate to the generator of Lorentz transformation $\phi$ (cf. (12))

$$
\{\lambda, \phi\}=1 \text {. }
$$

The functions $(\phi, \lambda, C)$ constitute a Casimir-Darboux coordinate system for the bosonic sector $[43,79,80]$. Now our aim is to decouple the bosonic sector from the fermionic one. The coordinates $\chi^{\alpha}$ constitute a Lorentz spinor (cf. (16)). With the help of $X^{++}$they can be converted to Lorentz scalars, i. e. $\left\{\tilde{\chi}^{( \pm)}, \phi\right\}=0$,

$$
\chi^{+} \rightarrow \tilde{\chi}^{(+)}=\frac{1}{\sqrt{\left|X^{++}\right|}} \chi^{+}, \quad \chi^{-} \rightarrow \tilde{\chi}^{(-)}=\sqrt{\left|X^{++}\right|} \chi^{-} .
$$

A short calculation for the set of coordinates $\left(\phi, \lambda, C, \tilde{\chi}^{(+)}, \tilde{\chi}^{(-)}\right)$shows that $\left\{\lambda, \tilde{\chi}^{(+)}\right\}=0$ but $\left\{\lambda, \tilde{\chi}^{(-)}\right\}=-\frac{\sigma u^{\prime}}{\sqrt{2} \tilde{u}_{0}} \tilde{\chi}^{(+)}$, where $\sigma:=\operatorname{sign}\left(X^{++}\right)$. The redefinition

$$
\tilde{\chi}^{(-)} \rightarrow \tilde{\tilde{\chi}}^{(-)}=\tilde{\chi}^{(-)}+\frac{\sigma u}{\sqrt{2} \tilde{u}_{0}} \tilde{\chi}^{(+)}
$$


yields $\left\{\lambda, \tilde{\tilde{\chi}}^{(-)}\right\}=0$ and makes the algebra block diagonal. For the fermionic sector

$$
\begin{aligned}
& \left\{\tilde{\chi}^{(+)}, \tilde{\chi}^{(+)}\right\}=\sigma \sqrt{2} \tilde{u}_{0}, \\
& \left\{\tilde{\tilde{\chi}}^{(-)}, \tilde{\tilde{\chi}}^{(-)}\right\}=\sigma \sqrt{2} \tilde{u}_{0} C, \\
& \left\{\tilde{\chi}^{(+)}, \tilde{\tilde{\chi}}^{(-)}\right\}=0
\end{aligned}
$$

is obtained. We first assume that the Casimir function $C$ appearing explicitly on the r.h.s. of (301) is invertible. Then the redefinition

$$
\tilde{\tilde{\chi}}^{(-)} \rightarrow \breve{\chi}^{(-)}=\frac{1}{\sqrt{|C|}} \tilde{\tilde{\chi}}^{(-)}
$$

can be made. That we found the desired Casimir-Darboux system can be seen from

$$
\left\{\breve{\chi}^{(-)}, \breve{\chi}^{(-)}\right\}=s \sigma \sqrt{2} \tilde{u}_{0}
$$

Here $s:=\operatorname{sign}(C)$ denotes the sign of the Casimir function. In fact we could rescale $\breve{\chi}^{(-)}$and $\tilde{\chi}^{(+)}$so as to reduce the respective right hand sides to \pm 1 ; the signature of the fermionic $2 \times 2$ block cannot be changed however. In any case, we call the local coordinates $\bar{X}^{I}:=\left(\phi, \lambda, C, \tilde{\chi}^{(+)}, \breve{\chi}^{(-)}\right)$Casimir-Darboux since the respective Poisson tensor is constant, which is the decisive feature here. Its non-vanishing components can be read off from (297), (300) and (304).

Now it is straightforward to solve the PSM field equations. Bars are used to denote the gauge fields $\bar{A}_{I}=\left(\bar{A}_{\phi}, \bar{A}_{\lambda}, \bar{A}_{C}, \bar{A}_{(+)}, \bar{A}_{(-)}\right)$corresponding to the coordinates $\bar{X}^{I}$. The first PSM e. o.m.s (28) then read

$$
\begin{aligned}
d \phi-\bar{A}_{\lambda} & =0, \\
d \lambda+\bar{A}_{\phi} & =0, \\
d C & =0, \\
d \tilde{\chi}^{(+)}+\sigma \sqrt{2} \tilde{u}_{0} \bar{A}_{(+)} & =0, \\
d \breve{\chi}^{(-)}+s \sigma \sqrt{2} \tilde{u}_{0} \bar{A}_{(-)} & =0 .
\end{aligned}
$$

These equations decompose in two parts (which is true also in the case of several Casimir functions). In regions where the Poisson tensor is of constant rank we obtain the statement that any solution of (28) and (29) lives on symplectic leaves which is expressed here by the differential equation (307) with the one-parameter solution $C=c_{0}=$ const. Eqs. (305)-(309) without (307) are to be used to solve for all gauge fields excluding the ones which correspond to the Casimir functions, thus excluding $\bar{A}_{C}$ in our case. Note that this solution is purely algebraic. The second set of PSM equations (29) again split in two parts. The equations $d \bar{A}_{\phi}=$ 
$d \bar{A}_{\lambda}=d \bar{A}_{(+)}=d \bar{A}_{(-)}=0$ are identically fulfilled, as can be seen from (305)(309). To show this property in the generic case the first PSM equations (28) in conjunction with the Jacobi identity of the Poisson tensor have to be used. The remainder of the second PSM equations are the equations for the gauge fields corresponding to the Casimir functions. In a case as simple as ours we find, together with the local solution in terms of an integration function $F(x)$ (taking values in the commuting supernumbers),

$$
d \bar{A}_{C}=0 \quad \Rightarrow \quad \bar{A}_{C}=-d F .
$$

The explicit solution for the original gauge fields $A_{I}=\left(\omega, e_{a}, \psi_{\alpha}\right)$ is derived from the target space transformation $A_{I}=\left(\partial_{I} \bar{X}^{J}\right) \bar{A}_{J}$, but in order to compare with the case $C=0$ we introduce an intermediate step and give the solution in coordinates $\tilde{X}^{I}=\left(\phi, \lambda, C, \tilde{\chi}^{(+)}, \tilde{\tilde{\chi}}^{(-)}\right)$first. With $\tilde{A}_{I}=\left(\tilde{A}_{\phi}, \tilde{A}_{\lambda}, \tilde{A}_{C}, \tilde{A}_{(+)}, \tilde{A}_{(-)}\right)$ the calculation $\tilde{A}_{I}=\left(\tilde{\partial}_{I} \bar{X}^{J}\right) \bar{A}_{J}$ yields

$$
\tilde{A}_{\phi}=-d \lambda, \quad \tilde{A}_{\lambda}=d \phi, \quad \tilde{A}_{(+)}=-\frac{\sigma}{\sqrt{2} \tilde{u}_{0}} d \tilde{\chi}^{(+)},
$$

and

$$
\tilde{A}_{C}=-d F+\frac{\sigma}{2 \sqrt{2} \tilde{u}_{0} C^{2}} \tilde{\tilde{\chi}}^{(-)} d \tilde{\tilde{\chi}}^{(-)}, \quad \tilde{A}_{(-)}=-\frac{\sigma}{\sqrt{2} \tilde{u}_{0} C} d \tilde{\tilde{\chi}}^{(-)}
$$

This has to be compared with the case $C=0$. Obviously, the fermionic sector is no longer of full rank, and $\tilde{\tilde{\chi}}^{(-)}$is an additional, fermionic Casimir function as seen from (301) and also from the corresponding field equation

$$
d \tilde{\tilde{\chi}}^{(-)}=0 .
$$

Thus, the $\tilde{X}^{I}$ are Casimir-Darboux coordinates on the subspace $C=0$. The PSM e. o. m.s in barred coordinates continue to be of the form (305)-(308). Therefore, the solution (311) remains unchanged, but (312) has to be replaced by the solution of $d \tilde{A}_{C}=0$ and $d \tilde{A}_{(-)}=0$. In terms of the bosonic function $F(x)$ and an additional fermionic function $\rho(x)$ the solution is

$$
\tilde{A}_{C}=-d F, \quad \tilde{A}_{(-)}=-d \rho .
$$

Collecting all formulas the explicit solution for the original gauge fields $A_{I}=$ $\left(\omega, e_{a}, \psi_{\alpha}\right)$ calculated with $A_{I}=\left(\partial_{I} \tilde{X}^{J}\right) \tilde{A}_{J}$ reads (cf. App. A.2 for the definition 
of ++ and -- components of Lorentz vectors)

$$
\begin{aligned}
\omega= & \frac{d X^{++}}{X^{++}}+V \tilde{A}_{C}+\frac{\sigma u^{\prime}}{\sqrt{2} \tilde{u}_{0}} \tilde{\chi}^{(+)} \tilde{A}_{(-)}, \\
e_{++}= & -\frac{d \phi}{X^{++}}+X^{--} \tilde{A}_{C} \\
& +\frac{1}{2 X^{++}}\left[\frac{\sigma}{\sqrt{2} \tilde{u}_{0}} \tilde{\chi}^{(+)} d \tilde{\chi}^{(+)}+\left(\tilde{\chi}^{(-)}-\frac{\sigma u}{\sqrt{2} \tilde{u}_{0}} \tilde{\chi}^{(+)}\right) \tilde{A}_{(-)}\right], \\
e_{--}= & X^{++} \tilde{A}_{C}, \\
\psi_{+}= & -\frac{u^{\prime}}{2 \tilde{u}_{0}^{2}} \chi^{-} \tilde{A}_{C}-\frac{\sigma}{\sqrt{2} \tilde{u}_{0}} \frac{1}{\sqrt{\left|X^{++}\right|}}\left(d \tilde{\chi}^{(+)}-u \tilde{A}_{(-)}\right), \\
\psi_{--}= & \frac{u^{\prime}}{2 \tilde{u}_{0}^{2}} \chi^{+} \tilde{A}_{C}+\sqrt{\left|X^{++}\right|} \tilde{A}_{(-)} .
\end{aligned}
$$

$\tilde{A}_{C}$ and $\tilde{A}_{(-)}$are given by (312) for $C \neq 0$ and by (314) for $C=0$.

For $C \neq 0$ our solution depends on the free function $F$ and the coordinate functions $\left(\phi, X^{++}, X^{--}, \chi^{+}, \chi^{-}\right)$which, however, are constrained by $C=c_{0}=$ const according to (296). For $C=0$ the free functions are $F$ and $\rho$. The coordinate functions $\left(\phi, X^{++}, X^{--}, \chi^{+}, \chi^{-}\right)$here are restricted by $C=0$ in (296) and by $\tilde{\tilde{\chi}}^{(-)}=$const.

This solution holds for $X^{++} \neq 0$; an analogous set of relations can be derived exchanging the role of $X^{++}$and $X^{--}$.

The solution (cf. (316) and (317)) is free from coordinate singularities in the line element, exhibiting a sort of 'super Eddington-Finkelstein' form. For special choices of the potentials $v(\phi)$ or the related prepotential $u(\phi)$ we refer to Table 1 .

This provides also the solution for the models with quadratic bosonic torsion of Sec. 7.5 by a further change of variables (144) with parameter (158). Its explicit form is calculated using (154).

Up to here we did not use the gauge freedom. Actually, in supergravity theories this is generically not that easy, since the fermionic part of the symmetries are known only in their infinitesimal form (the bosonic part corresponds on a global level to diffeomorphisms and local Lorentz transformations). This changes in the present context for the case that Casimir-Darboux coordinates are available. Indeed, for a constant Poisson tensor the otherwise field dependent, nonlinear symmetries (30) can be integrated easily: Within the range of applicability of the target coordinates, $\bar{X}^{I}$ may be shifted by some arbitrary function (except for the Casimir function $C$, which, however, was found to be constant over $\mathcal{M}$ ), and $\bar{A}_{C}$ may be redefined by the addition of some exact part. The only restrictions to these symmetries come from nondegeneracy of the metric (thus e.g. $\tilde{A}_{C}$ should not be put to zero, cf. (317)). In particular we are thus allowed to put both $\tilde{\chi}^{(+)}$and $\breve{\chi}^{(-)}$to zero in the present patch, and thus, if one follows back the transformations introduced, also the original fields $\chi^{ \pm}$. Next, in the patches with 
$X^{++} \neq 0$, we may fix the local Lorentz invariance by $X^{++}:=1$ and the diffeomorphism invariance by choosing $\phi$ and $F$ as local coordinates on the spacetime manifold $\mathcal{M}$. The resulting gauge fixed solution agrees with the one found in the original bosonic theory, cf. e.g. [48]. This is in agreement with the general considerations of [107], here however made explicit.

A final remark concerns the discussion following (139): As noted there, for some choices of the bosonic potential $v$ the potential $u$ becomes complex valued if the range of $\phi$ is not restricted appropriately. It is straightforward to convince oneself that the above formulas are still valid in the case of complex valued potentials $u$ (i. e. complex valued Poisson tensors). Just in intermediary steps, such as due to (299), one used complex valued fields (with some reality constraints). The final gauge fixed solution, however, is not affected by this, being real as it should be.

\section{Summary and Outlook}

The extension of the concept of Poisson Sigma Models (PSM) to the graded case $[104,105,107]$ has been explored in some detail for the application in general two-dimensional supergravity theories, when a dilaton field is present. Adding one $(N=1)$ or more $(N>1)$ pairs of Majorana fields representing respectively a target space (spinor) variable $\chi^{\alpha}$ and a related 'gravitino' $\psi_{m}{ }^{\alpha}$, automatically leads to a supergravity with local supersymmetry closing on-shell. Our approach yields the minimal supermultiplets, avoiding the imposition and evaluation of constraints which is necessary in the superfield formalism. Instead we have to solve Jacobi identities, which the (degenerate) Poisson structure $\mathcal{P}^{A B}$ of a PSM must obey. In our present paper we have performed this task for the full $N=1$ problem. The solution for the algebras turns out to be quite different according to the rank (defined in Sec. 3.2) of the fermionic extension, but could be reduced essentially to an algebraic problem - despite the fact that the Jacobi identities represent a set of nonlinear first order differential equations in terms of the target space coordinates.

In this argument the Casimir functions are found to play a key role. If the fermionic extension is of full rank that function of the corresponding bosonic PSM simply generalizes to a quantity $C$, taking values in the (commuting) supernumbers, because a quadratic contribution of $\chi^{\alpha}$ is included (cf. (36)). If the extension is not of full rank, beside that commuting $C$ also anticommuting Casimir functions of the form (67) and (68) appear.

In certain cases, but not in general, the use of target space diffeomorphisms (cf. Sec. 4) was found to be a useful tool for the construction of the specific algebras and ensuing supergravity models. The study of 'stabilisators', target space transformations which leave an initially given bosonic algebra invariant, also clarified the large arbitrariness (dependence of the solution on arbitrary 
functions) found for the Poisson superalgebras and the respective supergravity actions.

Because of this we have found it advisable to study explicit specialized algebras and supergravity theories of increasing complexity (Sec. 5 and 7). Our examples are chosen in such a way that the extension of known bosonic $2 \mathrm{~d}$ models of gravity, like the Jackiw-Teitelboim model [62-66], the dilaton black-hole [44-51], spherically symmetric gravity, the Katanaev-Volovich model [40,41], $R^{2}$-gravity and others could be covered (cf. Sec. 7). The arbitrariness referred to above has the consequence that in all cases examples of several possible extensions can be given. For a generic supergravity, obtained in this manner, obstructions for the allowed values of the bosonic target space coordinates emerge. Certain extensions are even found to be not viable within real extensions of the bosonic algebra. We identified two sources of these problems: the division by a certain determinant (cf. (62)) in the course of the (algebraic) solution of the Jacobi identities, and the appearance of a 'prepotential' which may be nontrivially related to the potential in the original PSM. The hope for the existence of an eventual criterion for a reduction of the inherent arbitrariness, following from the requirement that such obstructions should be absent, unfortunately did not materialize: e. g. for the physically interesting case of an extension of spherically reduced Einstein gravity no less than four different obstruction-free supergravities are among the examples discussed here, and there exist infinitely more.

The PSM approach for $2 \mathrm{~d}$ gravities contains a preferred formulation of gravity as a 'first order' (in derivatives) action (2) in the bosonic, as well as in the supergravity case (Sec. 6).

In this formulation the target space coordinates $X^{I}=\left(\phi, X^{a}, \chi^{\alpha}\right)$ of the gPSM are seen to coincide with the momenta in a Hamiltonian action. A Hamiltonian analysis is not pursued in our present paper. Instead we discuss the possibility to eliminate $X^{I}$ in part or completely.

The elimination of $X^{a}$ is possible in the action of a generic supergravity PSM together with a torsion dependent part of the spin connection. We show that in this way the most general superdilaton theory with usual bosonic part (3) and minimal content of fermionic fields in its extension (the Majorana spinor $\chi^{\alpha}$ as partner of the dilaton field $\phi$, and the 1-form 'gravitino' $\psi_{\alpha}$ ) is produced.

By contrast, the elimination of the dilaton field $\phi$ and/or the related spinor $\chi^{\alpha}$ can only be achieved in particular cases. Therefore, these fields should be regarded as substantial ingredients when extending a bosonic $2 \mathrm{~d}$ gravity action of the form (1), depending on curvature and torsion.

The supergravity models whose bosonic part is torsion-free $(Z=0$ in (2) with (4), or in (3)) have been studied before [104-107]. Specializing the potential $v(\phi)$ and the extension appropriately, one arrives at the supersymmetric extension of the $R^{2}$-model $\left(v=-\frac{\alpha}{2} \phi^{2}\right)$ and the model of Howe $\left(v=-\frac{1}{2} \phi^{3}\right)$ [98] originally derived in terms of superfields (cf. also [101]). In the latter case the 'auxiliary field' $A$ is found to coincide with the dilaton field $\phi$. It must be emphasized, 
though, that in the PSM approach all these models are obtained by introducing a cancellation mechanism for singularities and ensuing obstructions (for actions and solutions in the real numbers).

When the bosonic model already contains torsion in the PSM form (2) or when, equivalently, $Z \neq 0$ in (3) an extension of a conformal transformation to a target space diffeomorphism in the gPSM allows an appropriate generalization of the models with $Z=0$. Our discussion of spherically symmetric gravity (5) and of the Katanaev-Volovich model (7) show basic differences. Whereas no new obstruction appears for the former 'physical' theory $(\phi>0)$, as already required by (5), the latter model develops a problem with real actions, except when the parameters $\alpha, \beta$ and $\Lambda$ are chosen in a very specific manner.

We also present a field theoretic model for a gPSM with rank (2|1), when only one component of the target space spinor $\chi^{\alpha}$ is involved. Its supersymmetry only contains one anticommuting function so that this class of models can be interpreted as $N=(1,0)$ supergravity.

Finally (Sec. 8) we make the general considerations of [107] more explicit by giving the full (analytic) solution to the class of models summarized in the two preceding paragraphs. It turns out to be sufficient to discuss the case $Z=0$, because $Z \neq 0$ can be obtained by conformal transformation. Our formulation in terms of Casimir-Darboux coordinates (including the fermionic extension) allows the integration of the infinitesimal supersymmetry transformation to finite ones. Within the range of applicability for the target space coordinates $X^{I}$ this permits a gauging of the target space spinors to zero. In this sense supergravities (without matter) are 'trivial'. However, as stressed in the introduction, such arguments break down when (supersymmetric) matter is coupled to the model. This leads us to an outlook on possible further applications.

Clearly starting from any of the models described here, its supertransformations could be used - at least in a trial-and-error manner as in the original $d=4$ supergravity [30-33] — to extend the corresponding bosonic action [106].

The even simpler introduction of matter in the form of a scalar 'testparticle' in gravity explicitly (or implicitly) is a necessary prerequisite for defining the global manifold geometrically to its geodesics (including null directions). We believe that (properly defined) spinning testparticle would be that instrument for $2 \mathrm{~d}$ supergravity. 'Trivial' supergravity should be without influence on its ('super')geodesics. This should work in the same way as coordinate singularities are not felt in bosonic gravity.

Another line of investigation concerns the reduction of $d \geq 4$ supergravities to a $d=2$ effective superdilaton theory. In this way it should be possible to perhaps nail down the large arbitrariness of superdilaton models, when - as in our present work - this problem is regarded from a strictly $d=2$ point of view. It can be verified in different ways [118] that the introduction of Killing spinors within such an approach inevitably leads to complex fermionic (Dirac) components. Thus $2 \mathrm{~d}$ (dilatonic) supergravities with $N \geq 2$ must be considered. As explained in Sec. 2 
also then the gPSM approach seems to be the method of choice. Of course, the increase in the number of fields, together with the restrictions of the additional $S O(N)$ symmetry will provide an even more complicated structure. Already for $N=1$ we had to rely to a large extend on computer-aided techniques.

Preliminary computations show that the 'minimal' supergravity actions, provided by the PSM approach, also seem to be most appropriate for a Hamiltonian analysis leading eventually to a quantum $2 \mathrm{~d}$ supergravity, extending the analogous result for a purely bosonic case [39, 43, 55-57]. The role of the obstruction for real supersymmetric extensions, encountered for some of the models within this paper, has to be reconsidered carefully in this context.

\section{Acknowledgements}

The authors thank H. Balasin, D. Grumiller and M. Volkov for discussions. The project has been supported by project $\mathrm{P} 12.815-\mathrm{TPH}$ and, in its final stage by project P 13.126-PHY of the FWF (Österreichischer Fonds zur Förderung der wissenschaftlichen Forschung). One of the authors (M. E.) is grateful to A. Wipf for hospitality during a research visit at the Friedrich-Schiller-Universität Jena.

\section{A Notation and Identities}

\section{A.1 Forms and Vectors}

Let $x^{m}$ be local coordinates on a manifold $\mathcal{M}$. We define the components of a $p$-form $\Phi$ according to

$$
\Phi=\frac{1}{p !} d x^{m_{p}} \wedge \cdots \wedge d x^{m_{1}} \Phi_{m_{1} \cdots m_{p}}
$$

and the exterior derivative

$$
d \Phi=\frac{1}{p !} d x^{m_{p}} \wedge \cdots \wedge d x^{m_{1}} \wedge d x^{n} \vec{\partial}_{n} \Phi_{m_{1} \cdots m_{p}} .
$$

As a consequence $d$ acts from the right, i. e. for a $q$-form $\Psi$ and a $p$-form $\Phi$ the Leibniz rule is

$$
d(\Psi \wedge \Phi)=\Psi \wedge d \Phi+(-1)^{p} d \Psi \wedge \Phi
$$

This convention is advantageous for the extension to spinors and superspace where we assume similar summations of indices as in (320), (321).

The relation between the right partial derivative $\vec{\partial}_{I}$ and the left partial derivative $\overleftarrow{\partial}_{I}$ for the graded case becomes

$$
\vec{\partial}_{I} f=f \overleftarrow{\partial}_{I}(-1)^{I(f+1)}
$$


where in the exponent $I$ and $f$ are 1 for anticommuting quantities and 0 otherwise.

We use the characters $a, b, c \ldots$ to denote Lorentz indices taking values $(0,1)$. In $d=2$ our Minkowski metric is

$$
\eta_{a b}=\eta^{a b}=\left(\begin{array}{cc}
1 & 0 \\
0 & -1
\end{array}\right)
$$

and for the antisymmetric $\epsilon$-tensor we set $\epsilon_{a b}=\epsilon(a, b)$ and consistently $\epsilon^{a b}=$ $-\epsilon(a, b)$, where $\epsilon(0,1) \equiv 1$ is the $\epsilon$-symbol, so that

$$
\epsilon_{a b}=-\epsilon^{a b}=\left(\begin{array}{cc}
0 & 1 \\
-1 & 0
\end{array}\right)
$$

It obeys $\epsilon_{a}{ }^{b} \epsilon_{b}{ }^{c}=\delta_{a}{ }^{c}$ and $\epsilon_{a b} \epsilon^{c d}=\delta_{a}{ }^{d} \delta_{b}{ }^{c}-\delta_{a}{ }^{c} \delta_{b}{ }^{d}$, and $\epsilon_{a}{ }^{b}$ is the generator of Lorentz transformations in $d=2$. The transition to world indices with the help of the vielbein $e_{a}{ }^{m}$ and its inverse $e_{m}{ }^{a}$ yields

$$
\epsilon_{m n}=e \epsilon(m, n), \quad \epsilon^{m n}=-\frac{1}{e} \epsilon(m, n)
$$

where $e=\operatorname{det}\left(e_{m}{ }^{a}\right)$. We use $\partial_{a}=e_{a}{ }^{m} \partial_{m}$ to denote the moving frame and $e^{a}=d x^{m} e_{m}{ }^{a}$ for the 1 -forms. Thus, the quantity $e_{a}$ used in the PSM context is the 1-form with index lowered, $e_{a}=e^{b} \eta_{b a}$. In terms of the metric $g_{m n}=e_{n}{ }^{b} e_{m}{ }^{a} \eta_{a b}$ and its determinant $g=\operatorname{det}\left(g_{m n}\right)$ we have $e=\sqrt{-g}$. The induced volume form

$$
\epsilon=\frac{1}{2} e^{a} \wedge e^{b} \epsilon_{b a}=e^{1} \wedge e^{0}=e d x^{1} \wedge d x^{0}
$$

enables us to derive the useful relation $d x^{m} \wedge d x^{n}=\epsilon \epsilon^{m n}$, and to define the Hodge dual according to

$$
\star 1=\epsilon, \quad \star d x^{m}=d x^{n} \epsilon_{n}{ }^{m}, \quad \star \epsilon=1 .
$$

It is a linear map, i. e. $\star(\Phi f)=(\star \Phi) f$ for functions $f$ and forms $\Phi$, and bijective $\star \star=\mathrm{id}$.

\section{A.2 Spinors}

The Dirac matrices obey

$$
\gamma^{a} \gamma^{b}+\gamma^{b} \gamma^{a}=2 \eta^{a b}
$$

We use the explicit representation

$$
\gamma_{\alpha}^{0} \beta=\left(\begin{array}{ll}
0 & 1 \\
1 & 0
\end{array}\right), \quad \gamma_{\alpha}^{1}{ }^{\beta}=\left(\begin{array}{cc}
0 & 1 \\
-1 & 0
\end{array}\right),
$$




$$
\gamma^{3}=\gamma^{1} \gamma^{0}=\left(\begin{array}{cc}
1 & 0 \\
0 & -1
\end{array}\right), \quad\left(\gamma^{3}\right)^{2}=\mathbb{1}
$$

Further useful identities are

$$
\begin{gathered}
\gamma^{a} \gamma^{b}=\eta^{a b} \mathbb{1}+\epsilon^{a b} \gamma^{3}, \\
\gamma^{a} \gamma^{3}+\gamma^{3} \gamma^{a}=0, \quad \gamma^{a} \gamma^{3}=\gamma^{b} \epsilon_{b}^{a}, \quad \gamma^{a} \gamma_{b} \gamma_{a}=0,
\end{gathered}
$$

and the Fierz identity

$$
2 \delta_{\alpha}^{\gamma} \delta_{\beta}^{\delta}=\delta_{\alpha}^{\delta} \delta_{\beta}^{\gamma}+\gamma_{\alpha}^{3}{ }^{\delta} \gamma_{\beta}^{3}{ }^{\gamma}+\gamma_{\alpha}^{a}{ }^{\delta} \gamma_{a \beta}^{\gamma}
$$

which implies the completeness relation

$$
\Gamma_{\alpha}^{\beta}=\frac{1}{2} \Gamma_{\gamma}^{\gamma} \delta_{\alpha}^{\beta}+\frac{1}{2}\left(\Gamma \gamma_{a}\right)_{\gamma}^{\gamma} \gamma_{\alpha}^{a}{ }^{\beta}+\frac{1}{2}\left(\Gamma \gamma^{3}\right)_{\gamma}^{\gamma} \gamma_{\alpha}^{3}{ }^{\beta}
$$

For a spinor

$$
\chi_{\alpha}=\left(\begin{array}{l}
\chi_{+} \\
\chi_{-}
\end{array}\right)
$$

the Dirac conjugation $\bar{\chi}^{\alpha}=\chi^{\dagger \dot{\alpha}} A_{\dot{\alpha}}{ }^{\alpha}$ depends on the matrix $A$, which obeys

$$
A \gamma^{a} A^{-1}=\left(\gamma^{a}\right)^{\dagger}, \quad A^{\dagger}=A
$$

We make the usual choice

$$
A=\left(\begin{array}{cc}
0 & 1 \\
1 & 0
\end{array}\right)=\gamma^{0}
$$

The charge conjugation of a spinor using complex conjugation is

$$
\begin{gathered}
\chi^{c}=B \chi^{*} \\
B^{-1} \gamma^{a} B=-\left(\gamma^{a}\right)^{*}, \quad B B^{*}=\mathbb{1} .
\end{gathered}
$$

For our choice of $\gamma^{a}(330)$

$$
B=\left(\begin{array}{cc}
-1 & 0 \\
0 & 1
\end{array}\right)
$$

Alternatively, one can define the charge conjugated spinor with the help of the Dirac conjugation matrix (338),

$$
\chi^{c}=(\bar{\chi} C)^{T}=C^{T} A^{T} \chi^{*}, \quad \chi_{\alpha}^{c}=\bar{\chi}^{\beta} C_{\beta \alpha},
$$




$$
\begin{gathered}
C^{-1} \gamma^{a} C=-\left(\gamma^{a}\right)^{T}, \quad C^{T}=-C, \\
C=\left(C_{\beta \alpha}\right)=\left(\begin{array}{cc}
0 & 1 \\
-1 & 0
\end{array}\right) .
\end{gathered}
$$

By means of

$$
\epsilon_{\alpha \beta}=\epsilon^{\alpha \beta}=\left(\begin{array}{cc}
0 & 1 \\
-1 & 0
\end{array}\right)
$$

indices of Majorana spinors $\chi^{c}=\chi$, in components $\chi_{+}=-\left(\chi_{+}\right)^{*}, \chi_{-}=\left(\chi_{-}\right)^{*}$, can be raised and lowered as $\chi^{\alpha}=\epsilon^{\alpha \beta} \chi_{\beta}$ and $\chi_{\alpha}=\chi^{\beta} \epsilon_{\beta \alpha}$. In components we get

$$
\chi^{+}=\chi_{-}, \quad \chi^{-}=-\chi_{+} .
$$

This yields $\varphi^{\alpha} \chi_{\alpha}=-\varphi_{\alpha} \chi^{\alpha}=\chi^{\alpha} \varphi_{\alpha}=\varphi^{-} \chi^{+}-\varphi^{+} \chi^{-}$for two anticommuting Majorana spinors $\varphi$ and $\chi$. For bilinear forms we use the shorthand

$$
(\varphi \chi)=\varphi^{\alpha} \chi_{\alpha}, \quad\left(\varphi \gamma^{a} \chi\right)=\varphi^{\alpha} \gamma_{\alpha}^{a} \chi_{\beta}, \quad\left(\varphi \gamma^{3} \chi\right)=\varphi^{\alpha} \gamma_{\alpha}^{3} \chi_{\beta}
$$

A useful property is

$$
\epsilon_{\alpha \beta} \epsilon^{\gamma \delta}=\delta_{\alpha}^{\gamma} \delta_{\beta}^{\delta}-\delta_{\alpha}^{\delta} \delta_{\beta}^{\gamma}
$$

The Fierz identity (334) yields

$$
\chi_{\alpha} \varphi^{\beta}=-\frac{1}{2}(\varphi \chi) \delta_{\alpha}{ }^{\beta}-\frac{1}{2}\left(\varphi \gamma_{a} \chi\right) \gamma_{\alpha}^{a}{ }^{\beta}-\frac{1}{2}\left(\varphi \gamma^{3} \chi\right) \gamma_{\alpha}^{3}{ }^{\beta}
$$

Among the spinor matrices $\left(\gamma^{a}\right)^{\alpha \beta}$ and $\left(\gamma^{3}\right)^{\alpha \beta}$ are symmetric in $\alpha \leftrightarrow \beta$, whereas $\epsilon^{\alpha \beta}$ is antisymmetric.

The chiral projectors

$$
P_{ \pm}=\frac{1}{2}\left(\mathbb{1} \pm \gamma^{3}\right)
$$

project $\chi_{+}$and $\chi_{-}$.

Light cone components of a vector $v^{a}$ are defined by

$$
\left(\begin{array}{c}
v^{++} \\
v^{--}
\end{array}\right)=\frac{i}{\sqrt{2}}\left(\begin{array}{r}
v^{0}+v^{1} \\
-v^{0}+v^{1}
\end{array}\right) .
$$

Then the indices $(++,--)$ exactly coincide with components of the 2 -spinor $v^{\alpha \beta}$ which can be related to a Lorentz vector $v^{a}$ by

$$
v^{\alpha \beta}:=\frac{i}{\sqrt{2}} v^{a} \gamma_{a}^{\alpha \beta}
$$


where $\gamma^{a}$ is given by (330). The pre-factor (up to a sign) is necessary for (352) to be consistent with the metric in light-cone coordinates $\eta_{++--}=\eta_{--++}=1$. This metric can be extended to a full metric in 2-spinor space

$$
\eta_{\alpha \beta}{ }^{\delta \gamma}=-\frac{1}{2} \gamma_{\alpha \beta}^{a} \gamma_{a}{ }^{\delta \gamma}-\frac{1}{2} \gamma_{\alpha \beta}^{3} \gamma^{3 \delta \gamma}-\frac{1}{2} \epsilon_{\alpha \beta} \epsilon^{\delta \gamma},
$$

where the Fierz identity (334) yields

$$
\eta_{\alpha \beta}^{\delta \gamma}=\delta_{\alpha}^{\gamma} \delta_{\beta}^{\delta} .
$$

\section{References}

[1] P. Jordan, Schwerkraft und Weltall. Braunschweig, second ed., 1955.

[2] R. H. Dicke Rev. Mod. Phys. 29 (1957) 29.

[3] P. Jordan, "The present state of Dirac's cosmological hypothesis," Z. Phys. 157 (1959) 112-121.

[4] M. Fierz, "Über die physikalische Deutung der erweiterten Gravitationstheorie P. Jordans," Helv. Phys. Acta 29 (1956) 128.

[5] C. Brans and R. H. Dicke, "Mach's principle and a relativistic theory of gravitation," Phys. Rev. 124 (1961) 925-935.

[6] L. min Wang, R. R. Caldwell, J. P. Ostriker, and P. J. Steinhardt, "Cosmic concordance and quintessence," Astrophys. J. 530 (2000) 17-35, astro-ph/9901388.

[7] L. M. Diaz-Rivera and L. O. Pimentel, "Cosmological models with dynamical $\Lambda$ in scalar-tensor theories," Phys. Rev. D60 (1999) 123501, gr-qc/9907016.

[8] T. Matos, F. S. Guzman, and L. A. Urena-Lopez, "Scalar field as dark matter in the universe," Class. Quant. Grav. 17 (2000) 1707, astro-ph/9908152.

[9] A. A. Coley, "Qualitative properties of scalar-tensor theories of gravity," Gen. Rel. Grav. 31 (1999) 1295, astro-ph/9910395.

[10] O. Bertolami and P. J. Martins, "Non-minimal coupling and quintessence," Phys. Rev. D61 (2000) 064007, gr-qc/9910056.

[11] L. M. Sokolowski, "Universality of Einstein's general relativity," gr-qc/9511073. 
[12] The Supernova Cosmology Project Collaboration, S. Perlmutter et al., "Cosmology from type Ia supernovae," Bull. Am. Astron. Soc. 29 (1997) 1351, astro-ph/9812473.

[13] Supernova Cosmology Project Collaboration, S. Perlmutter et al., "Measurements of $\Omega$ and $\Lambda$ from 42 high-redshift supernovae," astro-ph/9812133.

[14] A. G. Riess et al., "Observational evidence from supernovae for an accelerating universe and a cosmological constant," Astron. J. 116 (1998) 1009-1038, astro-ph/9805201.

[15] P. M. Garnavich et al., "Constraints on cosmological models from hubble space telescope observations of high-z supernovae," Astrophys. J. 493 (1998) L53-57, astro-ph/9710123.

[16] P. M. Garnavich et al., "Supernova limits on the cosmic equation of state," Astrophys. J. 509 (1998) 74, astro-ph/9806396.

[17] B. Schmidt et al., "The high-z supernova search," Astrophys. J. 507 (1998) 46, astro-ph/9805200.

[18] K. Hayashi, "The gauge theory of the translation group and underlying geometry," Phys. Lett. B69 (1977) 441.

[19] K. Hayashi and T. Shirafuji, "New general relativity," Phys. Rev. D19 (1979) 3524-3553. Addendum-ibid. D24 (1981) 3312-3314.

[20] F. W. Hehl, "Four lectures on Poincare gauge field theory," in Proceedings of the 6th School of Cosmology and Gravitation on Spin, Torsion, Rotation and Supergravity, P. G. Bergmann and V. de Sabbata, eds., vol. 58 of Series B: Physics. NATO Advanced Study Institute, Erice, May 6-18 1979, 1980.

[21] W. Kopczyński, "Problems with metric-teleparallel theories of gravitation," J. Phys. A15 (1982) 493-506.

[22] W. Kopczyński, "Variational principles for gravity and fluids," Annals Phys. 203 (1990) 308.

[23] F. Müller-Hoissen and J. Nitsch, "Teleparallelism - a viable theory of gravity?," Phys. Rev. D28 (1983) 718.

[24] F. Müller-Hoissen and J. Nitsch, "On the tetrad theory of gravity," Gen. Rel. Grav. 17 (1985) 747-760. 
[25] P. Baekler and E. W. Mielke, "Hamiltonian structure of Poincare gauge theory and separation of nondynamical variables in exact torsion solutions," Fortschr. Phys. 36 (1988) 549.

[26] E. W. Mielke, "Positive gravitational energy proof from complex variables?," Phys. Rev. D42 (1990) 3388-3394.

[27] E. W. Mielke, "Ashtekar's complex variables in general relativity and its teleparallelism equivalent," Ann. Phys. 219 (1992) 78.

[28] J. M. Nester, "Positive energy via the teleparallel Hamiltonian," Int. J. Mod. Phys. A4 (1989) 1755-1772.

[29] F. W. Hehl, J. D. McCrea, E. W. Mielke, and Y. Neeman, "Metric affine gauge theory of gravity: Field equations, Noether identities, world spinors, and breaking of dilation invariance," Phys. Rept. 258 (1995) 1-171, gr-qc/9402012.

[30] D. Z. Freedman, P. van Nieuwenhuizen, and S. Ferrara, "Progress toward a theory of supergravity," Phys. Rev. D13 (1976) 3214-3218.

[31] D. Z. Freedman and P. van Nieuwenhuizen, "Properties of supergravity theory," Phys. Rev. D14 (1976) 912.

[32] S. Deser and B. Zumino, "Consistent supergravity," Phys. Lett. B62 (1976) 335.

[33] S. Deser and B. Zumino, "A complete action for the spinning string," Phys. Lett. B65 (1976) 369-373.

[34] M. B. Green, J. H. Schwarz, and E. Witten, Superstring Theory, vol. 1 and 2. Cambridge University Press, Cambridge, 1987.

[35] D. Lüst and S. Theisen, Lectures on String Theory. Springer-Verlag, Berlin, 1989.

[36] J. Polchinski, String Theory, vol. I and II. Cambridge University Press, Cambridge, 1998.

[37] H. J. Schmidt, "The classical solutions of two-dimensional gravity," Gen. Rel. Grav. 31 (1999) 1187, gr-qc/9905051.

[38] Y. N. Obukhov and F. W. Hehl, "Black holes in two dimensions," in Black Holes: Theory and Observation, F. W. Hehl, C. Kiefer, and R. J. Metzler, eds., pp. 289-316. Springer 1998, Proceedings of the 179. WE-Heraeus Seminar in Bad Honnef, Germany, 1997. hep-th/9807101. 
[39] T. Strobl, Gravity in Two Spacetime Dimensions. Habilitationsschrift, Rheinisch-Westfälische Technische Hochschule Aachen, 1999.

[40] M. O. Katanaev and I. V. Volovich, "String model with dynamical geometry and torsion," Phys. Lett. B175 (1986) 413-416.

[41] M. O. Katanaev and I. V. Volovich, "Two-dimensional gravity with dynamical torsion and strings," Ann. Phys. 197 (1990) 1.

[42] P. Schaller and T. Strobl, "Canonical quantization of non-Einsteinian gravity and the problem of time," Class. Quant. Grav. 11 (1994) 331-346, hep-th/9211054.

[43] P. Schaller and T. Strobl, "Poisson structure induced (topological) field theories," Mod. Phys. Lett. A9 (1994) 3129-3136, hep-th/9405110.

[44] T. Banks and M. O'Loughlin, "Two-dimensional quantum gravity in Minkowski space," Nucl. Phys. B362 (1991) 649-664.

[45] S. D. Odintsov and I. L. Shapiro, "One loop renormalization of two-dimensional induced quantum gravity," Phys. Lett. B263 (1991) $183-189$.

[46] D. Louis-Martinez, J. Gegenberg, and G. Kunstatter, "Exact Dirac quantization of all 2-d dilaton gravity theories," Phys. Lett. B321 (1994) 193-198, gr-qc/9309018.

[47] J. Gegenberg, G. Kunstatter, and D. Louis-Martinez, "Observables for two-dimensional black holes," Phys. Rev. D51 (1995) 1781-1786, gr-qc/9408015.

[48] T. Klösch and T. Strobl, "Classical and quantum gravity in (1+1)-dimensions. Part 1: A unifying approach," Class. Quant. Grav. 13 (1996) 965-984, gr-qc/9508020. Erratum ibid. 14 (1997) 825.

[49] T. Klösch and T. Strobl, "Classical and quantum gravity in 1+1 dimensions. Part 2: The universal coverings," Class. Quant. Grav. 13 (1996) 2395-2422, gr-qc/9511081.

[50] T. Klösch and T. Strobl, "Classical and quantum gravity in $(1+1)$-dimensions. Part 3: Solutions of arbitrary topology," Class. Quant. Grav. 14 (1997) 1689-1723.

[51] T. Klosch and T. Strobl, "A global view of kinks in 1+1 gravity," Phys. Rev. D57 (1998) 1034-1044, gr-qc/9707053. 
[52] M. O. Katanaev, W. Kummer, and H. Liebl, "Geometric interpretation and classification of global solutions in generalized dilaton gravity," Phys. Rev. D53 (1996) 5609-5618, gr-qc/9511009.

[53] M. O. Katanaev, W. Kummer, and H. Liebl, "On the completeness of the black hole singularity in 2d dilaton theories," Nucl. Phys. B486 (1997) 353-370, gr-qc/9602040.

[54] H. Verlinde, "Black holes and strings in two dimensions," in The Sixth Marcel Grossmann Meeting on General Relativity, Kyoto, Japan 1991, H. Sato, ed., pp. 813-831. World Scientific, Singapore, 1992.

[55] W. Kummer, H. Liebl, and D. V. Vassilevich, "Exact path integral quantization of generic 2-d dilaton gravity," Nucl. Phys. B493 (1997) 491-502, gr-qc/9612012.

[56] W. Kummer, H. Liebl, and D. V. Vassilevich, "Non-perturbative path integral of $2 \mathrm{~d}$ dilaton gravity and two-loop effects from scalar matter," Nucl. Phys. B513 (1998) 723, hep-th/9707115.

[57] W. Kummer, H. Liebl, and D. V. Vassilevich, "Integrating geometry in general 2d dilaton gravity with matter," Nucl. Phys. B544 (1999) 403, hep-th/9809168.

[58] P. Thomi, B. Isaak, and P. Hajicek, "Spherically symmetric systems of fields and black holes. 1. definition and properties of apparent horizon," Phys. Rev. D30 (1984) 1168.

[59] P. Hajicek, "Spherically symmetric systems of fields and black holes. 2. apparent horizon in canonical formalism," Phys. Rev. D30 (1984) 1178.

[60] H. J. Schmidt, "A new proof of Birkhoff's theorem," Grav. Cosmol. 3 (1997) 185, gr-qc/9709071.

[61] H. J. Schmidt, "A two-dimensional representation of four-dimensional gravitational waves," Int. J. Mod. Phys. D7 (1998) 215-224, gr-qc/9712034.

[62] B. M. Barbashov, V. V. Nesterenko, and A. M. Chervyakov, "The solitons in some geometrical field theories," Theor. Math. Phys. 40 (1979) 572-581. Teor. Mat. Fiz. 40 (1979) 15-27, J. Phys. A13 (1980) 301-312.

[63] C. Teitelboim, "Gravitation and hamiltonian structure in two space-time dimensions," Phys. Lett. B126 (1983) 41. 
[64] C. Teitelboim, "The Hamiltonian structure of two-dimensional space-time and its relation with the conformal anomaly," in Quantum Theory of Gravity. Essays in Honor of the 60th Birthday of Bryce S. DeWitt, S. Christensen, ed., pp. 327-344. Hilger, Bristol, 1984.

[65] R. Jackiw, "Liouville field theory: a two-dimensional model for gravity," in Quantum Theory of Gravity. Essays in Honor of the 60th Birthday of Bryce S. DeWitt, S. Christensen, ed., pp. 403-420. Hilger, Bristol, 1984.

[66] R. Jackiw, "Lower dimensional gravity," Nucl. Phys. B252 (1985) 343-356.

[67] G. Mandal, A. M. Sengupta, and S. R. Wadia, "Classical solutions of two-dimensional string theory," Mod. Phys. Lett. A6 (1991) 1685-1692.

[68] S. Elitzur, A. Forge, and E. Rabinovici, "Some global aspects of string compactifications," Nucl. Phys. B359 (1991) 581-610.

[69] E. Witten, "On string theory and black holes," Phys. Rev. D44 (1991) $314-324$.

[70] C. G. Callan Jr., S. B. Giddings, J. A. Harvey, and A. Strominger, "Evanescent black holes," Phys. Rev. D45 (1992) 1005-1009, hep-th/9111056.

[71] A. Miković, "Exactly solvable models of 2-d dilaton quantum gravity," Phys. Lett. B291 (1992) 19-25, hep-th/9207006.

[72] A. Miković, "Two-dimensional dilaton gravity in a unitary gauge," Phys. Lett. B304 (1993) 70-76, hep-th/9211082.

[73] A. Miković, "Hawking radiation and back reaction in a unitary theory of 2-d quantum gravity," Phys. Lett. B355 (1995) 85-91, hep-th/9407104.

[74] A. Miković and V. Radovanović, "Loop corrections in the spectrum of 2d Hawking radiation," Class. Quant. Grav. 14 (1997) 2647-2661, gr-qc/9703035.

[75] K. V. Kuchař, J. D. Romano, and M. Varadarajan, "Dirac constraint quantization of a dilatonic model of gravitational collapse," Phys. Rev. D55 (1997) 795-808, gr-qc/9608011.

[76] M. Varadarajan, "Quantum gravity effects in the CGHS model of collapse to a black hole," Phys. Rev. D57 (1998) 3463-3473, gr-qc/9801058.

[77] D. Cangemi, R. Jackiw, and B. Zwiebach, "Physical states in matter coupled dilaton gravity," Ann. Phys. 245 (1996) 408-444, hep-th/9505161. 
[78] E. Benedict, R. Jackiw, and H. J. Lee, "Functional Schrödinger and BRST quantization of (1+1)-dimensional gravity," Phys. Rev. D54 (1996) 6213-6225, hep-th/9607062.

[79] P. Schaller and T. Strobl, "Poisson sigma models: A generalization of 2d gravity Yang-Mills systems," hep-th/9411163.

[80] P. Schaller and T. Strobl, "Introduction to Poisson- $\sigma$ models," in Low-Dimensional Models in Statistical Physics and Quantum Field Theory, H. Grosse and L. Pittner, eds., vol. 469 of Lecture Notes in Physics, p. 321. Springer, Berlin, 1996. hep-th/9507020.

[81] N. Ikeda and K. I. Izawa, "Quantum gravity with dynamical torsion in two-dimensions," Prog. Theor. Phys. 89 (1993) 223-230.

[82] N. Ikeda and K. I. Izawa, "Gauge theory based on quadratic Lie algebras and 2-d gravity with dynamical torsion," Prog. Theor. Phys. 89 (1993) $1077-1086$.

[83] N. Ikeda and K. I. Izawa, "General form of dilaton gravity and nonlinear gauge theory," Prog. Theor. Phys. 90 (1993) 237-246, hep-th/9304012.

[84] A. S. Cattaneo and G. Felder, "A path integral approach to the Kontsevich quantization formula," Commun. Math. Phys. 212 (2000) 591, math.qa/9902090.

[85] A. S. Cattaneo and G. Felder, "Poisson sigma models and symplectic groupoids," math.sg/0003023.

[86] T. Strobl, Poisson Structure Induced Field Thoeries and Models of $1+1$ Dimensional Gravity. PhD thesis, Vienna University of Technology, May, 1994.

[87] A. Weinstein, "The local structure of Poisson manifolds," J. Diff. Geom. 18 (1983) 523-557.

[88] Y. Choquet-Bruhat and C. DeWitt-Morette, Analysis, Manifolds and Physics. Part II: 92 Applications. North-Holland, Amsterdam, 1989.

[89] W. Kummer and P. Widerin, "Conserved quasilocal quantities and general covariant theories in two-dimensions," Phys. Rev. D52 (1995) 6965-6975, gr-qc/9502031.

[90] D. Grumiller and W. Kummer, "Absolute conservation law for black holes," Phys. Rev. D61 (2000) 064006, gr-qc/9902074. 
[91] F. A. Berezin, The Method of Second Quantization. Academic Press, New York and London, 1966.

[92] B. DeWitt, Supermanifolds. Cambridge Monographs on Mathematical Physics. Cambridge University Press, 1984.

[93] V. S. Vladimirov and I. V. Volovich, "Superanalysis. I. Differential calculus," Theor. Math. Phys. 59 (1984) 317-335.

[94] V. S. Vladimirov and I. V. Volovich, "Superanalysis. II. Integral calculus," Theor. Math. Phys. 60 (1985) 743-765.

[95] F. Constantinescu and H. F. de Groote, Geometrische und algebraische Methoden der Physik: Supermannigfaltigkeiten und Virasoro-Algebren. Teubner Studienbücher Mathematik. Teubner, Stuttgart, 1994.

[96] T. Strobl, "Comment on gravity and the Poincaré group," Phys. Rev. D48 (1993) 5029-5031, hep-th/9302041.

[97] P. van Nieuwenhuizen, "Supergravity," Phys. Rept. 68 (1981) 189-398.

[98] P. S. Howe, "Super Weyl transformations in two dimensions," J. Phys. A12 (1979) 393-402.

[99] Y. Park and A. Strominger, "Supersymmetry and positive energy in classical and quantum two-dimensional dilaton gravity," Phys. Rev. D47 (1993) 1569-1575, hep-th/9210017.

[100] A. H. Chamseddine, "Superstrings in arbitrary dimensions," Phys. Lett. B258 (1991) 97-103.

[101] M. F. Ertl, M. O. Katanaev, and W. Kummer, "Generalized supergravity in two dimensions," Nucl. Phys. B530 (1998) 457-486, hep-th/9710051.

[102] V. O. Rivelles, "Topological two-dimensional dilaton supergravity," Phys. Lett. B321 (1994) 189-192, hep-th/9301029.

[103] D. Cangemi and M. Leblanc, "Two-dimensional gauge theoretic supergravities," Nucl. Phys. B420 (1994) 363-378, hep-th/9307160.

[104] N. Ikeda, "Gauge theory based on nonlinear Lie superalgebras and structure of 2-d dilaton supergravity," Int. J. Mod. Phys. A9 (1994) $1137-1152$.

[105] N. Ikeda, "Two-dimensional gravity and nonlinear gauge theory," Ann. Phys. 235 (1994) 435-464, hep-th/9312059. 
[106] J. M. Izquierdo, "Free differential algebras and generic 2d dilatonic (super)gravities," Phys. Rev. D59 (1999) 084017, hep-th/9807007.

[107] T. Strobl, "Target-superspace in 2d dilatonic supergravity," Phys. Lett. B460 (1999) 87, hep-th/9906230.

[108] V. G. Kac, "A sketch of Lie superalgebra theory," Commun. Math. Phys. 53 (1977) 31-64.

[109] M. Scheunert, W. Nahm, and V. Rittenberg, "Classification of all simple graded Lie algebras whose Lie algebra is reductive. 1," J. Math. Phys. 17 (1976) 1626.

[110] M. Scheunert, W. Nahm, and V. Rittenberg, "Classification of all simple graded Lie algebras whose Lie algebra is reductive. 2. (construction of the exceptional algebras)," J. Math. Phys. 17 (1976) 1640.

[111] L. Frappat, P. Sorba, and A. Sciarrino, "Dictionary on Lie superalgebras," hep-th/9607161.

[112] J. de Boer, F. Harmsze, and T. Tjin, "Nonlinear finite $W$-symmetries and applications in elementary systems," Phys. Rept. 272 (1996) 139-214, hep-th/9503161.

[113] L. Brink and J. H. Schwarz, "Local complex supersymmetry in two-dimensions," Nucl. Phys. B121 (1977) 285.

[114] M. Ertl, "Mathematica Index Package." A Mathematica Package for index manipulation. Endowed with anticommutative product as well as left and right derivatives suitable for super calculation. Version 0.14.2. Unpublished.

[115] D. Cangemi and R. Jackiw, "Gauge invariant formulations of lineal gravity," Phys. Rev. Lett. 69 (1992) 233-236, hep-th/9203056.

[116] D. Cangemi and R. Jackiw, "Poincare gauge theory for gravitational forces in (1+1)- dimensions," Ann. Phys. 225 (1993) 229-263, hep-th/9302026.

[117] A. Y. Alekseev, P. Schaller, and T. Strobl, "The topological $G / G$ WZW model in the generalized momentum representation," Phys. Rev. D52 (1995) 7146-7160, hep-th/9505012.

[118] M. Ertl, "PhD thesis; in preparation." Vienna University of Technology. 